REVIEWS OF MODERN PHYSICS, VOLUME 84, APRIL-JUNE 2012

\title{
Ice structures, patterns, and processes: A view across the icefields
}

Thorsten Bartels-Rausch

Laboratory of Radiochemistry and Environmental Chemistry, Paul Scherrer Institut, CH-5232 Villigen PSI, Switzerland

Vance Bergeron

Ecole Normale Supérieure de Lyon, F-69007 Lyon, France

Julyan H. E. Cartwright

Instituto Andaluz de Ciencias de la Tierra, CSIC_Universidad de Granada,

E-18071 Granada, Spain

Rafael Escribano

Instituto de Estructura de la Materia, CSIC, E-28006 Madrid, Spain

John L. Finney

Department of Physics and Astronomy, and London Centre for Nanotechnology, University College London, London WC1E 6BT, United Kingdom

Hinrich Grothe

Institute of Materials Chemistry, Vienna University of Technology,

A-1060 Vienna, Austria

Pedro J. Gutiérrez

Instituto de Astrofísica de Andalucía, CSIC, E-18080 Granada, Spain

Jari Haapala

Finnish Meteorological Institute, FIN-00100 Helsinki, Finland

Werner F. Kuhs

GZG Crystallography, University of Göttingen, D-37077 Göttingen, Germany

Jan B. C. Pettersson

Department of Chemistry, Atmospheric Science, University of Gothenburg, SE-41296 Göteborg, Sweden

Stephen D. Price

Department of Chemistry, University College London, London WC1H OAJ,

United Kingdom

C. Ignacio Sainz-Díaz

Instituto Andaluz de Ciencias de la Tierra, CSIC_Universidad de Granada, E-18002 Granada, Spain

Debbie J. Stokes

FEl Company, Achtseweg Noord 5, 5651 GG, Eindhoven, The Netherlands

Giovanni Strazzulla

INAF-Osservatorio Astrofisico di Catania, I-95123 Catania, Italy

Erik S. Thomson

Department of Chemistry, Atmospheric Science, University of Gothenburg, SE-41296 Göteborg, Sweden 


\author{
Hauke Trinks \\ Technical University Hamburg Harburg, D-21079 Hamburg, Germany \\ Nevin Uras-Aytemiz \\ Department of Chemistry, Suleyman Demirel University, TR-32260 Isparta, Turkey
}

(published 24 May 2012)

\begin{abstract}
From the frontiers of research on ice dynamics in its broadest sense, this review surveys the structures of ice, the patterns or morphologies it may assume, and the physical and chemical processes in which it is involved. Open questions in the various fields of ice research in nature are highlighted, ranging from terrestrial and oceanic ice on Earth, to ice in the atmosphere, to ice on other Solar System bodies and in interstellar space.
\end{abstract}

DOI: $10.1103 /$ RevModPhys.84.885

PACS numbers: 33.15. $-\mathrm{e}, 42.68 . \mathrm{Ge}, 83.80 . \mathrm{Nb}, 92.40 . \mathrm{Vq}$

\section{CONTENTS}

I. Introduction

II. Ice Structures

A. Order and disorder in crystalline ice structures

1. Molecular structures of ices

2. Hydrogen disorder

3. Ordering the familiar ice Ih

4. Ordering high-pressure ices

5. Some outstanding questions

B. Cubic ice

C. Amorphous ices

1. Crystalline and amorphous structures

2. Formation and structures of amorphous ices

3. Some outstanding questions

D. Polycrystallinity and interfaces

1. Ice-e pluribus unum

2. Surfaces and interfaces

III. Astrophysical Ice
A. Laboratory studies of astrophysical ices
1. Ice morphology on interstellar grain surfaces
2. Mixed ices
B. Cometary ice
1. Amorphous versus crystalline ice
2. Clathrates
C. Heterogeneous chemical processes on interstellar surfaces
1. Laboratory experiments
2. Desorption of ice-trapped molecules

IV. Atmospheric Ice
A. Measurement and simulation methods
1. Experimental techniques
2. Theoretical methods
B. Ice clouds in the mesopause region
C. Polar stratospheric clouds
D. Ice-containing clouds in the troposphere
1. Ice morphology
2. Ice nucleation in the troposphere
3. Cirrus clouds and the supersaturation puzzle
4. Tropospheric chemistry

V. Terrestrial Ice
A. Glaciers
1. Glacier flow

2. Glacial ablation $\quad 915$

3. Sintering processes 915

B. Snow chemistry 916

1. Uptake of trace gases 917

2. Photochemistry of trace gases 919

VI. Sea Ice 920

A. Processes determining the evolution of pack ice 921

1. Initial formation of sea ice 922

2. Fracturing 922

3. Redistribution 923

4. Aging 923

B. A promoter of the emergence of the first life? 924

VII. Perspectives $\quad 927$

\section{INTRODUCTION}

The ice was here, the ice was there, the ice was all around.

\section{Samuel Taylor Coleridge, The Rime of the Ancient Mariner}

Ice is indeed all around us. As the cryosphere, ice or snow covers a small but significant part of the Earth's surface, both land and sea, and it plays a similarly important role in our atmosphere. Moreover ice is present on many other celestial bodies in our Solar System and surely beyond, and it coats grains of dust in interstellar space. Ice is not a static medium but a dynamical one; it shows strong variations of its characteristics with time and place, as we may readily experience at a human scale on any ski slope. A better understanding of ice structures, patterns, and processes is thus a topic of current research in physics. We show in the following how progress in understanding these questions is elemental in understanding current questions in astrophysical, atmospheric, cryospheric, and environmental science.

Ice research questions are not only tackled separately within distinct fields, in terrestrial, oceanic, atmospheric, planetary, and interstellar ice research, but also by researchers with disparate backgrounds: by modelers, field and laboratory experimentalists, and theoreticians from both physics and 
chemistry. We work in these different fields and come from a variety of backgrounds. We came together, some of us initially for a Spanish national project supported by the Spanish CSIC, and then the majority of us for a workshop, Euroice 2008, sponsored by the European Science Foundation, which was organized to connect people working on structures and those working in applied icefields, to find common ground in the physical and chemical processes at icy surfaces and the physics and chemistry of ice structures from the molecular scale to the macroscale, and to explore whether some of the questions we were asking and some of the answers we were seeking are the same.

During the workshop we found that, despite the diversity of ice research, a number of key themes are indeed common between the different fields. The common ground in any field of ice research is the urge to understand better its structure and dynamics. For example: What are the ordering mechanisms of ice as it changes from one of its phases into another? What is the structure at its surface and how does this differ from the bulk? What is the structure and microenvironment at the contact area of ice crystals? How does ice structure form initially? Are there metastable phases present in the environment? This work focuses on this common ground. It thus does not aim to be a comprehensive review; such a review of ice physics and chemistry would be a book, and indeed there are excellent books available (Hobbs, 1974; Petrenko and Whitworth, 1999). However, many of the points raised in this work are issues of the 21 st century that are not addressed in the textbooks. This article provides a view of the way ahead from some frontiers of research on ice. We set out the main open physical and chemical questions on ice structures, patterns, and processes from the fields of ice research in nature: from ice on Earth, in the oceans and the atmosphere, to planetary and interstellar ice.

We begin in Sec. II by introducing open questions in the molecular structures of ices; we then examine open issues on dynamical patterns and processes in ice. Following this we look first, in Sec. III, at astrophysical ice. We then focus on ice on Earth, beginning with Sec. IV on atmospheric ice, whose precipitation leads to the subsequent formation of terrestrial ice, Sec. V, and sea ice, Sec. VI. We conclude in Sec. VII, in which we attempt to list the important open questions on ice from the perspectives of these different fields of ice research.

\section{ICE STRUCTURES}

"Now suppose," chortled Dr. Breed, enjoying himself, "that there were many possible ways in which water could crystallize, could freeze. Suppose that the sort of ice we skate upon and put into highballs - what we might call ice-one-is only one of several types of ice. Suppose water always froze as ice-one on Earth because it had never had a seed to teach it how to form ice-two, ice-three, ice-four ...? And suppose," he rapped on his desk with his old hand again, "that there were one form, which we will call ice-nine - a crystal as hard as this deskwith a melting point of, let us say, one-hundred degrees Fahrenheit, or, better still, a melting point of one-hundred-and-thirty degrees."

Kurt Vonnegut, Cat's Cradle

\section{A. Order and disorder in crystalline ice structures}

Unlike Vonnegut's fictional ice-nine, the real ice IX is not stable at ambient pressures and temperatures. But there are indeed many phases of ice. Although we normally experience only one of these, the familiar ice Ih that forms in your freezer and makes up snowflakes and icebergs, changing pressure and temperature can cause changes of phase into other forms, as indicated in the phase diagram of Fig. 1. Most of these phases are stable within a given range of temperature and pressure, but some are only metastable; for example, ice IV and XII, also indicated in Fig. 1, are found within the regions of stability of other phases.

\section{Molecular structures of ices}

We now know the molecular structures of all of these phases, thanks largely to neutron-diffraction crystallographic studies: These are indicated by the insets in Fig. 1. Further details and references can be found in Finney (2001, 2004) and Petrenko and Whitworth (1999). These structures can be simply rationalized in terms of fully connected tetrahedral networks of water molecules, with each molecule donating hydrogen bonds to two neighbors and accepting two hydrogen bonds from two others (Fig. 2). In the low-pressure ice Ih, the $\mathrm{O}-\mathrm{O}-\mathrm{O}$ angles are close to the ideal tetrahedral angle of $109.47^{\circ}$. As pressure is increased, the molecules have to rearrange themselves to occupy less volume and this is done initially by both changes to the network structure (but still retaining four coordination) and increased distortion of the $\mathrm{O}-\mathrm{O}-\mathrm{O}$ angles: for example, in ice II these angles vary between $80^{\circ}$ and $129^{\circ}$. As we increase pressure further, we come to a point at which the reduced volume available cannot be filled by merely increasing hydrogen-bond distortion: the water molecules then form interpenetrating networks, as in ice VII which consists essentially of two interpenetrating diamond-type lattices. But each network remains four coordinated.

\section{Hydrogen disorder}

However, what was said above refers only to the networks formed by the oxygen atoms of the water molecules. When we consider the hydrogen atoms, things become a little more complicated. In fact, all the liquidus phases of ice-those which share a phase boundary with the liquid (ices Ih, III, V, VI, and VII, see Fig. 1) — are orientationally disordered. Thus, although the oxygen atoms are related to an underlying lattice, the hydrogen atoms are not. To understand this, we refer again to Fig. 2 and note that the central water molecule is in one of six possible orientations that allow it to make four hydrogen bonds with its neighbors: These six possible orientations are shown schematically in Fig. 3. The only strong chemical constraints that a particular arrangement needs to fulfill are the Bernal-Fowler rules: Each molecule must hydrogen bond to four neighbors, and there can be only 


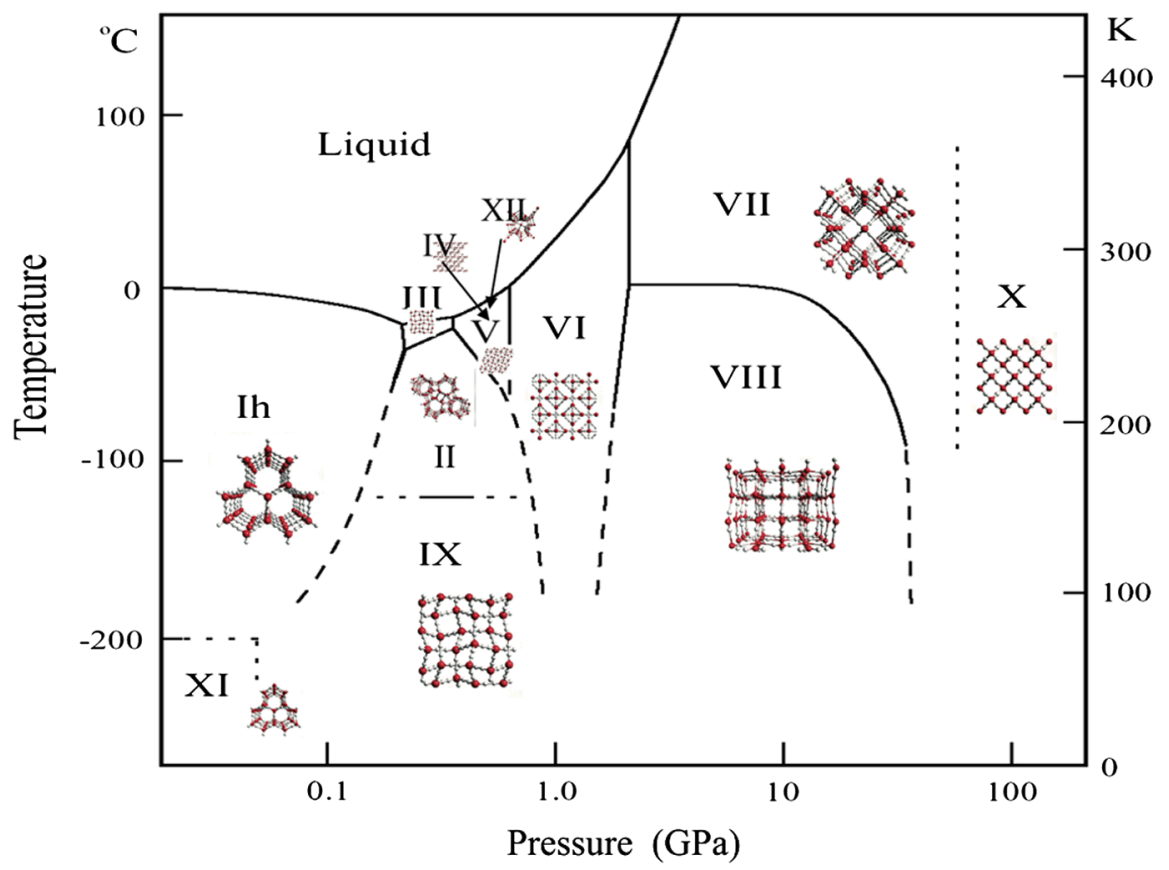

FIG. 1 (color online). The solid-liquid phase diagram of ice (the solid-liquid-gas triple point and liquid-gas coexistence line lie off the diagram to the left).

one hydrogen participating in each hydrogen bond (again, see Fig. 3). Thus, although the oxygen atoms in these ices are topologically ordered, the hydrogen atoms are not; they can be considered as decorating the underlying oxygen framework with one hydrogen along each hydrogen bond. This hydrogen disorder has another consequence that is sometimes neglected: The oxygen atoms too can show a positional disorder of several hundredths of an $\AA$ around their assigned high-symmetry crystallographic site as a response to the local hydrogen configuration (Kuhs et al., 1984; Kuhs and Lehmann, 1986). This leads in turn to local water-molecule geometries different from those obtained in a routine crystallographic structure analysis. Earlier contradictions between diffraction and spectroscopic results were removed by introducing this additional disorder. Meanwhile it has been confirmed and quantified by quantum-chemical calculations considering the very many allowed hydrogen-bond

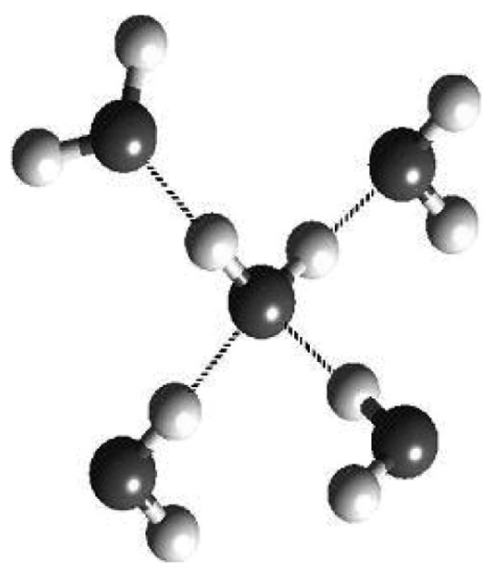

FIG. 2. The local tetrahedral coordination of water molecules in ices. Each molecule accepts two hydrogen bonds from and donates two to its neighbors. arrangements in a disordered ice phase using graph invariants (Kuo et al., 2001; Kuo and Singer, 2003); this method has been applied to ice VII-VIII (Kuo and Klein, 2004), ice Ih (Kuo, Klein, and Kuhs, 2005), ice III-IX (Knight and Singer, 2006), and ice VI (Kuo and Kuhs, 2006).

As temperature is reduced, we expect these disordered phases to order, becoming fully hydrogen ordered, and hence of lower entropy, at low temperature. This is indeed the case for ice III and ice VII, which order to ices IX and VIII, respectively (see Fig. 1). However, for all the other disordered phases, lowering the temperature does not lead to full hydrogen ordering: The molecular reorientations necessary for ordering to occur become more and more sluggish as temperature is reduced, freezing in the hydrogen, or orientational, disorder.

If we want to obtain the ordered, probably ground state, structures of the other ices, we need to find some way of unlocking the motions that enable hydrogen atoms to move in

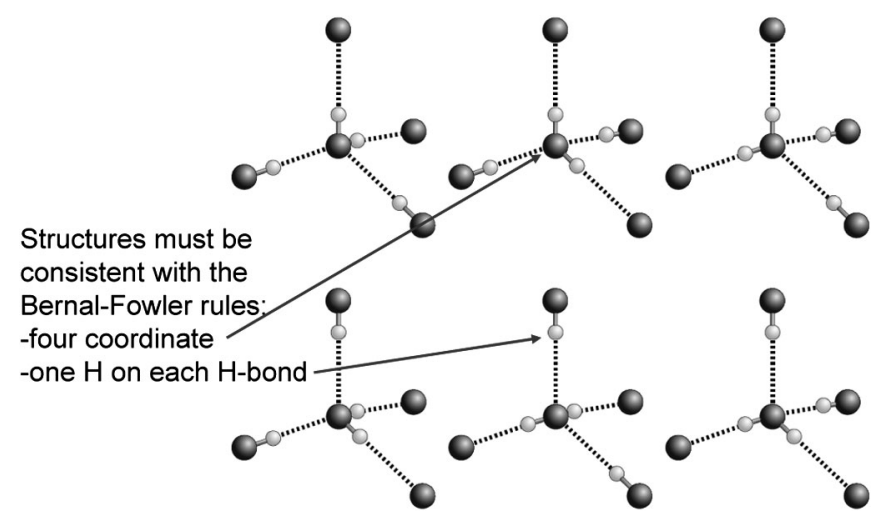

FIG. 3. Rotational hydrogen-bond disorder in ices: the six possible orientations of a central water molecule and the hydrogen bonding consequences for its four neighbors. 


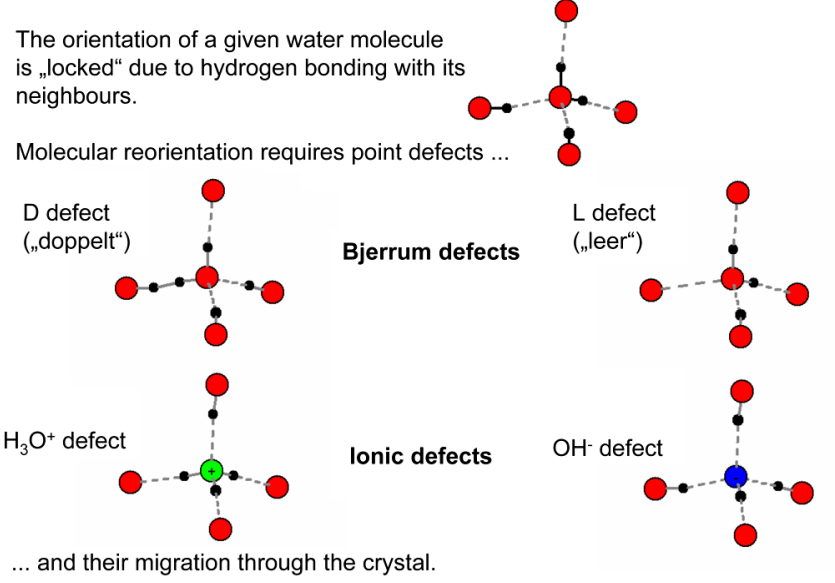

FIG. 4 (color online). Possible point defects in ice structures.

the structure, essentially to ease the ability of the molecules to rotate and hence change the hydrogen locations. In principle this might be done through inducing appropriate defects, such as so-called L and D Bjerrum defects (where the BernalFowler rules are violated by zero or double occupation of an O-O link, respectively) and ionic defects [where a water molecule is ionized to either a hydronium $\left(\mathrm{H}_{3} \mathrm{O}^{+}\right)$or hydroxyl $\left(\mathrm{OH}^{-}\right)$ion (see Fig. 4)]. In principle such defects should allow easier molecular rotations. In the case of ordering ices III and VII, presumably there is a sufficient intrinsic concentration of appropriate defects to allow ordering. In the cases of the other disordered ices that do not order on cooling, presumably the intrinsic defects are either insufficient in number or inoperative for some other reason.

\section{Ordering the familiar ice Ih}

Much effort was spent two decades ago to try to order the familiar ice Ih by forcing extrinsic defects into the structure. This was done successfully by doping with $\mathrm{KOH}$, which presumably created $\mathrm{L}$ Bjerrum and $\mathrm{OH}^{-}$defects. This doping was enough to allow careful, slow cooling partially to order the hydrogen atoms so that the ideal structure of the ordered phase (ice XI) could be determined (Jackson et al., 1997). The phase transition between the disordered (ice Ih) and ordered (ice XI) phases at $72 \mathrm{~K}\left(76 \mathrm{~K}\right.$ for $\left.\mathrm{D}_{2} \mathrm{O}\right)$ was reported first by Kawada (1972), with more precise calorimetric measurements following (Tajima, Matsuo, and Suga, 1984; Matsuo, Tajima, and Suga, 1986) in which the doped ice was held a few degrees below the transition temperature for several days to allow the growth of the ordered material. Subsequently the ideal orthorhombic structure of the ordered phase (ice XI) was determined (Jackson et al., 1997).

More recent time-resolved powder neutron-diffraction studies explored the nucleation and growth of ice XI. Working with KOD-doped deuterated ice Ih, Fukazawa et al. (2005) concluded that ice XI appears to nucleate at a temperature $57<T \leq 62 \mathrm{~K}$, some 15 to $20 \mathrm{~K}$ lower than the transition temperature. Annealing the material at $68 \mathrm{~K}$ for three days encouraged growth of the ordered phase, but the fraction of ice XI achieved leveled off at about 6\%. Further work (Fukazawa et al., 2006) exploring annealing at higher temperatures to just below the transition temperature to ice Ih doubled the fraction of the ordered phase obtained to around $12 \%$. In addition, noting that the ice XI growth rate is accelerated if the ice is annealed at temperatures lower than $64 \mathrm{~K}$ for sufficient time in advance to produce ice XI, they concluded that the thermal history of the sample influences the growth mechanisms of ice XI. A recent paper from the same group (Arakawa, Kagi, and Fukazawa, 2010) takes this work further by taking annealed, and hence ice XI containing, samples through the transition at 76 to $100 \mathrm{~K}$ to transform the sample to the disordered ice Ih. Reannealing this sample doubled the fraction of the ordered phase that was obtained. They suggest these interesting observations might be explained in terms of small hydrogen-ordered domains remaining in ice Ih, even when the ice temperature has been taken well above (here $24 \mathrm{~K}$ above) the ice Ih-XI transition temperature. This suggestion, that residual order may remain above the transition temperature, is an interesting one that may also be relevant to the ordering of other ice phases.

\section{Ordering high-pressure ices}

Early work on trying to order ice V by Handa, Klug, and Whalley (1987) observed that an endothermic peak on heating ice $\mathrm{V}$ could be intensified by $\mathrm{KOH}$ doping. This transition was discussed, therefore, in terms of a hydrogen disorderorder transition. However, hydrogen ordering could not be confirmed by Raman spectroscopy (Minčeva-Šukarova, Slark, and Sherman, 1988). Attempts at ordering the remaining phases thus remained frozen, similar to the molecular rotations themselves, for nearly 20 years.

The unfreezing of this problem occurred recently when the idea of trying acid rather than alkali doping was suggested by Salzmann and tested, initially, on ice V. This structure was thought to be a good candidate for ordering studies since Erwin Mayer in Innsbruck noted that up to 50\% ordering had been obtained in this structure by Lobban, Finney, and Kuhs (2000) without any doping. Accordingly, studies were initiated using both $\mathrm{HF}$ and $\mathrm{HCl}$ doping, coupled with carefully controlled cooling procedures. The initial Raman data taken on these preparations suggested significant ordering had been achieved, an ordering that appeared to be much greater for the HCl-doped sample than the HF-doped one. So neutron powder diffraction measurements were made (on deuterated

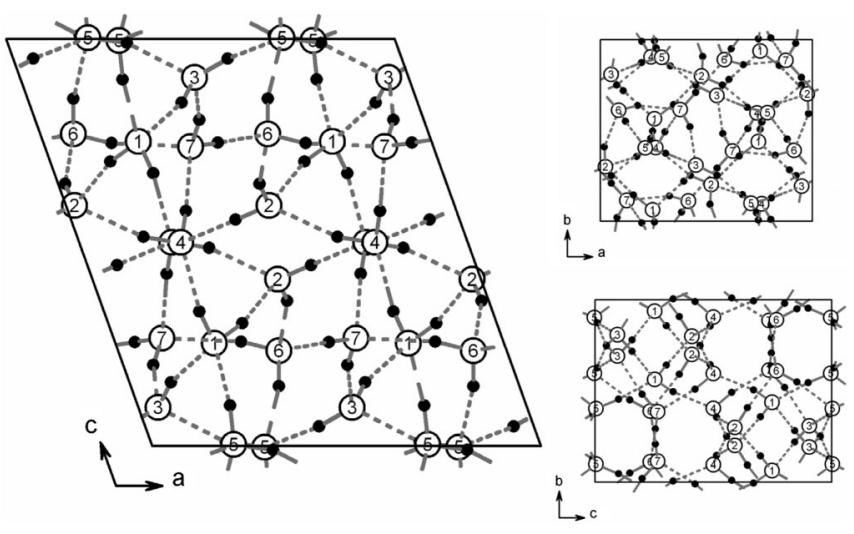

FIG. 5. The three unit cell projections of the structure of ordered ice XIII; with $28 \mathrm{H}_{2} \mathrm{O}$ molecules per unit cell it has the most complicated structure of all crystalline phases of ice. 
systems as neutrons see deuterium atoms much better than they do hydrogen atoms), and significant ordering was confirmed for DCl-doped samples. Not only was the ordered structure, named ice XIII according to the convention that has developed in the icefield, determined (see Fig. 5) (Salzmann et al., 2006), but repeated thermal cycling showed that the ice V to ice XIII order-disorder transition is reversible. Interestingly, this transition occurs with no detectable volume change, although there are clear changes in the individual crystallographic unit cell parameters.

Encouraged by this success, acid doping studies of disordered ice XII (a metastable phase) and ice VI were tried, resulting, respectively, in the new ordered phases XIV (Salzmann et al., 2006) and XV (Salzmann et al., 2009). Both of these order-disorder phase transitions are also apparently reversible. So we now know the ordered structures of all the known disordered ices apart from that of (metastable) ice IV.

\section{Some outstanding questions}

So what are the outstanding questions to be answered about the ice phase diagram, in particular, in the context of hydrogen ordering? We suggest the following to be of particular interest.

First, can we order the remaining disordered phase, ice IV and what is its structure? Being a metastable phase, this is a particularly difficult structure to make reliably. However, recently a reproducible procedure for its preparation has been found (Salzmann et al., 2002) which should facilitate future studies of its ordering.

Second, ice II is alone of all the known ice phases in occurring only as a hydrogen-ordered structure. Why is this? Can a disordered form be found, and if so what is its structure? If ice II cannot be disordered, why not? Can this be explained in simple energetic terms, with the ordered structure having the minimum free energy at this temperature, and if so, what is it about the actual structure that means that this is the case whereas ices Ih, V, and VI at similar temperatures are all disordered?

Third, can we complete the phase diagram? This requires the resolution of the structure of ordered ice IV, and also probably the discovery of at least one other phase whose presence has been seen fleetingly in neutron crystallographic survey work; for example, the unidentified "yellow phase" found by Lobban (1998) at about $0.55 \mathrm{GPa}$ (5.5 kbar) and $263 \mathrm{~K}$, a $(p, T)$ point close to where ices IV and $\mathrm{V}$ can also be found.

Fourth, can we predict the topology of the phase diagram, either by computer simulation or from first-principles quantum-chemistry calculations? For if we could, it would raise confidence in the potential functions used in simulations of aqueous systems. Recent work by Vega et al. (2009) showed that some commonly used potentials are much better than others in reproducing the predominant features of the phase diagram up to medium pressures, although their work has not as yet considered the problems of the order-disorder transitions. Work by others has, however, attempted to predict the structures of ordered ices using density-functional theory approaches, a particularly impressive example being a correct blind prediction of the structure of ice XIV (Tribello, Slater, and Salzmann, 2006). Further successes in predicting the ordered ground-state structures would increase confidence in our understanding of the water-water interaction.

Fifth, what are the ordering mechanisms for both acid (e.g., $\mathrm{HCl}$ for ices $\mathrm{V}, \mathrm{VI}$, and $\mathrm{XII}$ ) and base (e.g., $\mathrm{KOH}$ for ice $\mathrm{Ih}$ ) doping, and why is, for example, $\mathrm{HCl}$ much more effective than HF? And how might the ordering mechanism relate to the topology of the structure? Why, for example, does acid doping not work for ice Ih, whereas base doping does?

And finally, can we understand the existence of the metastable phases and their ranges of metastability? And can we perhaps use the metastable phases to try to improve our understanding of nucleation and growth mechanisms in ice? Interestingly, ice XII was first observed within the range of stability of ice V, although later work found that it could also be obtained at much lower temperatures by crystallizing from high-density amorphous ice (Koza et al., 1999, 2000; Kohl, Mayer, and Hallbrucker, 2001). Is there perhaps a region of the phase diagram where ice XII is the stable phase? The ice-V region is a particularly intriguing one in that two metastable phases, ices IV and XII, can also be found in this region. Moreover, all three phases have been observed at the same $(p, T)$ point (Lobban, 1998). Furthermore, the free-energy difference between ice V and ice XII has been experimentally estimated to be about $67 \mathrm{~J} \mathrm{~mol}^{-1}$, a very small difference that is much less than the abilities of computer simulations and ab initio approaches to estimate (Lobban, 1998). Last, in this region, as part of studies to map the stability-metastability ranges of the structures found in the ice- $\mathrm{V}$ region, it was possible to observe a reversible transition between ice IV and ice XII (J. L. Finney and F. Gotthardt, unpublished observation 2000), i.e., between two metastable phases.

Strange indeed.

\section{B. Cubic ice}

Cubic ice, ice Ic, is a metastable hydrogen-disordered ambient-pressure phase of ice. Its ubiquitous occurrence, its importance for atmospheric science (see Sec. IV) and for astrophysical issues (see Sec. III) merit a separate entry. Moreover, it turns out to be poorly crystallized and in this respect justifies a place between the crystalline and amorphous ices, possibly, as we shall see, even in a literal sense. It can be formed from a multitude of starting phases and environments [see Hobbs (1974) and Petrenko and Whitworth (1999)], e.g., from amorphous ices, high-pressure ices, from water vapor or from the liquid in confined systems, or by hyperquenching (Kohl, Mayer, and Hallbrucker, 2000). More recently it was established that it can also form from decomposing gas hydrates (Kuhs et al., 2004). It may thus play a role in transitions within the complex phase diagram of water. Although ice Ih is the thermodynamically stable phase, the formation of ice Ic is understandable due to its lower nucleation barrier (Kobayashi and Kuroda, 1987) and can be seen as a manifestation of Ostwald's step rule.

Its idealized structure represents the crystalline water arrangement with the highest conceivable symmetry. The idealized structure is topologically related to the silicate structure of cristobalite (while common hexagonal ice is related to the structure of tridymite). Yet this idealized structure has never 
been observed in experiments. After the first identification of ice Ic (König, 1943) it was realized by Shallcross and Carpenter (1957) that the diffraction pattern of ice Ic had hexagonal components, wrongly interpreted by them as an admixture of ice Ih. Many attempts were made to explain the origin of these hexagonal features and it was realized early on that there is a gradual transition from ill-defined ice Ic into well-crystallized ice Ih. Yet the transition temperature (range) differed, partly owing to differences in the sensitivity of the method used for detection. It turns out that a suggestion by Kuhs, Bliss, and Finney (1987) set the correct entry point for a final quantitative description of the real structure of ice Ic by ascribing the odd features in the diffraction pattern to stacking faults combined with particle size broadening, the latter owing to the small crystallite size, typically of the order of a few hundred $\AA$. This idea was taken up by Morishige and Uematsu (2005) and finally quantitatively treated by Hansen, Koza, and Kuhs (2008) and Hansen et al. (2008). The model of the latter authors allows for a detailed description of differences in the stacking-fault pattern that appear to occur as a function of the formation procedure, of temperature and, for isothermal systems, also as a function of time. Thus, once formed, ice Ic can be considered as a material under ongoing reconstruction in terms both of stacking-fault pattern and of crystallite size. Its final destination is certainly ice Ih, yet to reach this state the material appears to need warming up to temperatures in the vicinity of $240 \mathrm{~K}$ (Kuhs et al., 2004); at lower temperatures transformations may take longer than the usual laboratory time scales and eventually may even get stuck with remaining faults. The energy difference between ice Ic and ice Ih is very small, some $50 \mathrm{~J} \mathrm{~mol}^{-1}$ (Handa, Klug, and Whalley, 1986), as is the stacking-fault energy, estimated to be on the order of $0.1-0.3 \mathrm{~mJ} \mathrm{~m}^{-2}$ (Oguro and Hondoh, 1988); yet the annealing of stacking faults is linked to the migration of point defects [see, e.g., Petrenko and Whitworth (1999)] with their much higher activation energies. Thus there is not only one ice Ic, but a range of cubic ices with varying degrees of (im)perfection and varying particles sizes, all annealing as a function of both time and temperature, yet seemingly doing so in different ways depending on the initial formation conditions.

Modeling tools now allow for the study of processes involving ice Ic in any necessary detail (Hansen, Koza, and Kuhs, 2008; Hansen et al., 2008). However, not all the mysteries of ice Ic have been solved. It is at present unclear whether and to what extent the lattice spacing is affected by the stacking-fault sequences or by the local strain; the latter may well arise from incoherent interfaces between the ice Ic nanocrystallites (Johari, 1998). The local molecular geometries arising from the crystallographic disorder of the oxygen atoms (see above) are considerably less well known than in ice Ih. On a nanoscopic scale it appears quite possible that between the stacking-faulty crystalline regions some interfacial, topologically disordered material, possibly with some enhanced mobility, persists in (sub)nanometric patches, as has been suggested by recent molecular-dynamics (MD) computer simulations (Moore et al., 2010; Moore and Molinero, 2011). It is not straightforward to confirm this experimentally, as small amounts of such disordered material are not easily detected in the presence of a dominant crystal- line component. It should also be noted that these computer simulations reach "only" time scales of several hundred nanoseconds and the material obtained would very likely undergo further rather rapid annealing and crystallization on time scales beyond that limit.

As the ice microstructure, in particular, its surface features, has implications for astrophysical and atmospheric considerations (see Secs. III and IV), it is important to shed further light on this issue. Major contributions can be expected from in situ $\mathrm{x}$-ray and neutron scattering (diffraction and small angle scattering) combined with ex situ scanning electron microscopy (SEM) and in situ environmental scanning electron microscopy (ESEM) work. We are only just beginning to see how this complex material behaves and are still far from any detailed understanding. Clearly, the multiscalar microstructure and its complex transformation kinetics cannot be disentangled in a single experiment; its complex nature rather needs a long-term commitment.

\section{Amorphous ices}

\section{Crystalline and amorphous structures}

In Sec. II.A we rationalized the structures of the crystalline ices phases in terms of the basic tetrahedral unit of Fig. 2, in which a central water molecule donates two hydrogen bonds to two of its four neighbors and accepts two hydrogen bonds from the other two. The ways in which these (varyingly distorted) units connect together give rise to the different ice structures that pepper the phase diagram shown in Fig. 1.

A necessary characteristic of all these structures, as with all crystalline structures, is that the molecules be arranged in a regular, repeating way. Formally, we can define a unit cell for each crystalline structure (see, for example, that of ice XIII in Fig. 5) and then, by repeating this unit cell indefinitely in three dimensions, generate the structure of the whole crystal. What happens when we remove this constraint of crystallinity? What kinds of structures can we obtain, and can such structures exist in reality? The answer to the second question is yes, and the resulting amorphous ices have been known for many years. Their structures have, however, only been definitively determined in the last decade, and as in the crystalline case, there remain major unanswered questions concerning some aspects of amorphous ices.

Before looking at the structures of amorphous ices, it is worth discussing amorphous solids in general. As we reduce the temperature of a liquid (which again has a noncrystalline arrangement of molecules), we usually come to a temperature (neglecting questions of supercooling) at which the liquid crystallizes and a crystalline phase is formed, just as ice Ih forms in our environment (again ignoring supercooling) at $0{ }^{\circ} \mathrm{C}$. In many liquids, however, if we cool quickly enough, the molecules may not have sufficient time to rearrange themselves into the equilibrium crystalline structure and a noncrystalline glass may form, an obvious example being the cooling of silica-based liquids to form the glass with which we are all familiar. Silica-like water-has a local tetrahedral four-coordinated molecular-level structure, and the atoms in the glass maintain the fourfold local coordination as found in the various crystal forms of silica, but without a regular 
(a)

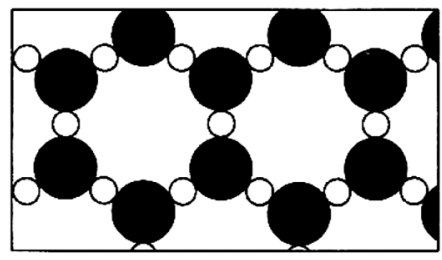

(b)

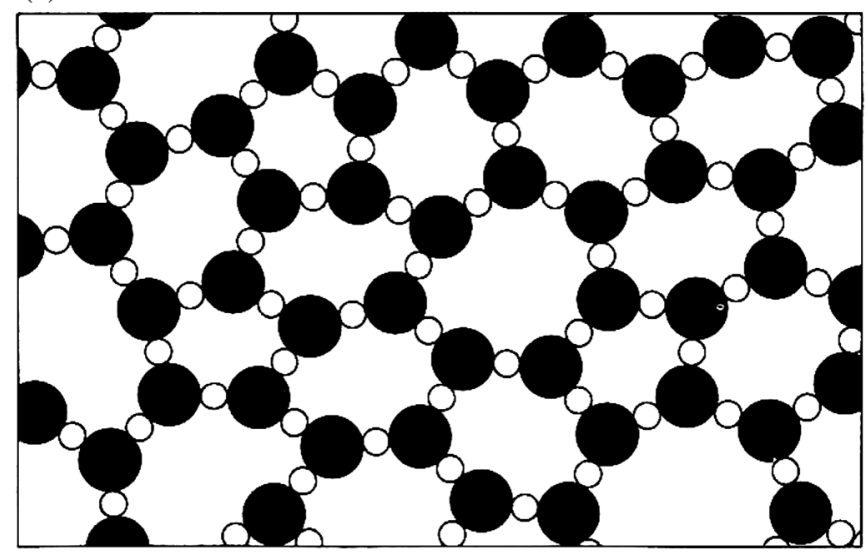

FIG. 6. Two-dimensional analogs of (a) crystalline and (b) amorphous "glassy" solids made up of threefold coordinated basic molecular units.

repeating arrangement of the constituent atoms (see Fig. 6 for a two-dimensional analog).

The same can happen with water: We can form a noncrystalline arrangement of water molecules, in which the basic fourfold local motif is expected to be retained, although the rates of cooling have to be very rapid indeed. And there are other ways apart from cooling the liquid in which a noncrystalline ice can be made. Currently, it is generally recognized that there are five different kinds of amorphous ice, distinguished originally by their methods of preparation.

\section{Formation and structures of amorphous ices}

The first kind, amorphous solid water (ASW), was produced initially by Burton and Oliver (1935) by depositing water vapor on a cold substrate. As formed, this material is microporous (Mayer and Pletzer, 1986), but can be consolidated to a reproducible density by annealing or sintering at a temperature below about $110 \mathrm{~K}$. Another noncrystalline form, hyperquenched glassy water (HGW), can be prepared by very rapid cooling of liquid droplets by either injecting a fine water spray into a cryoliquid or supersonically impacting micronsized droplets onto a substrate at $77 \mathrm{~K}$ (Mayer and Brüggeller, 1982; Mayer, 1985). Both the above forms have the same atomic density of 0.0937 atom $\AA^{-3}$ (approximately $0.94 \mathrm{~g} \mathrm{~cm}^{-3}$; there is good reason to use atomic densities as the density depends on the isotopic composition of the sample and the experimental neutron-scattering work that is used to determine the structures uses different isotopic compositions). Pressurizing crystalline ice Ih to about $1 \mathrm{GPa}$ at $77 \mathrm{~K}$ results in another apparently amorphous form (Mishima, Calvert, and Whalley, 1984). This was labeled high-density amorphous ice (HDA) on account of its significantly higher density of 0.117 atom $\AA^{-3}\left(\sim 1.15 \mathrm{~g} \mathrm{~cm}^{-3}\right)$. Controlled heating of HDA recovered to ambient pressure produced a "low-density amorph" (LDA) above about $120 \mathrm{~K}$, which has the same density as ASW and HGW (Mishima, Calvert, and Whalley, 1985). A very high-density form (VHDA, atomic density 0.125 atom $\AA^{-3}, \sim 1.26 \mathrm{~g} \mathrm{~cm}^{-3}$ ) was discovered more recently by isobaric heating of HDA under pressure (Loerting et al., 2001).

Although there has been an expectation for many years that the atomic-level structures of the three forms of amorphous ice produced differently but having the same density (ASW, $\mathrm{HGW}$, and LDA) are similar, it is only in the last few years that neutron-diffraction techniques established this structural identity (Bowron et al., 2006). The water molecules in these three amorphous forms are almost perfectly fourfold coordinated, with each water molecule surrounded on average by four hydrogen-bonded neighboring water molecules at distances of between 2.5 and $3.3 \AA$. The neutron-scattering results additionally tell us that the mean $\mathrm{O}-\mathrm{O}-\mathrm{O}$ angle in the LDA network is $\sim 111^{\circ}$, close to the ideal tetrahedral angle of $109.47^{\circ}$. We can thus conclude that the short-range structures of LDA, ASW, and HGW all relate closely to a hydrogen-bonded tetrahedral network of water molecules.

The structures of the denser forms, HDA and VHDA, are somewhat more complex. A coordination-number analysis for these ices again shows that each oxygen atom is immediately coordinated by approximately four neighboring water oxygen atoms, situated in the distance range from 2.5 to $3.1 \AA$. In contrast to the lower-density forms such as LDA, however, where the number of first neighbors remains at 4 up to a distance of $3.4 \AA$, by this point the water-molecule oxygen coordination number in HDA rises to $\sim 5$ and to a value of $\sim 6$ in VHDA. Again the neutron data tell us that each water oxygen is hydrogen bonded to two hydrogen atoms, confirming that we have a four-coordinated hydrogen-bonded network. The higher densities of these two forms thus appear to be determined by molecules that are farther away from a typical "central" molecule moving closer in toward that central molecule, although not being hydrogen bonded to that central molecule. Although these "closer in" molecules were labeled "interstitial" molecules when they were first identified (Finney, Bowron et al., 2002; Finney, Hallbrucker et al., 2002), it is important to emphasize that they themselves still appear to be hydrogen bonded to four neighbors in (distorted) tetrahedral geometries, and are not interstitial in the common understanding of the term of being embedded separately in the main matrix structure (which would be a crystalline lattice in the crystalline case).

So, just as with the crystalline ices, all these amorphous ice forms have structures that are essentially made up of the four-coordinated basic motif of Fig. 2, although in the amorphous case these units are connected together in a noncrystalline, nonrepeating way. Moreover, as in some of the higher-pressure crystalline phases, the degree of distortion of this basic structural unit appears to be increased in the higher-density forms HDA and VHDA. In these ices, this distortion appears to be high enough to allow molecules that would in the case of, for example, LDA be non-hydrogenbonded second neighbors to be "pushed in" to distances from the central molecule that are of the same order as those of the hydrogen-bonded first neighbors, yet which remain nonhydrogen bonded to that central molecule. 
Thus, from a situation a decade ago in which we were unsure of the structures of the four amorphous forms ASW, HGW, LDA, and HDA, we moved to one in which the essential structural identity of the first three, equal-density, forms has been established as being essentially a random network of four-coordinated water molecules. Moreover, we know that the higher-density form, HDA, although still having a four-coordinated structure, has on average an additional molecule almost as close in as each of the hydrogen-bonded first neighbors. The more recently discovered very-highdensity form, VHDA, appears to have a structure similar to that of HDA, though with additional occupation of the socalled interstitial location.

\section{Some outstanding questions}

Although the amorphous ice situation has been simplified in some ways, in other respects it has become more complex, as a cursory look at the literature since 2002 will quickly demonstrate. A number of interesting questions remain to be addressed.

The first of these concerns detailed structural characterization: What is the detailed relationship between the networks of the low-density and high-density forms? How are the water molecules in the interstitial sites connected through the hydrogen-bonded network to the molecule at the tetrahedral central site? Is this connectivity the same as in, e.g., LDA with the interstitial being simply a previously more distant second neighbor that is pushed in closer without any change in network connectivity, or has a change in the network topology occurred? If the first option is the case, then LDA, HDA, and VHDA can be seen as essentially the same topological structure, with just increasing distortion of the basic tetrahedral unit allowing us to move from the low-density structure to the higher-density ones. If the second is true, then how is the network connectivity changed? Are there instances in which hydrogen-bond linkages pass through ring structures formed by other water molecules, as observed in the interpenetrating networks found in the higher-pressure ices IV, VI, VII, and VIII? And if these higher-density amorphous forms do have a distinctly different network connectivity from the low-density forms, can this be considered a distinctly different water structure that could relate to the continuing debate (Mishima and Stanley, 1998) about liquid water itself being able to exist in two distinctly different structural forms?

Whereas the above structural questions can be, and, we hope, are being, answered by a detailed examination of the network structures of the high- and low-density forms (Loerting, Bowron, and Finney, 2010), there are further complexities that are more challenging to resolve. For example, structural annealing studies of the conversion of the high-density forms into the low-density forms by heating have been interpreted as suggesting the existence of a family of high-density structures (Tulk et al., 2002; Koza et al., 2003) and also the possibility of a range of low-density forms [Koza et al., 2003; see also Koza (2009)]. However, this picture has been challenged by subsequent studies (Mishima and Suzuki, 2002; Nelmes et al., 2006; Winkel et al., 2008) that demonstrated that if care is taken to anneal the high-density amorphous ice forms to a structure that is thermodynamically metastable with respect to the applied pressure and temperature conditions of ice formation, the complexity of the high-density to low-density transition is simplified. Subsequently, the annealed form of HDA has been relabeled expanded HDA (eHDA) (Nelmes et al., 2006).

Furthermore, a recent computer-simulation study raised the suggestion of a second distinct low-density form of amorphous ice (Guillot and Guissani, 2003). This challenges the prevailing experimental view of one low-density amorphous structure (Bowron et al., 2006) which is thought to be the metastable minimum structure. Within the framework of the simulation, a form labeled $\mathrm{LDA}_{\mathrm{I}}$ was produced by warming the model of HDA, while a second slightly more dense ice $\mathrm{LDA}_{\mathrm{II}}$ was produced by warming the model of VHDA. Although interesting, these suggestions seemed to be at odds with existing experimental observations that the density of LDA produced by warming either eHDA or VHDA samples was found to be the same, at 0.0937 atom $\AA^{-3}$ (Debenedetti, 2003; Loerting and Giovambattista, 2006). However, initial neutron-scattering experiments (Winkel et al., 2009) indicated that there exist at least two structural forms of low-density amorphous ice, where the primary difference in the structures occurs on an extended length scale in the hydrogen-bonded network of molecules. LDA and $\mathrm{LDA}_{\mathrm{II}}$ are not seen to be distinct forms of ice in the way that LDA, HDA, and VHDA differ, but rather are two closely related, but kinetically trapped, forms of what could be considered the true metastable low-density amorphous ice characterized by an atomic density of 0.0937 atom $\AA^{-3}$.

A further question that remains to be answered concerns the relationship between the structure(s) of amorphous ices and the structure of liquid water. The fact that LDA can be formed by rapid cooling of the liquid suggests a structural continuity with the liquid, and this is generally accepted to be the case. However, there has been much speculation concerning the possible existence of more than one liquid phase of composition $\mathrm{H}_{2} \mathrm{O}$, possible liquid-liquid immiscibility, and the existence of a second critical point [see, for example, Poole et al. (1992), Debenedetti (1996), and Mishima and Stanley (1998)]. Noting the polyamorphism exhibited by the existence of more than one amorphous ice, with the data currently available suggesting the existence of three structurally distinguishable amorphous ices (LDA, HDA, and VHDA) (Loerting et al., 2011), HDA (Mishima and Stanley, 1998), and, since its discovery in 2001 (Loerting et al., 2001), VHDA (Finney, Bowron et al., 2002) have been proposed as possible glassy analogs of the putative "high-density liquid." Such hypotheses do, however, remain hypotheses at the present time.

We concluded the section on crystalline ices with the phrase "strange indeed." The amorphous ices situation qualifies equally for this description-and perhaps more so. Clearing up the current confusion in amorphous ices-where we are of necessity dealing with nonequilibrium systems, complicated further by structural characterization problems made more difficult by the lack of an ordered crystalline structure-is perhaps an even more challenging problem than that of finally sorting out the ice phase diagram. 


\section{Polycrystallinity and interfaces}

A discussion of the structure and phase morphologies of ice is incomplete without addressing the important role that polycrystallinity, surfaces, and interfaces play. Many of the important physical and chemical processes that are discussed in depth throughout this work are heavily influenced by ice grain structure and the character of ice surfaces and phase transitions. For example, in Sec. V.A.3, we discuss how the grain size of ice in glaciers changes with time, and in Sec. V.B the role of grain boundaries on the uptake of atmospheric trace gases is noted.

Fundamentally, ice is very similar to a wide range of other polycrystalline materials, but is of particular interest because of its geophysical importance. Furthermore, many interesting and important effects result from the fact that the ice around us exists at temperatures and pressures very close to where we expect phase coexistence. Detailed and authoritative reviews have addressed many problems of grain structures, surfaces, and phase transitions in ice (Dash, Rempel, and Wettlaufer, 2006), metals (Smith, 1964), and other materials (Luo and Chiang, 2008). Here we simply summarize the most important aspects of these various behaviors.

\section{Ice-e pluribus unum}

Naturally occurring ice, like most crystalline materials, is usually made up of many crystallites, or grains. By the time it reaches the ground, even meteoric ice that begins as single crystals in the atmosphere is often polycrystalline. As a result, when we discuss the morphology of ice, in addition to the molecular structure of single crystals, the granular structure of the bulk can be very important.

Ice grains can have a range of sizes that strongly depends upon the thermodynamic history of the particular sample. For example, within polar ice sheets crystal diameters range from less than $1 \mathrm{~mm}$ to more than $1 \mathrm{~m}$ (Thorsteinsson, Kipfstuhl, and Miller, 1997 and Sec. V.A.3). When visualizing hexagonal ice crystals we can take advantage of their optical anisotropy; under cross-polarized light individual crystal domains are clearly seen (see Fig. 7). With this technique generally even very small grains can be distinguished with a micro-

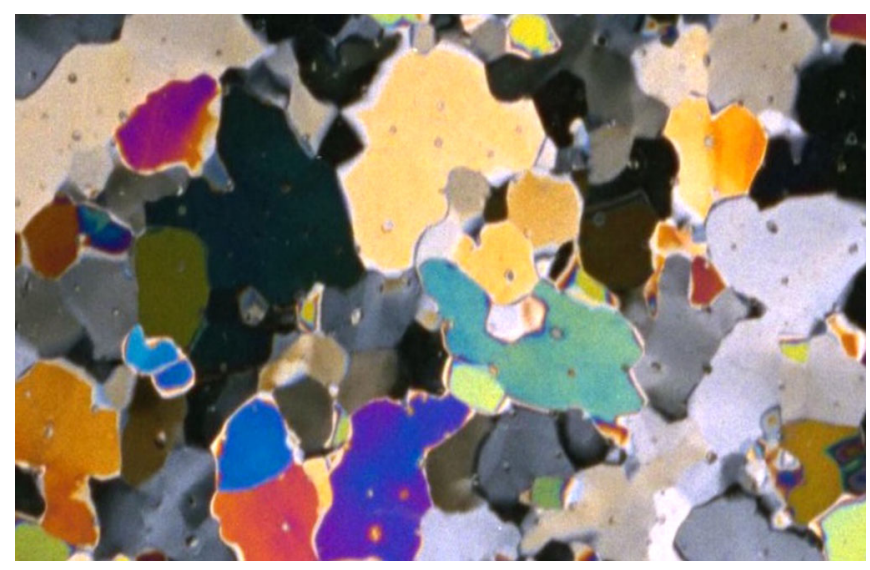

FIG. 7 (color online). A thin $2 \mathrm{~cm}$ by $3.7 \mathrm{~cm}$ polycrystalline ice section seen under cross-polarized light. Individual crystals are seen in different shades and colors due to the birefringent nature of ice. scope. In ice and other materials grain structure is observed either on exposed surfaces or, as we see in Fig. 7, by slicing thin sections and examining the resulting two-dimensional structure. At such length scales, in its two-dimensional form the underlying molecular structure of ice is not evident, and the individual crystals do not form standard polyhedra. Instead, the discontinuities between crystals of different orientations stand out.

When ice is warmed and the bulk melting temperature is approached, the atomic and molecular disorder present at low temperatures grows to produce macroscopic liquid layers at crystal interfaces. The planar structure between two single grains is referred to as a grain boundary. Where three grains intersect veins form, and where four or more crystals come together nodes are formed. These structures are themselves surfaces, internal to bulk polycrystals, and are discussed in more detail in Sec. II.D.2.

In macroscopic terms, the hexagonal symmetry of molecular ice is described by prism facets surrounding an optical axis of symmetry, the $c$ axis, which is perpendicular to the basal plane. The basal plane is characterized by a higher roughening temperature than the prism faces and therefore is often observed as a smooth surface (Wilen and Dash, 1995; Furukawa and Nada, 1997a). In the atmosphere, crystal shape is generally determined by supersaturation (see Sec. IV.D.1), but in bulk polycrystals many postnucleation processes determine the evolution of crystal shape and size. In systems under stress $c$ axes are known to rotate in order to align themselves in the direction of deformation (Paterson, 1994). In polycrystalline systems with a distribution of grain sizes, grain-boundary migration leads larger crystals to absorb smaller crystals, and over time the average grain size increases (Alley, 1992). On the other hand, when dislocations are readily produced, they may accumulate to form new grain boundaries, a process called polygonalization. In systems where large strains exist, completely new grains may nucleate in a process termed recrystallization (Alley, 1992; Paterson, 1994). Bubbles, impurities, and other discontinuities within the crystal matrix affect each of these processes in complicated and nontrivial ways. The geometry and spongelike nature of fallen snow add complexity due to the presence of air and water vapor. Just as surface and strain gradients drive grain growth in solid ice, snow responds to vapor gradients. Fundamentally the same morphological processes affecting ice-crystal character are important to snow as it compacts and densifies, forming firn and eventually glacial ice (see Sec. V.A.3).

Often in the laboratory idealized single crystals with measured or controlled size, shape, and crystal orientation are examined. However, when working with polycrystalline ice samples, such as those described in Secs. III and IV, the effect of the polycrystalline morphology of ice must always be acknowledged.

\section{Surfaces and interfaces}

In addition to the free surface, the grain boundary, vein, and node interfaces internal to bulk polycrystalline ice play important physical and chemical roles. Surfaces are where phase change is initiated and are regions of enhanced impurity concentration. Below the bulk melting temperature 
of ice, these interfaces can be disordered, even liquid, at equilibrium.

With increasing temperature the outermost molecular layers of an ice surface in contact with a substrate or vapor can become increasingly mobile, and under the right circumstances form a premelted liquid layer [for a complete review, see Dash, Rempel, and Wettlaufer (2006)], which is of importance to the human activities of skiing, snowballing, skating, etc. The morphology of the surface as it nears the melting temperature depends upon the specifics of the system and is rooted in the strength of the intermolecular interactions within and between the various materials. The same intermolecular interactions link the physics of melting with wetting behavior (Schick, 1990; French et al., 2010). The existence of distinct droplets versus spreading liquid layers on surfaces is related to the relative cohesion versus adhesion of the material. Droplets form when cohesion dominates, but when adhesion wins the liquid thins and spreads over the solid. The balance between these effects represents the range of possible melting behaviors from partial to complete melting. Likewise the microscopic details of melting change with increasing temperature as surface structure breaks down and disorder and mobility increase. In this transition liquidlike structures that retain some of the underlying crystal order, called quasiliquid layers, represent an intermediate state between the solid and bulk liquid. In practice, the distinction between liquid and quasiliquid can be difficult to make and often experiment and theory require us to make assumptions concerning the physical properties (e.g., density, viscosity, index of refraction) of such layers. In these cases, the assumption of bulk liquid properties represents one limiting case, which for many properties has been shown to be representative even for very thin layers [see, e.g., Raviv, Laurat, and Klein (2001)].

Thus a liquid or quasiliquid surface layer will exist, in the bulk-solid region of the phase diagram, in thermodynamic equilibrium, if by being there the total free energy of the system is reduced. Elbaum, Lipson, and Dash (1993) demonstrated that the surface melting of ice in equilibrium with its vapor can vary between partial and complete, but for systems with even small impurity levels complete melting is observed. Ice surface and interfacial melting can be well modeled theoretically using unretarded van der Waals forces, whose long-range potential falls off quadratically with distance (Dash, Rempel, and Wettlaufer, 2006). This yields an expression for the thickness $d$ of the liquid layer as a function of temperature $T$ relative to the bulk melting temperature $T_{m}$,

$$
d=\left(\frac{-2 \Delta \gamma \sigma^{2}}{\rho_{1} q_{m}}\right)^{1 / 3}\left(\frac{T_{m}-T}{T_{m}}\right)^{-1 / 3},
$$

where $\rho_{l}$ is the density of the bulk liquid, $q_{m}$ is the latent heat of transformation, and $\sigma$ is a constant on the order of a molecular diameter. The difference between the wet and the dry interfacial free energy is $\Delta \gamma=\gamma_{s l}+\gamma_{l v}-\gamma_{s v}$, where each $\gamma$ is an interfacial free energy per unit area with the subscripted letters defining the type of interface ( $s$, solid; $l$, liquid; $v$, vapor). In the case of interfacial melting the vapor phase is replaced by a substrate. Modeled and observed thicknesses range from 1 to $20 \mathrm{~nm}$ and tend to (a)

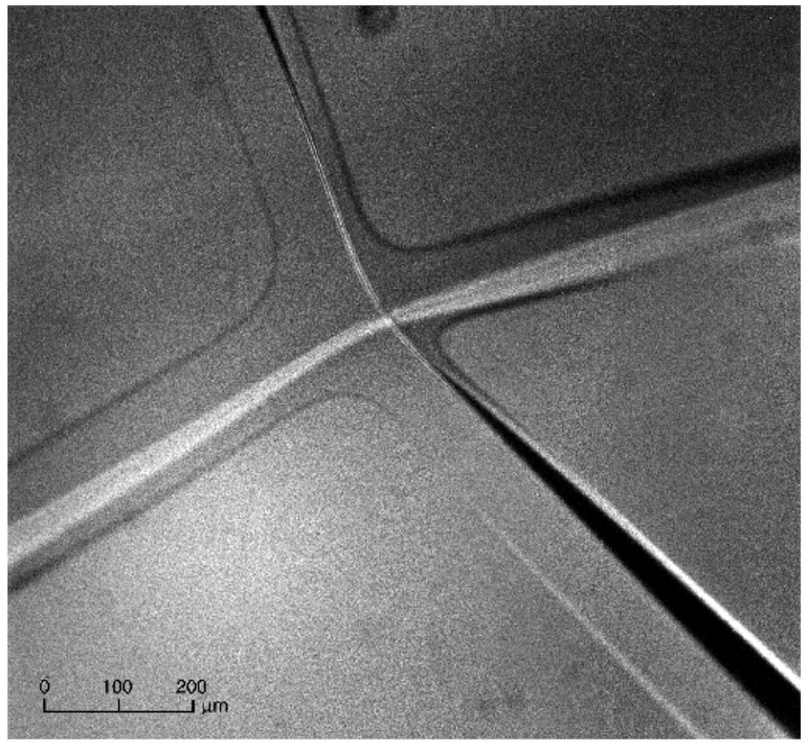

(b)

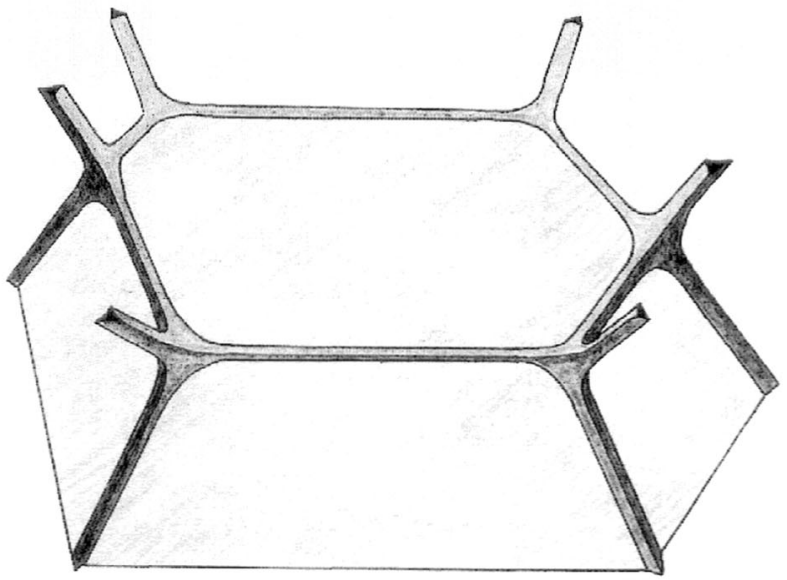

FIG. 8. The vein-node network in polycrystalline ice: (a) Micrograph published originally by Mader (1992); (b) schematic from Nye (1992), modified from Smith (1948). Together they appear as Fig. 11 in Dash, Rempel, and Wettlaufer (2006).

diverge as the melting temperature is approached (Elbaum and Schick, 1991; Elbaum, Lipson, and Dash, 1993; Sadtchenko and Ewing, 2002).

Similar to the free surface, within ice, veins and nodes of liquid water form in equilibrium below the bulk melting temperature; see Fig. 8. However, in this case they exist largely because these interfaces have a high degree of curvature. Thermodynamically, the energy cost of a curved solid interface is related to the surface energy per unit area $\gamma_{s l}$ and interfacial curvature $\mathcal{K}=1 / r$, vis-à-vis the GibbsThomson effect (Thomson, 1871), which is also important in nucleation (see Sec. IV) and the bubble and crystal coarsening of Ostwald ripening (Hillert, 1965; Avron and Levine, 1992). In the vein and node network the Gibbs-Thomson effect results in a depression of the equilibrium melting temperature that can be written as

$$
T_{m}-T=\frac{\gamma_{s l} T_{m}}{\rho_{s} q_{m}} \frac{2}{r},
$$


where for simplicity the solid is modeled as a sphere of radius $r$ (positive when the interface is convex into the melt), and $\rho_{s}$ is the bulk density of the solid. As $r$ increases the magnitude of $T_{m}-T$ decreases, and approaches zero for flat interfaces. Again the existence of liquid within the bulk-solid polycrystalline ice is the thermodynamically favorable state. In ice the liquid veins and nodes threading throughout the volume can be tens to hundreds of micrometers wide (see Fig. 8).

Grain boundaries are particularly important to understanding polycrystalline morphology. In most materials they are challenging to access, yet they represent the dominant surface area of a polycrystal. At these boundaries curvature disappears and the dispersion forces are attractive for systems of like materials straddling an interface. However, the crystalline discontinuity suggests that some level of molecular or atomic disorder must exist between differently oriented grains. For a range of materials and interaction potentials, grain-boundary disorder has been theoretically and numerically modeled [see, e.g., Broughton and Gilmer (1986), Mellenthin, Karma, and Plapp (2008), and Schick and Shih (1987)]. Thomson, Benatov, and Wettlaufer (2010) and Benatov and Wettlaufer (2004) suggest that wetted grain boundaries in impurity-doped ice may be measurable using optical light-scattering techniques (Thomson, Wettlaufer, and Wilen, 2009). Their experimental observations seem to support the idea that in impurity-rich systems grain-boundary disorder can be stabilized with thicknesses of tens to hundreds of monolayers. In general, experiments on, and models of, grain boundaries show agreement that grain-boundary morphology is very sensitive to the specific interactions within a system. In particular, in ice, surface charge and impurity loading strongly affect grain-boundary equilibrium.

All ice surface effects are strongly influenced by the presence of impurities. Ice is a particularly efficient segregator of impurities, and as a result impurities in water are strongly concentrated at ice surfaces, rather than being incorporated by the solid lattice, during freezing. In addition to the classical colligative effect of depressing the equilibrium melting temperature, enhanced impurities can drive instabilities in freezing fronts (Wettlaufer, 1994), alter surface charge densities (Jackson et al., 1997), and affect the intermolecular interactions of a system (Dash, Rempel, and Wettlaufer, 2006). Impurities interacting with ice are particularly important in geophysical environments, where they are ubiquitous, and much of the discussion in this work centers on their physical and chemical importance in various settings. The fact that biological processes also take place at snow and ice surfaces was recently emphasized (Ariya et al., 2011).

Ice is an interesting material on many levels. Its molecular structure and phase morphologies inspire a multitude of science. In our natural environment ice characteristically exists as a polycrystalline material near its triple point, resulting in diverse behavior with macroscopic importance. As stress, temperature, composition, and pressure of the environment vary so too do the structural and surface responses of ice. From the molecular to the macroscopic, the structure of ice is an important variable underlying behavior in the cryosphere.

\section{ASTROPHYSICAL ICE}

The ice did not have to be quarried. It existed in proper chunks in the rings of Saturn. That's all the rings were-pieces of nearly pure ice, circling Saturn. So spectroscopy stated and so it had turned out to be. He was standing on one such piece now, over two miles long, nearly one mile thick. It was almost half a billion tons of water, all in one piece, and he was standing on it.

\section{Isaac Asimov, The Martian Way}

In astrophysics "ices" are defined as those molecular species that are liquid or gaseous at room pressure and temperature $\left(1 \mathrm{~atm}\right.$ and $\left.25^{\circ} \mathrm{C}\right)$ but are solids under the physical conditions of the astrophysical environment in question. Water ice is the most abundant such ice, and here, naturally, we concentrate on it, but in astrophysics one may often be interested in its abundance relative to other species and in mixtures of water ice with other ices.

Water ice is present on many objects in the Solar System (Cartwright, 2007; Bell, III, 2009, and references therein). In addition, the occurrence of frozen volatile molecules in these objects is a matter of current discussion. In Table I we present a summary of the ice species detected and hypothesized to exist on different bodies in the Solar System. At present the Messenger probe orbiting Mercury is providing indications that ice may be present in polar craters there (Schilling, 2011). Although Venus has a cryosphere (Bertaux et al., 2007) in its upper atmosphere, if there is ice on Venus, it can only be in small quantities in the atmosphere above $80 \mathrm{~km}$ at night; present results are equivocal (Fedorova et al., 2008; Montmessin, 2102). Perhaps one of the most controversial issues is the possible presence of water on the Moon. Many articles have provided pros and cons for this case based on different interpretations of the available evidence through time. The latest results based on the analysis of debris following an impact on the lunar surface provide strong evidence for the presence of water (Colaprete et al., 2010), but ice has not yet been detected. The possibility that Earth may possess a faint ring, responsible for slight climate alterations, has even been raised in the past (O'Keefe, 1980) and in recent investigations (Hancock and Povenmire, 2010), although the existence of the ring, and of its being composed of ice, seems rather dubious. The polar regions of Mars are rich in ices (mainly carbon dioxide and water), but frozen aerosols containing these species are also detected in the Martian stratosphere (McCleese et al., 2010). Recently two teams (Campins et al., 2010; Rivkin and Emery, 2010) provided evidence of water ice on the asteroid 24 Themis, thus rekindling the discussion of the possible water delivery to Earth from these objects, abundant in the main asteroid belt, between Mars and Jupiter. Even more recently, Licandro et al. (2011) contributed to this argument with findings of water and organics on the asteroid 65 Cybele. The satellites of the outer planets, Jupiter and beyond, are dominated by water ice. Europa and Enceladus show fractured surfaces formed by blocks of water ice, which bear some similarity to icy surfaces on Earth. As Asimov anticipated, the rings of Saturn 
TABLE I. Objects in the Solar System on which ice has been detected or for which (denoted with *) its presence has been hypothesized.

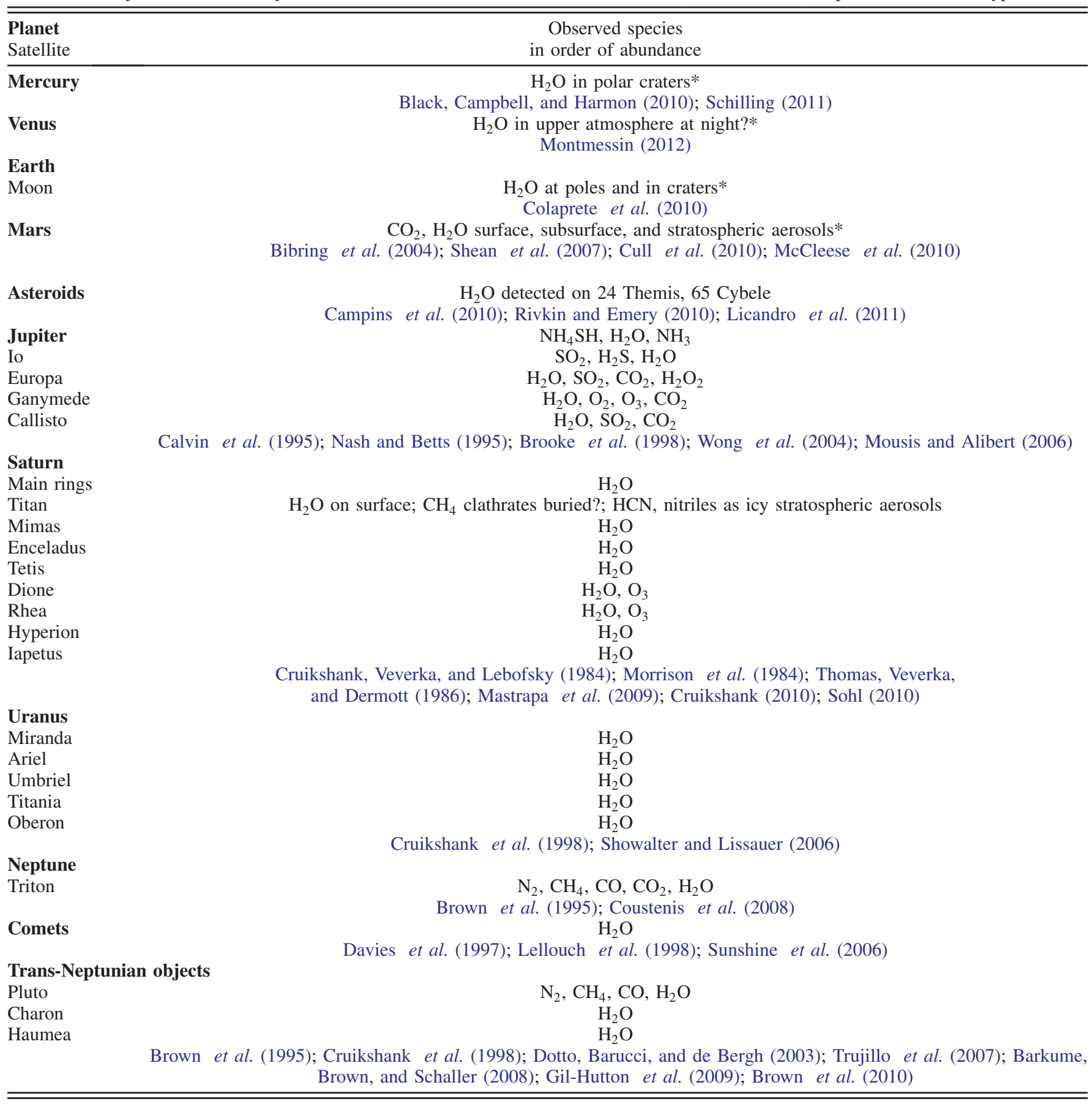

and other bodies of the outer Solar System, including the ring systems of Jupiter, Uranus, and Neptune, are composed of $95 \%$ water ice (Heißelmann et al., 2010) [however, the sizes of the fragments seem to be rather less than Asimov surmised (Cuzzi et al., 2009)]. On Pluto and the other so-called transNeptunian objects, a class of numerous small bodies not yet well investigated, some ice species have been detected. The composition of these far-off objects is not fully understood, but infrared reflectance data point to the existence of water and possibly methane ices. Water ice is found in both crystalline and amorphous phases, and models are employed to simulate the observations based on laboratory optical indices of these systems (Mastrapa et al., 2009). Comets contain a large inventory of ices that are observed after their sublimation during comet passages near the Sun, but their presence is often masked by devolatilized surface materials (organics and/or silicates). Experiments related to the sublimation of ice mixtures are then particularly relevant in this context; see Sec. III.A. The existence of ice on the newly detected exoplanets is already being considered; observations are being carried out to investigate this possibility, for instance, through surface albedo and color (Cahoy, Marley, and Fortney, 2010).

Ices in the Solar System suffer from energetic processing by solar photons, solar wind and solar flares ions, and galactic cosmic rays. Furthermore, satellites moving in the magneto- 
TABLE II. Molecular species detected on icy mantles in dense interstellar clouds and their relative abundance.

\begin{tabular}{|c|c|c|}
\hline Species & Relative abundance & Reference \\
\hline $\mathrm{H}_{2} \mathrm{O}$ & 100 & \\
\hline $\mathrm{CO}$ & $0-40$ & Chiar et al. (1994); Boogert and Ehrenfreund (2004) \\
\hline $\mathrm{CH}_{3} \mathrm{OH}$ & $2-31$ & Allamandola et al. (1992); Dartois, Schutte et al. (1999); Boogert et al. (2008) \\
\hline $\mathrm{NH}_{4}^{+}$ & $1-26$ & Boogert and Ehrenfreund (2004); Boogert et al. (2008) \\
\hline $\mathrm{CO}_{2}$ & $10-25$ & Gerakines et al. (1999); Zasowski et al. (2009) \\
\hline $\mathrm{CH}_{4}$ & $0.3-23$ & Lacy et al. (1991); Boogert et al. (1997); Zasowski et al. (2009) \\
\hline $\mathrm{HCOOH}$ & $1-16$ & Boogert et al. (2008) \\
\hline $\mathrm{NH}_{3}$ & $1-10$ & Lacy et al. (1998); Williams et al. (2007) \\
\hline $\mathrm{OCN}^{-}$ & $1-8$ & Tegler et al. (1995) \\
\hline $\mathrm{H}_{2} \mathrm{CO}$ & $0-7$ & Schutte et al. (1996); Zasowski et al. (2009) \\
\hline $\mathrm{SO}_{2}$ & $0.3-1.7$ & Boogert et al. (1997); Zasowski et al. (2009) \\
\hline OCS & $0.04-0.4$ & Palumbo, Geballe, and Tielens (1997); Williams et al. (2007) \\
\hline
\end{tabular}

sphere of their planet are exposed to the effects of magnetospheric ions [see, e.g., Johnson (1990) and Strazzulla and Johnson (1991)]. Just one example is the case of hydrogen peroxide $\left(\mathrm{H}_{2} \mathrm{O}_{2}\right)$ that has been detected on Europa (Carlson et al., 1999). Comparison with laboratory spectra indicates a surface concentration of about $0.13 \%$, by number of molecules, relative to water ice. Europa is immersed in a highly energetic plasma environment originating in Jupiter's magnetosphere (Cooper et al., 2001). Based on the results of several experiments, radiolysis is supposed to be the dominant formation mechanism of hydrogen peroxide on Europa (Moore and Hudson, 2000; Gomis et al., 2004). Laboratory experiments also suggest a patchy distribution of $\mathrm{H}_{2} \mathrm{O}_{2}$ on Europa. This result could support the hypothesis of a radiation-driven ecosystem on Europa based on the availability of organic molecules and oxidants such as hydrogen peroxide (Chyba, 2000). More results are available through the numerous missions currently deployed to several planets, especially Mars; the interested reader is advised to visit the Web pages of NASA, ESA, and other space agencies.

Beyond the Solar System, the interstellar medium contains about $10 \%$ of our galaxy's mass. It is mainly composed of atomic and molecular gas species $(99 \%$ by mass, of which $70 \%$ is $\mathrm{H}, 28 \% \mathrm{He}$, and $2 \%$ are heavier elements), and dust ( $1 \%$ by mass) and is pervaded by photon and particle radiation. The gas is characterized by two main parameters: its temperature $(T)$ and its number density, largely dominated by the abundance of atomic and/or molecular hydrogen: $n_{0}=n(\mathrm{H})+2 n\left(\mathrm{H}_{2}\right) \mathrm{cm}^{-3}$. The average density of the dust is correlated with the gas density by $n_{d}=10^{-12} n_{0}$. The gas and dust together form either diffuse clouds that are characterized by low density $\left(n_{0}=10-10^{3} \mathrm{~cm}^{-3}\right)$, cold dust $(10-30 \mathrm{~K})$ and hotter gas $(100 \mathrm{~K})$, in which hydrogen is essentially in atomic form, or dense clouds that are characterized by higher densities $\left(n_{0}>10^{4} \mathrm{~cm}^{-3}\right)$, which the dust and gas are cold $(10-30 \mathrm{~K})$ and the hydrogen is mostly molecular. In the dense cold clouds gas-phase species can freeze out on cold grain surfaces $(\sim 10 \mathrm{~K})$ and may chemically react to form new molecules (see Sec. III.C); in this way the formation of polar (i.e., water-rich) and/or apolar (i.e., water-poor) ice mantles occurs. The interaction with photons and cosmic particles drives the formation of other molecules, as has been simulated by experiments performed in several laboratories around the world. Occasional heating in the interstellar medium or the heating induced by star formation inside the cloud lead to segregation, crystallization, and sublimation of ice mantles. Released species contribute to the inventory of the more than 165 chemical species identified in the gas phase. In contrast, only some dozen molecules, the most abundant being water ice, have been identified so far in the solid phase; see Table II. This discrepancy in number is owing to the different sensitivities of radio and infrared techniques; in the gas phase molecules rotate and can be observed with an extremely high sensitivity at radio wavelengths, but in the solid phase the vibrational transitions are detected in the infrared with a much lower sensitivity.

\section{A. Laboratory studies of astrophysical ices}

One way to gain insight into the icy fraction in interstellar medium particles and grains, as well as the composition and physical properties of cometary ice, is to perform laboratory experiments in which models are developed and investigated under circumstances that may resemble, or that would allow extrapolating the achieved results to, the harsher conditions in astrophysical media. Several laboratories have been dedicated to such studies for some time [see, for instance, Ehrenfreund et al. (2001, 2003), Fraser, Collings, and McCoustra (2002), Gerakines et al. (2005), Bernstein, Cruikshank, and Sandford (2006), Muñoz Caro et al. (2006), Hibbitts and Szanyi (2007), Hodyss et al. (2009), and Pat-El et al. (2009), and references therein]. Typical experiments consist of the preparation of ice samples, mainly by vapor deposition of individual substances or mixtures of several components, under controlled conditions of pressure and temperature. The solid fraction may be studied using infrared or Raman spectroscopy, and by transmission (Jenniskens and Blake, 1996a) and scanning (see below) electron microscopy. Similarly, the gas fraction in the chamber may be monitored by mass spectrometry. In some instances, the ices may be exposed to different forms of radiation that may simulate solar or stellar emissions, to study the physico-chemical processes induced in the solid. The heating cycle of comets in their solar approaches is usually simulated by heating the samples, often in temperature programmed desorption experiments, which also provide information on the adsorption and surface interactions of gas and solid phases. 
(a)

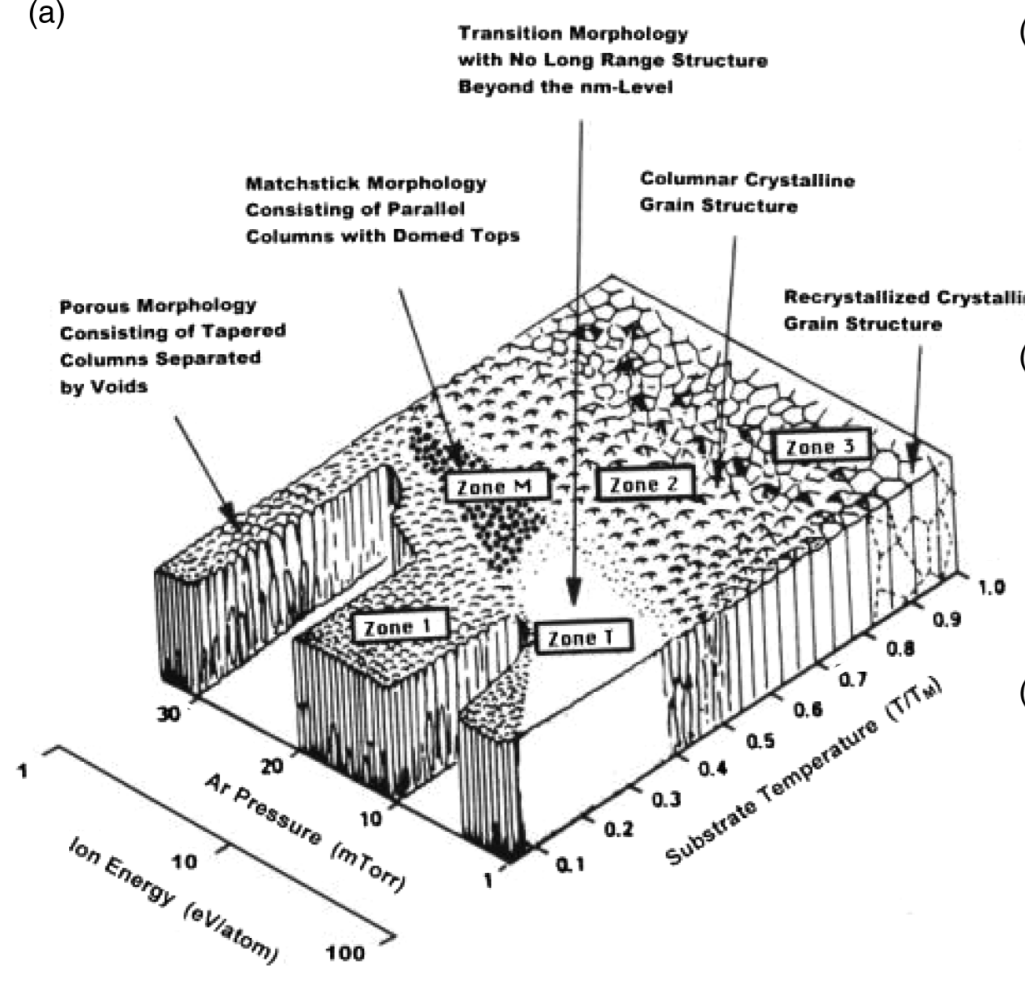

(b)

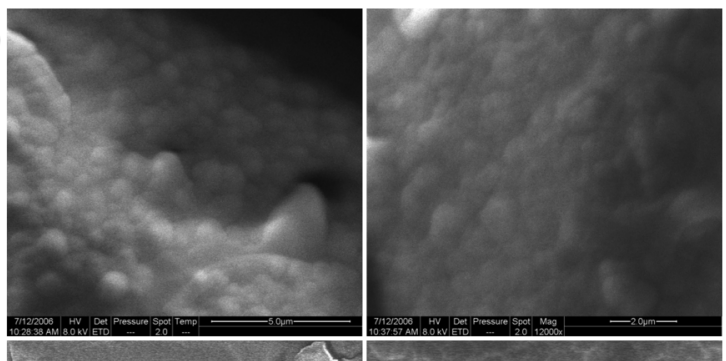

(c)
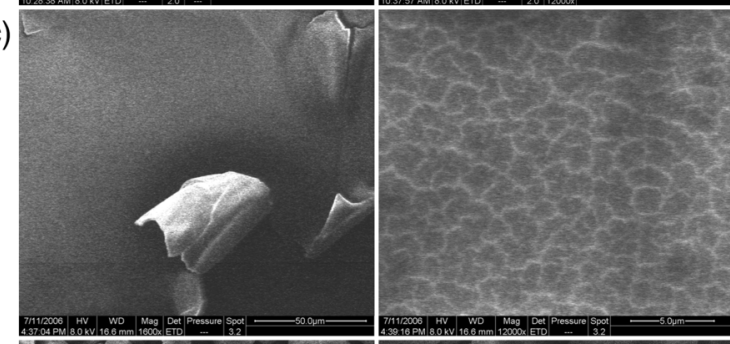

(d)
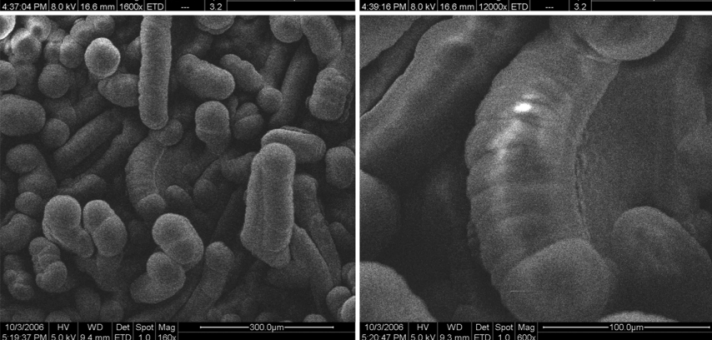

FIG. 9. Film morphologies. (a) A recent version of the structure zone model of solid film deposition (Lakhtakia and Messier, 2005); ice films produced in situ in a liquid-helium cooled ESEM apparatus in water-vapor deposition experiments (Cartwright, Escribano, and SainzDíaz, 2008): (b) zone 1, cauliflower morphology; (c) zone T, transition morphology; (d) zone M, matchstick morphology.

\section{Ice morphology on interstellar grain surfaces}

Dust grains in interstellar space are typically covered with an icy film formed at the very low temperatures of the interstellar medium (Tielens, 2005); it has been estimated that most of the ice in the Universe is to be found on these particles (Jenniskens et al., 1995). Larger icy bodies are supposed to have formed through the accretion and reprocessing of this material (Ehrenfreund et al., 2003; van Dishoeck, 2004). Thus, icy films are found in the interstellar medium, in the Oort cloud, in planetesimals, asteroids, comets, and Kuiper-belt objects, on the surfaces of icy planets and moons, and in planetary ring systems and atmospheres, as mentioned above for the Solar System. Much astrochemistry is presumed to take place on these icy surfaces. A fundamental question, on which hinge many astrophysical and astrochemical processes, is in what forms, and with what morphologies, may such ice films be deposited?

For many years films of numerous different materials, ceramics, semiconductors, and metals, have been deposited onto substrates from the vapor phase. The field is driven by a large number of technological applications, but also has much scientific interest (Lakhtakia and Messier, 2005). One of the key differences between films and bulk materials is in their morphologies, and from the 1960s on efforts have been made to construct a classification of the morphology of a film depending on the conditions of its deposition. This has culminated in the structure zone model. A recent version, reproduced in Fig. 9(a), shows the distinct film morphologies obtained as a function of the renormalized substrate temperature $T / T_{M}$, where $T_{M}$ is the melting point of the material being deposited, and of the ion energy, which is inversely related to the argon gas pressure in the sputtering technique used in that research (Messier, Venugopal, and Sunal, 2000; Lakhtakia and Messier, 2005). The structure zone model describes well the trends seen in experiments with ceramics, semiconductors, and metals. It was recently found that the same morphologies are found in films of ice, despite the very different chemical bonding in ice compared to the materials from which the structure zone model was devised (Cartwright, Escribano, and Sainz-Díaz, 2010).

An ESEM equipped with a liquid-helium cold stage may be used to grow ice films in situ at low pressures and temperatures down to $6 \mathrm{~K}$ appropriate to astrophysical conditions (Cartwright, Escribano, and Sainz-Díaz, 2008). The region of lowest substrate temperature in the structure zone model is occupied by zone 1. Zone 1, or cauliflower morphology, consists of competing void-separated tapered columns whose diameters expand with the film depth according to a power law (Lakhtakia and Messier, 2005). The surface resembles a cauliflower, showing self-similarity over a range of scales; see Fig. 9(b). At a higher water-vapor pressure, there is obtained zone $\mathrm{T}$, or transition morphology, in which there is no longrange structure above the nanoscale. Figure 9(c) shows the featureless surface characteristic of zone $\mathrm{T}$ as well as the boundaries of the individual densely packed grains making up the surface, made visible as charge accumulated on the grain boundaries of the poorly conducting ice surface during imaging. In qualitative terms, the morphology of zone 1 is clearly driven by a competitive process of growth of clusters at all scales, leading to a fractal geometry, while greater gas- 
induced mobility of admolecules in zone $\mathrm{T}$ than in zone 1 smooths out the surface. As they occur at the lowest temperatures, both zone 1 and zone T morphologies can be presumed to be composed of a high-density amorphous ice (see Sec. III.B.1). At substrate temperatures above those corresponding to cauliflower morphology (zone 1), and for relatively high gas-induced admolecule mobility there appears zone $\mathrm{M}$, or matchstick morphology. In the example of this morphology reproduced in Fig. 9(d) we see very large columns tens of micrometers in diameter. The columns display the domed tips characteristic of matchstick morphology and also show interesting substructures on both the tip and along their length, presumably from their growth process; their shape is biomimetic, similar to an icy worm. With regard to the temperatures involved for zone $\mathrm{M}$, it should typically be composed of low-density amorphous ice.

We see that structure zone morphologies do appear in ice films. Can this knowledge contribute to understanding the physics of the structure zone model and of solid ice films? The two axes of the structure zone model shown in Fig. 9(a) are the renormalized substrate temperature and the ion energy, which is inversely proportional to the gas pressure in sputtering experiments. On the other hand, the deposition technique employed here is simply evaporation, involving thermal energies $\left(\frac{3}{2} k T<1 \mathrm{eV}\right)$ for the admolecules. However, the energy required to sputter a water molecule is $\sim 0.2 \mathrm{eV}$ (Bukowski et al., 2007), much lower than the 10-30 eV sputtering threshold for ceramics, semiconductors, or metals (Lakhtakia and Messier, 2005). So we should renormalize the scale of the second axis by the sputtering threshold, in the same way as the substrate temperature is renormalized by the melting point for the first axis. It becomes clear then that the physical basis for the two axes is admolecule mobility induced in one case by the temperature of the film itself, and in the other by the impinging admolecules from the vapor. The latter mechanism is related in these experiments to the temperature of the vapor, and to the presence or absence of helium as an auxiliary gas in the chamber that will thermalize with the chamber walls. The different effects of the two sources for admolecule mobility leading to more or less compact structures surely arise because, unlike thermal mobility, admolecule movement induced by bombardment is highly directional. This directionality is quantifiable in terms of the sputtering yield as a function of the angle of incidence of the bombardment, which has a maximum at $45^{\circ}-60^{\circ}$ (Lakhtakia and Messier, 2005). This is an avenue to explore in moving toward a physical understanding of the structure zone model as a consequence of the competition between the spatially disordered deposition of particles on the growing film surface and the ordering effect of activated particle mobility processes. The ESEM technique, considering the $p-T$ range it covers, is applicable also to atmospheric ices. In one way it is even more appropriate as the time scales covered by ESEM, minutes to hours, are very similar to the actual time scale of atmospheric processes. This is not true for many astrophysical processes, which often take place over much longer times (deposition and aging effects, etc.) and can thus be modeled in ESEM experiments only by rescaling time.
A recognition that these mesoscale structures exist, together with a knowledge of their morphologies, ought to aid understanding of fundamental astrophysical and astrochemical processes involving surfaces coated with icy films and should lead to a reconsideration in terms of the structure zone model morphologies of what is at present in astrophysics often placed under the catchall label of ice porosity (Cartwright, Escribano, and Sainz-Díaz, 2008).

\section{Mixed ices}

Ices composed of mixtures of water, carbon dioxide, and methanol are frequently studied as models for the ice envelope of cometary nuclei and other astrophysical objects (Dartois, Demyk et al., 1999; Bernstein, Cruikshank, and Sandford, 2005; Öberg et al., 2007; Malyk et al., 2007). In recent publications dealing with these systems, evidence of two different $\mathrm{CO}_{2}$ structures, characterized by their different infrared spectral signatures, was clearly exposed (Gálvez et al., 2007; Hodyss et al., 2008; Maté et al., 2009). The spectrum of one of the structures is closer to that of pure $\mathrm{CO}_{2}$, whereas in the other the main bands are shifted to lower frequencies and do not present the typical features of solid $\mathrm{CO}_{2}$ similar to the crystal splitting in the $\nu_{2}$ band, or the sharp peaks in the combination band region. These two structures have been referred to as normal or pure and distorted, or $\mathrm{CO}_{2}$-norm and $\mathrm{CO}_{2}$-dist, respectively. Figure 10 presents two examples from Gálvez et al. (2007) and Hodyss et al. (2008) that show the spectral region of the asymmetric $\mathrm{C}$-O stretch $\nu_{3}$ band for the ${ }^{12} \mathrm{C}$ and ${ }^{13} \mathrm{C}$ isotopomers. Whereas $\mathrm{CO}_{2}$-norm is assumed to be formed by small crystallites of $\mathrm{CO}_{2}$ adsorbed

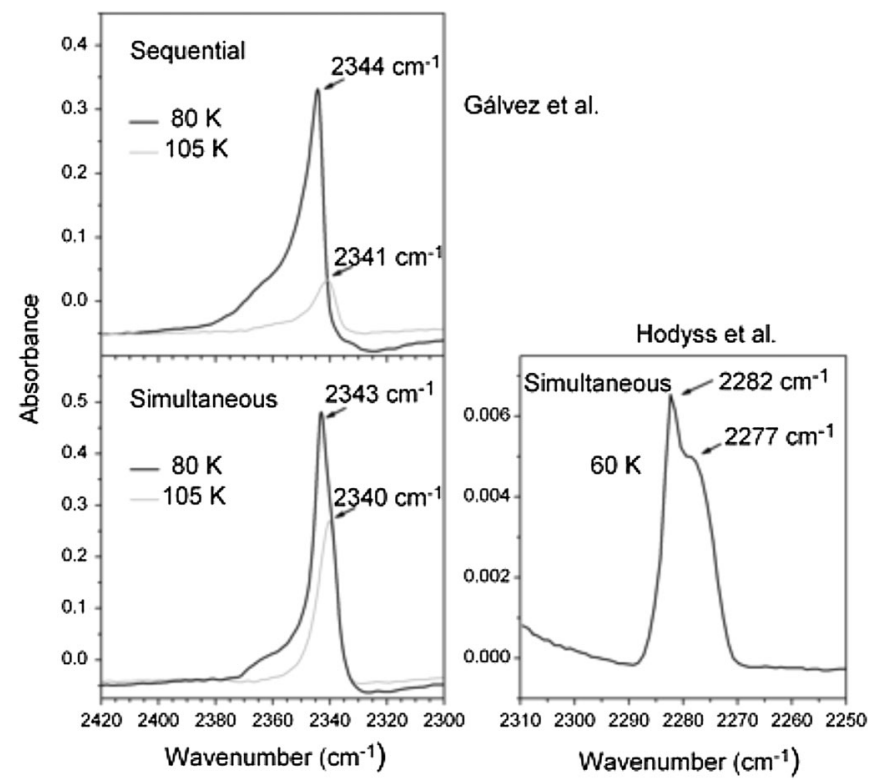

FIG. 10. Transmission IR spectra of mixtures of $\mathrm{CO}_{2}$ and water in the region of the $\nu_{3}$ band of $\mathrm{CO}_{2}$. Left: spectra of two samples obtained by sequential deposition of water and $\mathrm{CO}_{2}$ (above) and simultaneous deposition (below) of the vapors at $80 \mathrm{~K}$. After warming the sample, most $\mathrm{CO}_{2}$-norm has sublimated and $\mathrm{CO}_{2}$-dist is better appreciated. From Gálvez et al., 2007. Right: spectrum focused on the same band for the ${ }^{13} \mathrm{CO}_{2}$ isotopomer. The two $\mathrm{CO}_{2}$ structures are characterized by a band deconvolution analysis. From Hodyss et al., 2008. 
at the surface (in sequentially deposited mixtures) or segregated in larger pores (in simultaneous deposition experiments), the structure of $\mathrm{CO}_{2}$-dist is much less clear. The redshifted vibrations of the latter may indicate some interaction with the surrounding host molecules that weakens the $\mathrm{C}-\mathrm{O}$ bonding in $\mathrm{CO}_{2}$ by $\sim 0.3 \%$. The formation of clathrates retaining $\mathrm{CO}_{2}$ in their interior is not excluded. Although these two $\mathrm{CO}_{2}$ structures are well characterized from the spectroscopic point of view, there are some differences in their behavior when $\mathrm{CO}_{2}$ is mixed with water or methanol. Some of these differences could be explained based on the various porosities of the host ices, but again a full understanding is not yet available. The internal structure of $\mathrm{CO}_{2}$ ices in water and methanol mixtures thus remains an open question in this field. This topic has been further extended in a recent investigation (Cruikshank, 2010). Theoretical calculations of small clusters have been performed (Chaban, Bernstein, and Cruikshank, 2007), but the results are not conclusive, and in some cases seem to point to frequency shifts for the $\mathrm{CO}_{2}$ bands in the opposite sense to the observations. On the other hand, calculations for larger models analogous to solid samples are difficult and have not yet been reported, but they would provide a powerful tool to understand this problem. The eventual spectroscopic observation in astrophysical measurements of the two kinds of $\mathrm{CO}_{2}-\mathrm{H}_{2} \mathrm{O}$ association revealed by these studies would provide a clue to the thermophysical evolution and growth process of the corresponding object. Other results discussed by Öberg et al. (2007), Hodyss et al. (2008), and Cruikshank (2010), which include evidence for the adsorption of $\mathrm{CO}_{2}$ on crystalline water ice under certain conditions (Gálvez et al., 2007), underline the relevance of laboratory experiments on samples containing mixed constituents, as frequently found in astrophysical media.

A special mention should be made of the topic of astrophysical clathrate hydrates, as being a medium to store volatile species within crystalline water cages. Whereas most clathrates of terrestrial interest are formed under highpressure conditions, it was proposed (Buch et al., 2009) that dipolar species such as acetone, formaldehyde, $\mathrm{H}_{2} \mathrm{~S}$, and $\mathrm{HCN}$ can become trapped in water cages at pressures in the 0.1 to $100 \mathrm{~Pa}$ range and temperatures as low as $100 \mathrm{~K}$, and in some cases species such as $\mathrm{CH}_{4}$ or $\mathrm{CO}_{2}$ can also form mixed clathrates with ethers in similar conditions of astronomical relevance. Whereas on Earth most clathrates exist under highpressure conditions, this requirement is not essential on celestial bodies where the temperature is much lower. Dartois and Deboffle (2008) discussed the presence of clathrate species in comets and planets, in conjunction with laboratory experiments aimed to register infrared spectra of $\mathrm{CH}_{4}$ clathrate hydrates. The Cassini mission contributed information that points to a vast reservoir of these hydrates beneath the surface of Titan (Niemann et al., 2010).

\section{B. Cometary ice}

Cometary and trans-Neptunian object (TNO) research constitutes an important topic of Solar System ice studies. Comets and TNOs contain invaluable information on the processes acting during the early stages of Solar System formation. As these objects have a high content of frozen material, the physics of ice is of fundamental importance to extract relevant information from them. In these complex systems, thermal evolution is strongly dependent on structural characteristics, and therefore it is not possible to extract dependable information without appropriate thermophysical models (both theoretical and experimental), including a complete description of both thermal and structural behavior. This is the main problem today in understanding the nature of comets: We do not yet know, for example, either the microstructure or the macrostructure of cometary nuclei and of their constituents.

Cometary nuclei are made up of refractory material (generally described as dust) and a mixture of different frozen volatiles. Water ice is the major constituent, mixed with small quantities of more than 25 other volatiles. The second volatile is $\mathrm{CO}$, with an abundance ranging from $0.01 \%$ up to $10 \%$ that of water, depending on the comet. Other minor volatiles, with an abundance smaller than $1 \%$ of that of water, are $\mathrm{CO}_{2}, \mathrm{NH}_{3}$, and HCN (cf. Table II). One of the most discussed, but yet unsolved, problems regarding comets is the quasisimultaneous sublimation of those volatiles, which have different saturation pressures. If it is accepted that sublimation of volatiles is triggered by insolation, it would be expected that the different volatiles should appear at different heliocentric distances. Nevertheless, it is not possible to find a correspondence from observations between the corresponding temperature of sublimation of the different volatiles and heliocentric distance; see, e.g., Capria (2000). This circumstance suggests that highly volatile constituents, or at least part of them, may be trapped within the water-ice structure, although the mechanisms involved in their trapping and storage are unknown or, at least, controversial. Two different mechanisms have been proposed to describe the trapping of highly volatile molecules: adsorption of volatiles on amorphous water ice and trapping of volatiles in crystalline water ice in the form of clathrate hydrates. There are arguments for and against both mechanisms, and the following illustrates not only the difficulties related to Solar System ice research, but also that progress can only be made with close cooperation among groups with different expertise.

\section{Amorphous versus crystalline ice}

It is thought that comets formed at very low temperatures. This argument is founded, for example, on estimates of spin temperatures of, mainly, water and ammonia [see, e.g., Crovisier (2005), and references therein] which point toward a formation temperature of about $30 \mathrm{~K}$. At that temperature, water ice condenses in an amorphous phase, displaying an open structure and therefore being able to trap other volatile compounds within it. This idea is supported with observations of icy grains in the comae of some comets such as C/2002 C7 and Hale-Bopp. Near-infrared spectra of detected icy grains do not show the characteristic $1.65 \mu \mathrm{m}$ absorption feature of crystalline ice [see, e.g., Davies et al. (1997) and Kawakita et al. (2004)]. Nevertheless, the nondetection of the absorption feature cannot be considered a definitive proof of amorphous ice since that spectral feature depends strongly on the temperature. There are laboratory experiments indicating that initial ice in the grains of the presolar nebula (considered 
seeds of cometary nuclei) is not necessarily amorphous. Moore et al. (1994) performed a series of experiments to characterize spectroscopically water ices condensed on amorphous silicate smokes on an aluminum substrate at temperatures less than $20 \mathrm{~K}$, to simulate the first stages of comet formation. They found that, for smoke particles with a diameter of about 5-10 nm, water ice condensed in a crystalline phase, suffering what they named a low-temperature crystallization (LTC). They suggested that this LTC occurs due to molecular interactions between the condensing gas and reactive silicate surface sites. Leaving the door open to amorphous ice formation, Moore et al. (1994) also mentioned that LTC was not observed for comparatively large grains (of the order of micrometers and larger), suggesting that size of the grains may be an important variable. Later, Schutte (2002) extensively argued that the LTC reported by Moore et al. could be caused by an experimental effect, mainly due to the poor thermal conductivity of the smoke layer. In any case, the most used argument against the existence, or at least, persistence of amorphous ice in comets is based on the results of thermophysical models [see Klinger (1981) and Prialnik, Benkhoff, and Podolak (2003)]. Considering that crystallization is an exothermic transformation, releasing an important quantity of energy, models predict that this energy would trigger a runaway process, producing the crystallization of cometary ice over a comparatively small time scale. Again, this argument has been questioned recently on the basis of laboratory experiments with impure water ice. Kouchi and Sirono (2001) performed experiments of differential thermal analysis on amorphous samples doped with various volatiles and found that crystallization becomes endothermic for $\mathrm{CO}$ "impurities" larger than $3 \%$ by mass, which is a proportion plausible for comets. This result is in line with those of Sandford and Allamandola (1990) and Manca et al. (2001). González et al. (2008) showed that for an endothermic crystallization, amorphous ice could survive for a long time, with a crystallization front comparatively close to the cometary surface.

The crystallization of amorphous ice is a much-studied phenomenon in ice physics. Numerous laboratory studies have been devoted to this topic, e.g., Hage et al. (1994, 1995), Löfgren et al. (1996), Smith et al. (1996), Dohnálek et al. (1999, 2000), Safarik, Meyer, and Mullins (2003), and Safarik and Mullins (2004). All these papers generally agree that the transition is gradual as a function of time and the higher the temperature the faster, leading eventually to cubic ice, ice Ic (see Sec. II.B). The beginning of the transition can be described in the well-known Johnson-Mehl-AvramiKolmogorov approach (Fanfoni and Tomellini, 1998) for nucleation and growth with an activation energy in the range of $70 \mathrm{~kJ} / \mathrm{mol}$ :

$$
f(t)=\frac{\pi}{3} J v^{3} t^{4}
$$

where $f(t)$ is the fraction of transformed phase at a given time $t, J$ is the constant nucleation rate, and $v$ is the growth velocity of the crystal nuclei (N. B., not cometary nuclei). Additionally, see Prialnik, Benkhoff, and Podolak (2003) for details on how crystallization is dealt with in thermophysical models of comets. The work of Jenniskens and Blake (1996a), well known in the astrophysical communities and based on thin-film diffraction experiments, claimed the coexistence of a more relaxed amorphous phase with crystallized ice Ic for temperatures up to $200 \mathrm{~K}$. The existence of this viscous liquidlike or amorphous water is certainly not accepted in the ice-physics community as there is no other evidence for such a mixture of phases; the absorption spectroscopy work by Mitlin and Leung (2002) is quite formal in that the measured spectra can perfectly well be described with optical constants of crystalline ice. Another finding of Jenniskens and Blake (1996a), the release of adsorbed impurities upon crystallization, has been confirmed by later work (Kouchi and Sirono, 2001).

A note on the terminology of amorphous (water) ice in the astrophysical communities is in order here. The multitude of amorphous ices discussed in Sec. II.C has been reduced to a low-density form (LDA) and either one or two higher-density forms, HDA and VHDA; it is unclear at present if HDA and VHDA should be considered as distinct forms or variants of the same form. They are characterized in great detail and LDA comprises older forms such as ASW and HGW; see Sec. II.C. Certainly LDA can be expected to exist under astrophysical conditions, in particular, at temperatures higher than about $60 \mathrm{~K}$. The situation at lower temperatures is more complex. Venkatesh, Rice, and Narten (1974) found evidence for an amorphous form with a density of $\sim 1.2 \mathrm{~g} \mathrm{~cm}^{-3}$ by $\mathrm{x}$-ray diffraction work on samples prepared and measured at $10 \mathrm{~K}$. A more detailed analysis and comparison with similar work at $77 \mathrm{~K}$ was subsequently presented by the same group (Narten, Venkatesh, and Rice, 1976), correcting the density to $\sim 1.1 \mathrm{~g} \mathrm{~cm}^{-3}$ and introducing for the first time the term "high-density amorphous ice," yet still in parallel with the term "low-temperature amorphous ice." Referring to this work, Jenniskens and Blake (1994) took over the term high-density amorphous ice for water ice formed at low temperature, introducing it in the astrophysical communities. There it has stayed ever since, often referred to as "lowtemperature high-density amorphous ice" or $\mathrm{Ia}(\mathrm{h})$. Whether this form is identical to HDA produced at higher pressures and described in Sec. II.C is at present unclear and will be hard to elucidate on the basis of x-ray and electron diffraction data alone. It would be of interest to clarify this situation fully, in particular, as a low-temperature phase transition may well have implications in the astrophysical context [see, e.g., Jenniskens and Blake (1996b)].

\section{Clathrates}

We return now to the mechanisms to describe the trapping of highly volatile molecules within cometary material. Delsemme and Swings (1952) proposed the existence of clathrate hydrates in cometary nuclei to explain the quasisimultaneous sublimation of different ices. This mechanism has been included in several thermophysical models [see, e.g., Houpis, Mendis, and Ip (1985) and Flammer, Mendis, and Houpis (1998)] to describe cometary nuclei evolution. Nevertheless, Klinger (1981) questioned the viability of such mechanisms with thermodynamical arguments, and, today, of the plethora of volatiles detected in comets, the formation of clathrate hydrates under solar nebula conditions has been proven only for $\mathrm{CH}_{3} \mathrm{OH}$ (Blake et al., 1991) and $\mathrm{H}_{2} \mathrm{~S}$ (Richardson, Wooldridge, and Devlin, 1985). Clathrate 
hydrate formation has become an attractive explanation for the trapping of volatiles in recent years, following the visit of comet Hale-Bopp and the analysis of the Stardust mission samples [see, e.g., Brownlee et al. (2006)], which may be consistent with large-scale circulation in the solar nebula. This large-scale circulation suggests that clathrate hydrates could form at comparatively high pressures and temperatures. Gautier and Hersant (2005) proposed clathrate hydrate formation as a plausible mechanism for trapping volatiles that would explain the unexpectedly low value of the upper limit of the $\mathrm{N}_{2}-\mathrm{CO}$ ratio inferred in several long-period comets, as determined by Cochran, Cochran, and Barker (2000) and Cochran (2002). Gautier and Hersant (2005) argued that with $\mathrm{CH}_{4}, \mathrm{CO}$, and $\mathrm{N}_{2}$ being the most abundant species, they consume most of the available water in this order, given their relative affinities for clathrate formation. Since the amount of water is limited, some $\mathrm{CO}$ and $\mathrm{N}_{2}$ molecules may not form clathrates, and they would not be incorporated into at least some cometary nuclei. But this circumstance cannot be used to exclude the possibility of amorphous ice. Laboratory experiments by Notesco and Bar-Nun (1996), work disregarded by the previous authors, show that the underabundance of certain species can also be explained with amorphous water ice, which is more efficient at trapping CO than $\mathrm{N}_{2}$.

Even if, or better, because, a complete picture of cometary ice structure is not yet available, numerical and laboratory experiments on the behavior of mixtures of dusty ices are still necessary, and they have to be designed bearing in mind observational implications. In this regard it is worth mentioning the experiments of Notesco and Bar-Nun (2000) and BarNun, Notesco, and Owen (2007) focused on the effects of clathrate and other gas-trapping mechanisms on cometary ices, those of Gerakines et al. (2005), Bernstein, Cruikshank, and Sandford (2006), and Gálvez et al. (2007), etc., devoted to spectroscopic characterization of ice mixtures, and Trainer et al. (2010), who discuss experiments showing that ethers (for which, however, there is not much evidence at all) may help to speed up the formation kinetics. From a numerical standpoint, a matter pending is to verify whether the equations included in models are able to reproduce the complex patterns of sublimation observed in experiments with ice mixtures.

\section{Heterogeneous chemical processes on interstellar surfaces}

Astrophysicists, particularly those interested in the synthesis of molecules in the interstellar medium, have for several decades recognized the importance of interstellar surface chemistry [see Williams and Herbst (2002)]. Indeed, in the last century, modeling clearly indicated that the formation of the most abundant interstellar molecule, molecular hydrogen, must occur efficiently on the surfaces of the abundant interstellar dust grains (Tielens and Hagen, 1982).

Molecules in the interstellar medium are present in giant interstellar clouds that are cold and tenuous (Williams and Hartquist, 1999; Williams and Herbst, 2002). As mentioned above, such clouds, which are composed of gas and dust, are broadly divided into two classes: diffuse and dense. The conditions in dense clouds allow the dust grains, which are carbonaceous or silicaceous in nature, to accumulate mantles of molecular ices (Williams and Hartquist, 1999; Williams and Herbst, 2002). Spectroscopic surveys show that water, methanol, carbon dioxide, carbon monoxide, and ammonia molecules are often present in these mixed ice mantles (Gibb et al., 2000). Modeling of the chemistry in these interstellar clouds indicates that these ice mantles do not grow solely by the freeze-out of molecules from the gas phase. Heterogeneous processes must also be occurring, synthesizing molecules on grain surfaces which accumulate as ice layers (Oba et al., 2009).

An important question linked to astrophysical ices is the origin of prebiotic material (Meierhenrich, 2008). Muñoz Caro et al. (2002) reported the discovery of amino acids in ice made to mimic cometary ices from $\mathrm{CO}, \mathrm{CO}_{2}, \mathrm{NH}_{3}$, and $\mathrm{H}_{2} \mathrm{O}$; another paper by the same group described the finding of diamino acids in the Murchison meteorite (Meierhenrich et al., 2004). Such diamino acids are the source material for the formation of RNA, which, it is generally believed, made up the first living systems before the appearance of DNA. These works along with others like that of Bernstein (2006) all stress the importance of (inter)planetary and cometary ices as a likely place of formation of such prebiotic material. We discuss this idea further in Sec. VI.B.

\section{Laboratory experiments}

Heterogeneous astrochemistry, as detailed above, is currently of enormous interest in astrophysics. However, there is an urgent demand for data from terrestrial experiments to allow the development of appropriate astrophysical models of molecule and ice formation on interstellar grain surfaces. With regard to the formation of molecular hydrogen, recent experiments showed that this process is possible at low temperatures on a variety of interstellar surfaces (Roser et al., 2002; Vidali et al., 2004; Perets et al., 2005; Creighan, Perry, and Price, 2006; Islam, Latimer, and Price, 2007; Williams et al., 2007; Lemaire et al., 2010). Indeed, on carbonaceous surfaces, it appears that considerable internal energy is present in the nascent $\mathrm{H}_{2}$ (Latimer, Islam, and Price, 2008) and perhaps such excitation may be observable spectroscopically.

Recent results suggest that in the regions of protostars, where the gas is warmer, $\mathrm{H}$ atoms can chemisorb on carbonaceous grains. Pertinent to these regions, elegant scanning probe measurements indicate that $\mathrm{H}_{2}$ formation is rapid on graphitic materials (Hornekaer et al., 2005; Hornekaer, Rauls et al., 2006; Hornekaer, Sljivancanin et al., 2006). With regard to the synthesis of small molecules on interstellar grains, recent work indicated, for example, that methanol can be generated from the reaction of $\mathrm{CO}$ with $\mathrm{H}$ atoms on surfaces at low temperatures (Watanabe et al., 2006).

Much work, however, remains to be performed in the laboratory. Modelers are in urgent need of surface diffusion rates at typical astrophysical temperatures for the accurate prediction of surface reaction rates on water ice and bare grain surfaces (Watanabe et al., 2010). Experiments are required to confirm that $\mathrm{H}_{2} \mathrm{O}$ and other small molecules can be formed via heterogeneous reactions under pseudointerstellar conditions, and some of these experiments have recently been performed (Hiraoka, Mochizuki, and Wada, 2006; 
Hidaka et al., 2009; Mokrane et al., 2009; Cuppen et al., 2010; Dulieu et al., 2010; Ioppolo et al., 2010). Do such reactions generate molecules with sufficient excitation to desorb from the surface, or are the nascent molecules trapped on the surface and immediately incorporated into an ice layer? How are the molecules in the ices processed by the cosmic rays and energetic photons present in the interstellar medium (Thrower et al., 2008; Öberg et al., 2010; Thrower et al., 2010)? What are the morphologies of the water ice and the other ices present in the interstellar gas clouds (Collings, Chen, and McCoustra, 2006; Cuppen and Herbst, 2007; Cartwright, Escribano, and Sainz-Díaz, 2008)? Questions of morphology are particularly pertinent; energized nascent molecules generated in surface reactions on highly porous surfaces are likely to thermalize with the surface before they can desorb (Hornekaer et al., 2003). If heterogeneous molecular synthesis generates a porous ice on a dust grain, how does the structure of the ice respond to the processing by photons and energetic particles that will occur in the interstellar medium?

\section{Desorption of ice-trapped molecules}

Already it seems clear that the desorption of mixed ices, as the dust clouds warm in the vicinity of a newly born star, is more complex than astrophysicists first pictured. Studies showed that the desorption of astrophysically important species from water-rich analogs of interstellar ices does not occur at the single temperature previously assumed in many astrophysical models. The formation of the icy mantles on the interstellar dust grains in the cold $(10-30 \mathrm{~K})$ environment of a dark interstellar cloud involves water and other volatile molecules (e.g., $\mathrm{CO}$ and $\mathrm{CH}_{4}$ ) being trapped on the grain surface. Laboratory experiments show that during the process of stellar evolution, as the dust grains warm, some of the volatile molecules desorb (Bar-Nun et al., 1985; Hudson and Donn, 1991; Ayotte et al., 2001; Collings et al., 2003, 2004; Gálvez et al., 2007; Malyk et al., 2007; Burke and Brown, 2010). However, some volatile species may also diffuse into the pores of the ASW matrix that results from water accretion at the low temperatures of the dense cloud. The structure and significant surface area of the ASW film gives both a range of suitable binding sites for the volatile molecules and a significant capacity for their retention. As the water film warms, the collapse of the pores in the ASW results in the trapping of the volatile species in the water ice. When the temperature of the dust grain increases the ASW may undergo a phase transition to form cubic ice. This transition to cubic ice occurs on time scales that are strongly dependent on the temperature. Upon warming over laboratory time scales, the transition to cubic ice is typically observed at temperatures of $140 \mathrm{~K}$ and above. At lower temperatures $(10-20 \mathrm{~K})$ the time for this recrystallization to form cubic ice is longer than the age of the Universe (Schmitt, Greenberg, and Grim, 1989). This crystallization of ASW upon warming allows the sudden release of the trapped volatile molecules into the gas phase: a so-called volcano desorption (Smith et al., 1997). Further warming eventually desorbs the cubic water ice and any remaining trapped volatiles. These volcano and codesorptions release volatile molecules into the gas phase at higher temperatures than had been assumed in simple models of ice mantle evolution.

An experimental survey shows that the desorption of a variety of different volatile molecules under these interstellar conditions can be broadly classified by the nature of their interaction with the water molecules that make up the ASW host (Collings et al., 2004). For example, "waterlike" species, such as ammonia, bind very strongly to the water matrix and show a single codesorption when the water ice evaporates, whereas "CO-like" species (e.g., $\mathrm{CO}$ and $\mathrm{CH}_{4}$ ), which interact less strongly with the water matrix, exhibit both volcano desorption and codesorption, together with lower temperature "monolayer desorption" from the surface of the water ice. These more accurate desorption data have been incorporated into astronomical models of the evolution of hot cores. Hot cores are the "clumps" of warm gas which are remnants of the collapse of a gas cloud to form a nearby high-mass protostar. Ignition of the protostar warms the surrounding gas and dust initiating desorption of the ice mantles from the dust grains to form the hot core. Recent models of this desorption in the vicinity of a protostar show that correctly describing the desorption of the trapped molecules, and the water ice mantles, allows the gas-phase abundances of the desorbed species to be used as a chemical clock, permitting a determination of the age of the nearby young star (Viti et al., 2004; Williams et al., 2007).

It is also interesting to note that as the dust temperature increases and the ice structure changes, the profile of the infrared (IR) features of the icy species is also modified. This change in the IR profile is considered diagnostic of the temperature of the dust. For example, it has been suggested that the profile of the $\mathrm{CO}_{2}$ ice bands observed in some young stellar objects can be reproduced by using different components having different temperatures (Ioppolo et al., 2009). This is in agreement with the existence of a temperature gradient in the circumstellar environment.

In summary, our understanding of the physics of molecular ices, water and otherwise, and their surface chemistry, under conditions relevant to an interstellar medium, has developed rapidly in recent years. However, a wealth of new data is required from the laboratory to improve astrophysical modeling of the physics and chemistry of this fundamental constituent of interstellar space.

\section{ATMOSPHERIC ICE}

Every crystal was a masterpiece of design and no one design was ever repeated.

\section{Wilson Bentley}

Like snowflakes, the clouds that generate them exhibit what seems to be endless variability. They appear to form under a number of different atmospheric conditions and vary considerably in structures and patterns on relatively short spatial scales. Clouds help to regulate Earth's energy balance by scattering and absorbing radiation and by redistributing heat. They are an essential part of the hydrologic cycle and a prerequisite for life on Earth, delivering the precipitation 
required for rivers to flow and lakes and glaciers to form. Although individual clouds may be short lived, they are an important dynamic variable affecting conditions from the upper atmosphere to the biosphere.

Clouds form when water vapor condenses into liquid water droplets, or directly into ice particles. The droplets or ice particles do not form from water alone; instead solid or liquid aerosol particles provide surfaces on which they may condense. These aerosol particles are omnipresent in the atmosphere and can act as cloud condensation nuclei $(\mathrm{CCN})$ when an air mass is cooled to its saturation point. In order for liquid droplets to nucleate homogeneously from pure water vapor an energy barrier must be overcome. This energy barrier results from the competition between the freeenergy cost of adding a liquid surface $G_{\text {sur }}$ versus the benefit of reducing the bulk free energy of the volume $G_{\text {bulk }}$. This competition is most simply treated using the edifice of classical nucleation theory (CNT):

$$
G_{\mathrm{tot}}=G_{\mathrm{sur}}-G_{\mathrm{bulk}}=4 \pi r^{2} \gamma_{l v}-\frac{4 \pi}{3} r^{3} g_{v}
$$

Here we express the surface-volume competition in terms of Gibbs free energies of a spherical nucleus with radius $r$, surface free energy $\gamma_{l v}$, and free energy per unit volume $g_{v}$. Thus the critical radius of a nucleus is found when $G_{\text {tot }}$ reaches a maximum, or $\partial G_{\text {tot }} / \partial r=0$. This yields a straightforward expression for the energy barrier which must be overcome for nucleation to occur

$$
G_{\text {crit }}=\frac{16 \pi}{3} \frac{\gamma_{l v}^{3}}{g_{v}^{2}}
$$

Previously we used this surface-volume competition in the context of the Gibbs-Thomson effect (see Sec. II.D.1), in order to explain the existence of the vein-node network in bulk ice, which is a thermodynamic consequence of the lower melting point of ice due to the curvature of the individual ice grains. In the atmosphere the same effect, in this context sometimes known as the Kelvin effect, is responsible for a change in vapor pressure over curved surfaces,

$$
p=p_{s} \exp \left(\kappa R_{\text {crit }}\right), \quad \text { where } R_{\text {crit }}=\frac{2 \gamma_{l v} V_{a}}{k_{b} T} .
$$

Here we express the Gibbs-Thomson relation for a spherical droplet of curvature $\kappa=1 / r$ in terms of the vapor pressure $p$ and the saturation vapor pressure $p_{s}$. The critical radius $R_{\text {crit }}$ distinguishes between small droplets that evaporate and larger droplets that can continue to grow and depends on the atomic volume $V_{a}$ and the temperature $T$. As a system cools $R_{\text {crit }}$ decreases, ultimately becoming small enough that droplets can homogeneously nucleate. In CNT, when the energetic barrier to nucleation is overcome, only material diffusion limits the growth of water droplets or ice particles. Thus the steady-state homogeneous volume nucleation rate can be written as

$$
J_{\text {hom }}(T)=J_{o} \exp \left(\frac{G_{\text {diff }}+G_{\text {crit }}}{k_{b} T}\right),
$$

where $J_{o}$ depends weakly on temperature and $G_{\text {diff }}$ is the kinetic barrier to growth. Vehkamäki (2006) is an excellent reference for a complete discussion of CNT.
At cold enough temperatures water droplets may nucleate and freeze essentially simultaneously. In other cases, a second but analogous ice nucleation barrier must be overcome for liquid drops to become solid. In the atmosphere this combination of effects means that in places very high supersaturations can exist and in others micrometer-sized water droplets can be supercooled more than $35 \mathrm{~K}$ before they spontaneously freeze (Pruppacher and Klett, 1997). Soluble impurities and other surface effects may further suppress freezing [cf. Dash, Rempel, and Wettlaufer (2006)], while surfaces of solid particles may promote heterogeneous nucleation at higher temperatures (Cantrell and Heymsfield, 2005).

The majority of cloud formation occurs within the troposphere (see Fig. 11) between the Earth's surface and the tropopause. Within the troposphere surface heating of the boundary layer (typically the first $1000 \mathrm{~m}$ ) causes buoyant moist air to rise, cool, and mix with the dryer air aloft. Internal heat sources and other mechanisms of transport, such as latent heat release, advection, and aerosol adsorption, are also important in influencing this process of rapid mixing. Eventually clouds form as cooling air masses condense. Throughout the troposphere these clouds, depending on their temperature and the local atmospheric composition, may contain ice. Ice and mixed phase clouds are usually formed in cold regions and at high altitudes. Above the troposphere the atmosphere is dry and clouds exist only when very low temperatures prevail. Polar stratospheric clouds (PSCs) form during wintertime when the temperature decreases to $195 \mathrm{~K}$ or lower in the polar regions. The highest clouds form in the polar mesopause region at an altitude of 80 to $90 \mathrm{~km}$, where temperatures may drop to below $140 \mathrm{~K}$ during summertime, making it the coldest place in the atmosphere.

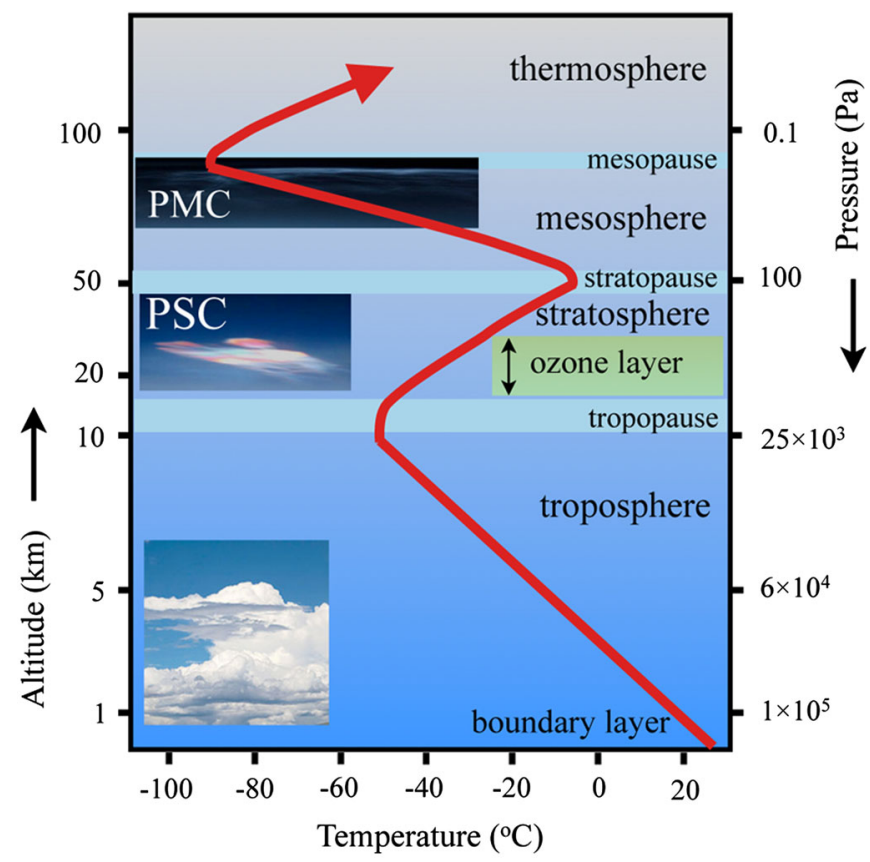

FIG. 11 (color online). A schematic diagram of Earth's atmospheric stratigraphy, depicting the locations of icy cloud layers. 
The development of a detailed understanding of icy clouds in the atmosphere relies on the combined use of field studies, modeling at a multitude of scales, and laboratory studies that provide a microscopic and molecularlevel understanding. Atmospheric ice is studied by remotesensing methods from the ground, and from airplanes and satellites, using passive spectroscopic and light-scattering methods and active methods such as radar and lidar. In the troposphere, and also, with greater difficulty, in the stratosphere, ice is studied in situ using airborne platforms: aircraft and balloons. In situ measurements in the mesopause region are achieved with rocket-borne instrumentation and are limited to brief sampling times as the rockets ascend and descend through cloud layers. These various methods typically lack sufficient access to fundamental physicochemical parameters of ice particles. Furthermore, the representativeness of these types of studies is always an issue because of the transient character of the processes studied. Off-line analysis of collected samples may clarify some aspects, but usually fails for metastable particles or when aging processes are important, because of sample degradation. In these cases laboratory studies may help. Selected experiments can be performed under wellcontrolled conditions to achieve deeper understanding of underlying processes. Theoretical and numerical models are then required to link laboratory and field studies and to transfer this knowledge into large-scale models using sensible parametrizations of key processes.

Here we focus on methods and open questions concerning the microscopic and molecular-level understanding of ice processes in the atmosphere. We begin with a discussion of experimental and theoretical methods used for detailed studies of atmospheric ices, and proceed to discuss the chemistry and microphysics of ice in the mesopause, stratosphere, and troposphere.

\section{A. Measurement and simulation methods}

\section{Experimental techniques}

A multitude of experimental techniques have been developed and adapted to study atmospherically relevant ice processes. Broadly speaking, these techniques focus on specific chemical and physical phenomena using well-constrained laboratory apparatuses. An ensemble of such experiments may serve to simulate atmospherically relevant conditions at a range of scales, from clouds to single particles, and idealized ice and substrate surfaces. The benefit of any experiment can be reinforced if used in connection with field studies and modeling expertise (see Fig. 12). Here we provide a brief overview of the range of experimental techniques applied to atmospheric ice. In Secs. IV.B-IV.D we summarize the results of such experiments and how they apply to ice within different regions of the atmosphere.

Aerosol chambers and flow tubes are considered to be the most realistic laboratory analogs to nature (Callaghan et al., 1994; Stratmann et al., 2004; Khalizov et al., 2006; Möhler et al., 2006, 2008; Hartmann et al., 2011). These relatively large devices contain real aerosol particle ensembles surrounded by gas phases. Although flow-tube designs vary, most seek to input laminar flows of sample aerosols into
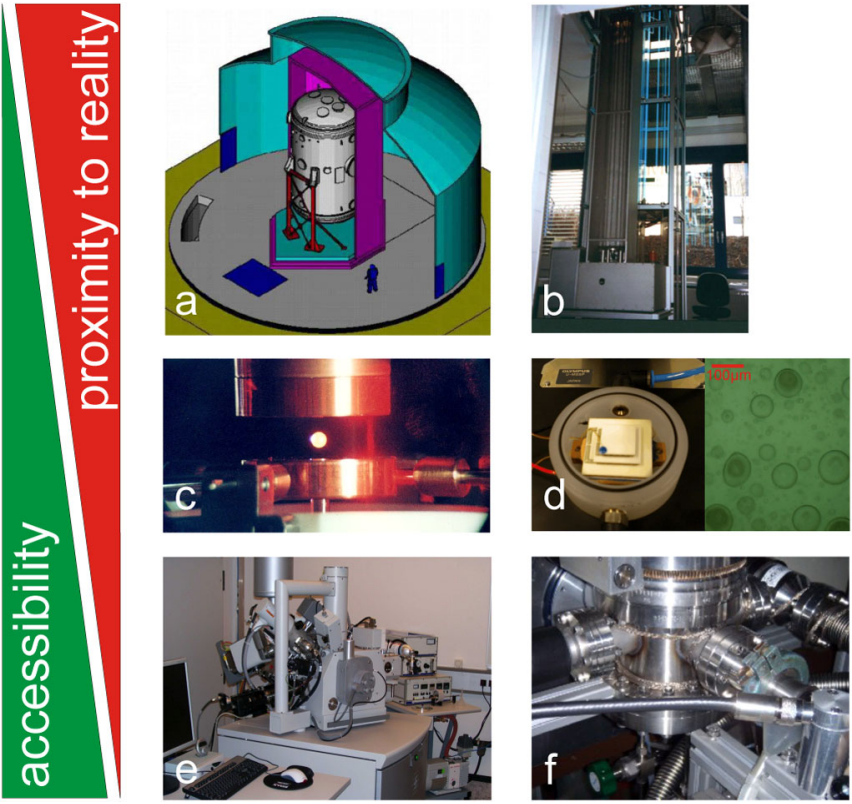

FIG. 12 (color online). Laboratory models sorted by their proximity to the atmospheric reality and the accessibility of physicochemical parameters: (a) aerosol chamber AIDA built for ice nucleation experiments; (b) aerosol laminar flow tube; (c) electrodynamic balance with levitated ice particle; (d) cryochamber with Peltier element suited for light-microscopic observation of the freezing of water droplets in an oil emulsion; (e) ESEM with cryostage and cryotransfer chamber; and (f) UHV cryostat working at $6 \mathrm{~K}$ suited to isolate transient species in rare gas matrices.

chambers with temperature and humidity controlled environments. Often spectroscopic, size-distribution, and chemical analyses are then made using the captured flow [see, e.g., Khalizov et al. (2006)]. The $84 \mathrm{~m}^{3}$ cloud simulation chamber Aerosol Interaction and Dynamics in the Atmosphere (AIDA) is one important example of a large-scale facility whose large volume makes it possible to carry out detailed cloud experiments extending over several hours (Möhler et al., 2006). A representative example is a recent study where the ice nucleation potential of mineral particles was observed to be substantially suppressed if they were coated with secondary organics (Möhler et al., 2008). In related work the ice nucleation ability of mineral dust particles coated with sulphuric acid was investigated at the Leipzig Aerosol Cloud Interaction Simulator (LACIS) (Reitz et al., 2011), which is a thermostated laminar flow tube with a length of up to $10 \mathrm{~m}$ and residence times up to $60 \mathrm{~s}$ (Stratmann et al., 2004; Hartmann et al., 2011).

In the laboratory, macroscopic interfaces, microscopic or nanoscopic particles, and clusters serve as analogs for atmospheric particles. Such investigations are motivated by the idea that laboratory studies of fundamental physicochemical processes can be used to illuminate complex atmospheric processes that are not easily accessible vis-à-vis field measurements (see Fig. 12). Individual laboratory analogs are each somewhat idealistic representations of reality, but can provide access to important parameters. Furthermore, in the laboratory experimental conditions can be precisely chosen allowing sophisticated measurement techniques to be 
applied to outstanding problems. In principle, any strategic research of complicated systems requires such simplifying approaches.

Laboratory-scale experimental techniques that focus on particles include levitation trapping (Shaw and Lamb, 1999; Swanson et al., 1999; Duft and Leisner, 2004; Svensson et al., 2009), powder diffraction (Staykova et al., 2003), and oil-matrix emulsions (Murray, Knopf, and Bertram, 2005). These tabletop-scale experiments allow researchers to focus on the properties of single ice particles or small ensembles of them. Experiments of this type are important for simulating realistic cloudlike environments, where particle interactions and behavior can be investigated. A recent example is highprecision measurements of homogeneous ice nucleation rates in individually charged microdroplets, levitated in an electrodynamic balance, which help to address the question of whether ice nucleation is initiated in the bulk liquid or near the surface of a droplet (Duft and Leisner, 2004). As an alternative, studies may be carried out with a droplet resting on a substrate, which introduces the need to consider the effects of droplet-substrate interactions on the freezing process (Gurganus, Kostinski, and Shaw, 2011).

Still other techniques utilize substrate surfaces as particle surface analogs, typically to investigate fundamental surfacespecific processes. Many modern surface techniques have been adapted from the fields of catalysis and materials science for atmospheric investigations. These include ultrahigh vacuum (UHV) investigations of molecular complexes, clusters, nanoparticles, and adsorbed films (Firanescu et al., 2006). Examples are the use of secondary ion mass spectrometry to investigate interactions between condensed $\mathrm{H}_{2} \mathrm{O}, \mathrm{NH}_{3}$, and $\mathrm{HCOOH}$ molecules (Souda, 2003), molecular beam studies of interactions between water molecules and water ice (Gibson et al., 2011), and the surface-science techniques applied to study molecular-level ice surface properties ( $\mathrm{Li}$ and Somorjai, 2007).

Several surface-science techniques that originally required UHV have also been adapted to higher experimental pressures. Examples include electron microscopy, mass spectroscopy, reflection IR spectroscopy, x-ray photoelectron spectroscopy (XPS), and molecular beam experiments. XPS and electron-yield near-edge X-ray absorption fine structure (NEXAFS) have recently been used to probe ice surfaces in the presence of $\mathrm{HNO}_{3}$ at $230 \mathrm{~K}$ (Křepelová et al., 2010; see also Sec. V.B.1), and molecular beam methods have been used to study ice nucleation in the deposition mode at $215 \mathrm{~K}$ (Kong et al., 2011). These techniques continue to open new pathways for research in atmospheric science, particularly for models of particles in the upper troposphere. Light-scattering techniques are used to determine ice layer thickness (Brown et al., 1996) and surface phase-transition behaviors (Elbaum, Lipson, and Dash, 1993; Thomson, Wettlaufer, and Wilen, 2009). In an exciting new development, laser confocal microscopy combined with differential interference contrast microscopy have been used to visualize the dynamic behavior of individual molecular layers at the air-ice interface (Sazaki et al., 2010). At times multiple techniques, such as light microscopy and molecular beam measurements, can be applied coincidentally (Andersson et al., 2007; Suter, Andersson, and Pettersson, 2007; Lejonthun et al., 2009;
Kong et al., 2011). For example, light reflection measurements can be made simultaneously with molecular beam scattering measurements to constrain adsorbate thickness and fractional coverage on substrate materials (Thomson et al., 2011).

Comparing laboratory investigations of atmospherically relevant ice processes to their real world counterparts is particularly challenging. In the atmosphere, temperature and partial pressures of important trace gases may have quite heterogeneous distributions and are susceptible to constant and sudden changes. Often ice and aerosol particles are not in equilibrium with their environment, resulting in supersaturated gas phases, supercooled droplets, and inherently metastable particles. Such common nonequilibrium conditions make it difficult to identify standard model substances and to compare results between different laboratories and investigators. In many cases essential material parameters such as structure, morphology, surface tension, density, viscosity, and dissociation equilibria vary owing to the nonequilibrium conditions. This variability makes it particularly important for researchers to utilize the full array of laboratory techniques available to them. Repeated measurements of simple systems allow for the most robust comparisons between experimental methods and observed phenomena.

\section{Theoretical methods}

Numerical simulation methods have dramatically improved the molecular-level understanding of many atmospheric phenomena. Similar to experimental design, theoretical calculations strive to simulate reality accurately. Current state-of-the-art molecular-level models of atmospheric ice reaction dynamics utilize the full spectrum of molecular-dynamical, particle-interaction, and turbulence models. Here we summarize the techniques most often used to simulate numerically the microphysical processes associated with atmospherically relevant ice. Such interaction models largely focus on simulating conditions of the upper troposphere and stratosphere due to the global importance of chemical processes such as ozone depletion (Molina et al., 1987; Lowe and MacKenzie, 2008).

MD simulations that use field potentials are often referred to as "classical" MD in chemical physics and are useful tools in ice research. For example, using this class of models, preferential adsorption sites for molecular $\mathrm{HCl}$ on ice surfaces (Buch et al., 2002; Devlin et al., 2002) or the sticking probability of $\mathrm{HCl}$ molecules on ice (Al-Halabi, Kleyn, and Kroes, 1999, 2001) can be simulated. Quantum-mechanical (QM) calculations are also very powerful and can be used for studying minima on potential energy surfaces but are more computationally expensive. The advantages of both classical and quantum-mechanical calculations have been hybridized into techniques called quantum-mechanical molecular mechanics (QMMM). These techniques have been applied by different groups to the problem of $\mathrm{HCl}$ solvation and ionization on and in ice surfaces (Clary and Wang, 1997; Estrin et al., 1997; Svanberg, Pettersson, and Bolton, 2000). Developments of ab initio molecular-dynamics (AIMD) computational techniques allow the dynamical aspect of structures and accompanying properties, such as the infrared spectrum, to be obtained from first-principles calculations 
(Car and Parrinello, 1985). Mundy and Kuo (2006) provided a thorough review of first-principles theory and calculations and their application to atmospherically relevant interfaces.

Solvation and ionization of strong acids such as $\mathrm{HCl}, \mathrm{HNO}_{3}$, and $\mathrm{H}_{2} \mathrm{SO}_{4}$ have become a particularly important field of atmospheric research because they strongly affect ozone depletion. Furthermore, these acids and others are known to be important chemical constituents of clouds in the stratosphere and the troposphere (Zondlo et al., 2000). Although the solvation and ionization of $\mathrm{HCl}$ have been studied theoretically in considerable detail, the same is not true for other strong acids such as $\mathrm{HNO}_{3}$ and $\mathrm{H}_{2} \mathrm{SO}_{4}$. For these compounds some progress has been made using AIMD implementing electronic structure codes (VandeVondele et al., 2005). Balci and UrasAytemiz (2011) studied $\mathrm{HNO}_{3}$ solvation and ionization on and in water clusters and crystalline ice slabs. Using a Kohn-Sham formulation of density-functional theory (DFT) and the Gaussian plane wave method (Goedecker, Teter, and Hutter, 1996) samplings of the Born-Oppenheimer surface were calculated for each time step. This methodology is computationally advantageous, allowing for simulations with thousands of atoms by using first-principles calculations (Mundy and Kuo, 2006). However, as a consequence the simulations use a relatively low level of electronic structure theory and treat the nuclear dynamics classically.

The uptake of $\mathrm{HNO}_{3}$ by ice particles may play an important role for the $\mathrm{NO}_{x}$ chemistry in the upper troposphere and lower stratosphere (Leu and Keyser, 2009), and a detailed understanding of $\mathrm{HNO}_{3}$-ice interactions is required to model $\mathrm{HNO}_{3}$ uptake processes (Kärcher et al., 2009) and to constrain $\mathrm{HNO}_{3}$ redistribution and removal mechanisms (Scheuer et al., 2010). Combined AIMD and DFT methodologies have been used to study single $\mathrm{HNO}_{3}$ molecules interacting with up to eight water molecules. This is a model system for understanding microscopic solvation scenarios of $\mathrm{HNO}_{3}$. Water molecules in the clusters are linked in chains forming either a 2D network with respect to heavy atoms or a 3D network, as shown in Figs. 13(a) and 13(b). Although the chains may not be representative of the condensed phase, the 3D network should be thought of as part of a condensed bulk. A periodic crystalline hexagonal ice slab containing 72 water molecules is illustrated in Fig. 13(c). Different adsorption positions for $\mathrm{HNO}_{3}$ on the surface of the crystalline ice slab may be chosen for calculations run at temperatures below $130 \mathrm{~K}$, and the infrared spectra of the clusters may be obtained from the Fourier transform of the dipole-dipole correlation function. Uras-Aytemiz et al. (2006) and Buch et al. $(2007,2008)$ extended this methodology to other condensed phase systems. An advantage of this approach is that anharmonicities of the system, such as the proton sharing or transfer system, can be ignored because these effects are automatically included.

Numerical simulations of atmospherically relevant ice processes continue to improve as methods become more sophisticated and computation becomes faster. Further advances will be made as the fundamental properties of ice and water (cf. Sec. II) such as density, heat capacity, and the boiling and freezing points are more accurately predicted using numerical models. Numerical simulations are particularly convincing when coupled with validating experimental

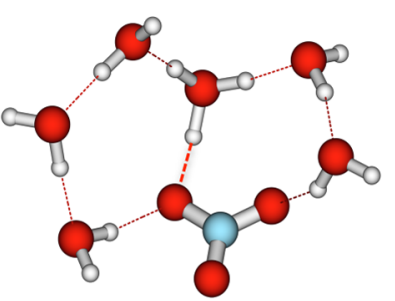

(a)

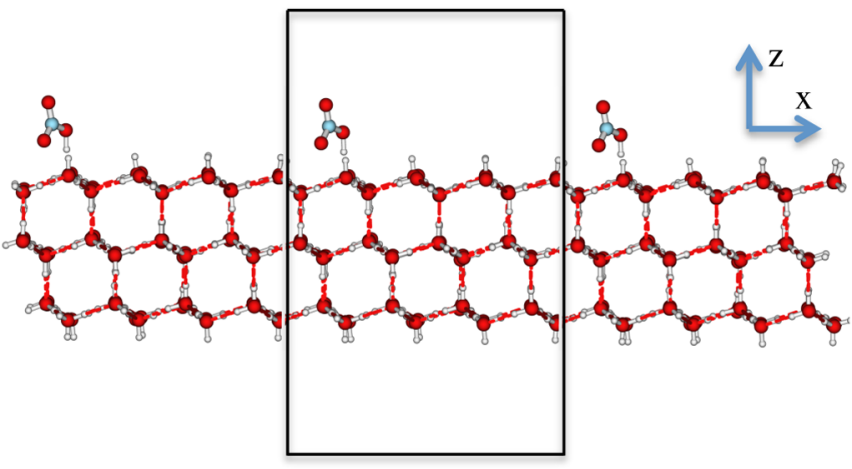

(c)

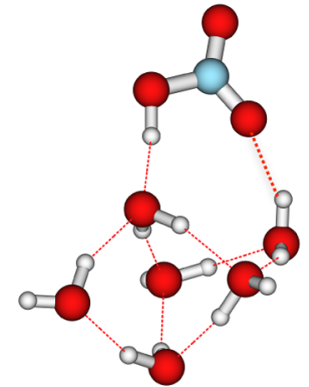

(b)
FIG. 13 (color online). Nitric acid solvation by water molecules: (a), (b) $\mathrm{HNO}_{3}\left(\mathrm{H}_{2} \mathrm{O}\right)_{6}$ clusters in two different configurations and (c) a crystalline cubic ice slab with one $\mathrm{HNO}_{3}$ in the $x$ - $z$ direction.

measurements, and it is only by fusing theory, simulation, and experiment that fundamental physical processes can be illuminated.

\section{B. Ice clouds in the mesopause region}

The highest clouds in the atmosphere form in the polar mesopause region, which can cool to below $140 \mathrm{~K}$ during the summer. The clouds appear as narrow layers at altitudes between 80 and $86 \mathrm{~km}$ and consist of cloud particles with maximum sizes between 50 and $100 \mathrm{~nm}$. Because they become visible to ground observers after dusk when the high-altitude clouds remain sunlit they are known as noctilucent ("night-shining") clouds. When observed from satellite the clouds are known as polar mesospheric clouds (PMCs) (Russell, III et al., 2009). The historical record of their observations goes back to the 1880s, and their presence has been linked to climate change (Fogle and Haurwitz, 1966), although there is ongoing debate on this point (Thomas et al., 2003; von Zahn, 2003). Climate models predict that increasing greenhouse gas emissions should result in cooling of the mesosphere where methane oxidation leads to higher water concentrations, and both effects may contribute to an increased cloudiness (Thomas et al., 1989; Thomas and Olivero, 2001).

The mechanism for ice-particle formation in the mesopause region is not yet fully understood. One hypothesis is that water condenses on existing aerosol particles. Meteoroids that enter the atmosphere vaporize as they reach the thicker atmosphere below an altitude of $120 \mathrm{~km}$ and are visible as meteors from the ground. Vaporized material may 
recondense into nanometer-sized particles and serve as CCN within the mesopause region (Bardeen et al., 2008). Although such dust particles have not yet been proven to exist, they are at present a likely hypothesis for the outcome of meteoroid vaporization, and modeling studies suggest that condensation on dust particles is likely (Rapp and Thomas, 2006). A recent study suggests that negatively charged dust particles could play a significant role in the nucleation process (Gumbel and Megner, 2009). A second hypothesis is that cloud particles are formed by ion-induced nucleation (Sugiyama, 1994). In the sunlit upper atmosphere, photoionization generates $\mathrm{NO}^{+}$and $\mathrm{O}_{2}^{+}$ions. If $\mathrm{H}_{2} \mathrm{O}$ molecules condense on the ions and, by intracluster reactions, form a population of $\mathrm{H}^{+}\left(\mathrm{H}_{2} \mathrm{O}\right)_{n}$, under favorable conditions these may grow into visible ice particles. Still other recent work turns these arguments on their heads, returning to the idea that water may simply nucleate homogeneously under the conditions prevailing in the cold mesopause (Murray and Jensen, 2010). Clearly, the interactions between water, dust, and ionic particles under the prevailing mesospheric conditions of low temperatures and pressures must continue to be studied.

Further complicating our understanding of PMCs is that the ice particles in the mesopause exist in a weak plasma and are influenced by charged particles in the surrounding gas. This may influence any formation process in a significant way. The evaluation of the ion-induced nucleation mechanism requires detailed knowledge of a large number of individual reaction steps that are required to model the formation of cloud particles, some of which are difficult to study experimentally. One example is the dissociative recombination (DR) process in which free electrons combine with water cluster ions (Någård et al., 2002; Öjekull et al., 2007, 2008; Thomas et al., 2010). The neutralization process leads to energy release and partial or complete disintegration of the cluster, a process that competes with particle growth and may hinder the formation of large ice particles. It has only recently been possible to study the process in detail by applying methods usually used in accelerator physics. Absolute rate coefficients for the DR process and the products of $\mathrm{H}^{+}\left(\mathrm{H}_{2} \mathrm{O}\right) n(n=1-6)$ formed during $\mathrm{DR}$ have been determined by the use of a heavy-ion storage ring (Öjekull et al., 2007). Those results help to set stricter limits on models of ice-particle formation in the mesopause region.

Other open questions relate to the morphology and structure of the PMC particles. In particular, what is the molecular structure of PMC particles? It is possible, but unlikely, that they are amorphous, owing to their slow growth over tens of hours by water deposition and rare particle-particle collisions. However, as discussed in Sec. II.C, ice is only slowly transformed from amorphous to crystalline ice around and below 130 K. Surface-specific effects may also play an important role as the particles grow from molecular scales to diameters of tens of nanometers.

Under mesospheric conditions particle-particle collisions take place only rarely, raising a number of questions regarding particle growth and aggregation. Will particles formed by collisions merge into spherical particles? On what time scales do nanoparticles reorganize internally, and how is their shape influenced by further condensation of water? What is the effect of impurity components that may be incorporated into the growing ice particles? These include a range of metals that are found within meteoroids.

Evidence suggests that gas uptake and heterogeneous processes on the surface of ice particles change the mesopause region's chemical composition. Measurements showed sodium is depleted when clouds are present (Thayer and Pan, 2006). The same effect has also been observed for atomic oxygen (Gumbel and Witt, 1998), and model studies indicate that ice particles act as a source for odd hydrogen species that react with atomic oxygen and reduce the concentrations (Murray and Plane, 2005; Kulikov et al., 2010).

\section{Polar stratospheric clouds}

Lower in the atmosphere, PSCs are formed during the wintertime when the air at an altitude of $15-25 \mathrm{~km}$ cools below $195 \mathrm{~K}$. Polar stratospheric clouds are crucial to our understanding of polar ozone destruction. They allow for ozone depletion through denitrification of the stratosphere, and through the catalytic production of active chlorine species that subsequently attack the ozone in the early polar spring (Hanson and Ravishankara, 1991, 1992; Leu and Keyser, 2009).

PSC particles consist of binary and ternary mixtures, mainly of $\mathrm{H}_{2} \mathrm{SO}_{4}, \mathrm{HNO}_{3}$, and $\mathrm{H}_{2} \mathrm{O}$, but thorough analyses of their chemical compositions are difficult to perform (Lund Myhre et al., 2005). Furthermore, the exact nucleation mechanisms of PSCs are still unknown (Tolbert and Toon, 2001). In general it is uncertain which hydrates are in fact present in the aerosols. Several attempts have been made to perform in situ analyses of the chemical composition, phase composition, and the surface structure of PSC particles, leading in some cases to contradictory results (Toon and Tolbert, 1995; Voigt et al., 2000). The metastability of particles causes particular problems for such measurements and the exact composition of supercooled liquids and metastable crystalline particles remains to be determined. In order to assign chemical spectra, precise optical indices of the compounds and phases in question are needed. However, the morphology, density, and dissociation equilibria are also important parameters for convincingly interpreting the gathered spectroscopic information.

In the stratosphere, acids $\left(\mathrm{HNO}_{3}, \mathrm{H}_{2} \mathrm{SO}_{4}\right.$, and $\left.\mathrm{HCl}\right)$ form an array of hydrates and might undergo solid-solid phase transitions (Martin, 2000). The related solids are polymorphs that exhibit a characteristic number of modifications. However, solid-solid phase changes have never been observed in situ in field experiments. The only hydrate determined so far is the nitric acid trihydrate (NAT) analyzed by space-borne infrared limb emission measurements of the Michelson Interferometer for Passive Atmospheric Sounding (MIPAS) on EnviSat (Höpfner et al., 2006). NAT exhibits a high- $(\beta)$ and a low-temperature $(\alpha)$ modification. The latter is metastable and is known only from laboratory experiments. The same is true also both for metastable modifications of nitric acid dihydrate and for cubic ice (Ic). The nucleation rates (Koop et al., 2000), phase transitions (Tizek, Grothe, and Knözinger, 2004), transition kinetics (Grothe et al., 2006), and morphologies (Grothe, 2008) of these hydrates have primarily been studied in laboratories and the results are 
used in PSC microphysical models. Direct observation in the field is still hampered by the lack of suitable detection techniques. In principle, X-ray diffraction would be the method of choice but it is unsuitable in the field due to the sampling geometry. Mid-IR spectra have already proven to provide important results for understanding ice-cloud compositions. However, the interpretation of the spectra depends on high-quality reference spectra from the laboratory and has to take into account certain interlinked effects such as sample texture (orientation), sample thickness, crystal morphologies, and phase composition. In principle, low-frequency Raman and/or terahertz spectroscopy for observing lattice mode vibrations would be the most appropriate methods. Recently, low-frequency Raman data have been measured in the laboratory (Grothe, Lund Myhre, and Nielsen, 2006; Escribano et al., 2007), but far-infrared spectroscopy data, i.e., the $\mathrm{THz}$ range, are still missing. $\mathrm{THz}$ observations are impossible from the ground due to water-vapor absorption lines, but might be obtainable directly in the upper atmosphere or from space. New missions are being deployed with the observation and analysis of PSCs, and also of PMCs, as part of their scientific objectives (Kadosaki et al., 2010).

A specific PSC problem relates to the dissociation of acids in complexes with few water molecules, in environments that could be found in mixed icy particles. This is particularly important for sulfuric acid and nitric acid, which are believed to nucleate homogeneously from the gas phase (Givan et al., 2002; Rozenberg and Loewenschuss, 2009) and potentially include additional trace gases in the nucleation process (Laaksonen, Talanquer, and Oxtoby, 1995). Just how many water molecules are necessary to facilitate the dissociation of an acid? Several experiments and calculations have been carried out to determine whether a few molecules or larger clusters and condensed phases are required. These include molecular simulations of the nucleation process of sulfuric acid (Kurdi and Ponche, 1989; Hale and Kathmann, 1996; Kusaka, Wang, and Seinfeld, 1996). Arstila, Laasonen, and Laaksonen (1998) presented a density-functional study of sulfuric acid hydrate complexes containing 1 to 3 water molecules and were able to show that the proton transfer reaction is unlikely to occur for the monohydrate and dihydrate complexes. For the trihydrate complex the energy barrier is rather low. However, despite these theoretical efforts, proton transfer has not been observed and the mechanisms of solvation and ionization of sulfuric acid, and for nitric acid too, remain unclear owing to proton tunneling effects and the complexity of solution-phase reactions.

Recent AIMD simulations provide a detailed description of $\mathrm{HNO}_{3}$ interactions with water ice (Balci and Uras-Aytemiz, 2011), but the influence of temperature and related effects on the dynamics of the system remain to be determined. Although the different hydrates of $\mathrm{HNO}_{3}$ have been studied experimentally, computational studies are scarce. The hydrates themselves are interesting systems for exploring the dynamics of ions in crystalline environments. Furthermore, most theoretical studies of ice surface chemistry in PSCs concern the water-ice surface, and supplementary work should address the corresponding processes on NAT surfaces, which by comparison are poorly studied (Mantz et al., 2002). Because the hydrates of $\mathrm{HNO}_{3}$ are dominated by ions
$\left(\mathrm{NO}_{3}^{-}\right.$and $\mathrm{H}_{3} \mathrm{O}^{+}$) first-principles molecular-dynamics simulations should be suitable to attack this problem. In addition, there is a general lack of information about ternary systems, which are likely to be present in atmospheric ices. One interesting question concerns the interactions of coadsorbed $\mathrm{HNO}_{3}$ and $\mathrm{HCl}$ on ice. Recent theoretical studies on $\mathrm{HNO}_{3}-$ $\mathrm{HCl}-\mathrm{H}_{2} \mathrm{O}$ clusters (Gómez, Gálvez, and Escribano, 2009; Gómez et al., 2010) could provide the seed to investigate systems of greater complexity leading into amorphous solids.

Although our understanding of PSC particle microphysics and chemistry is improving, the empirical relationships currently used to describe ice-particle microphysics and heterogeneous chemistry in models are based on incomplete data for one or more reaction steps. This drawback, combined with the uncertainties in atmospheric dynamics, causes the modeling of the propagation of PSC effects at global scales to remain of limited accuracy, in spite of the improvements implemented in the models (Kirner et al., 2010).

\section{Ice-containing clouds in the troposphere}

\section{Ice morphology}

The size and shape of tropospheric ice crystals (cloud particles and snowflakes) vary with atmospheric conditions, and in turn the ice morphology has a strong impact on its geophysical effects, from radiative properties to its tendency to agglomerate. As demonstrated by Fig. 14, crystals can have clean geometrical shapes such as hexagonal plates and columns, or more complex geometrical shapes similar to bullet rosettes and dendrites, or fractal-like configurations (Lynch et al., 2002). Nakaya (1954) systematically mapped out the complex dependence of ice-crystal habit as it varies with undercooling temperature and supersaturation [see Ball (2004) for a good summary of the history of snowflake research]. Plates and dendritic snowflake crystals are observed between 253 and $263 \mathrm{~K}$. Below $253 \mathrm{~K}$ habit is dominated by columnar crystals in addition to plates where the particular morphology depends on supersaturation. Above $263 \mathrm{~K}$ crystals form columns or needles before transitioning

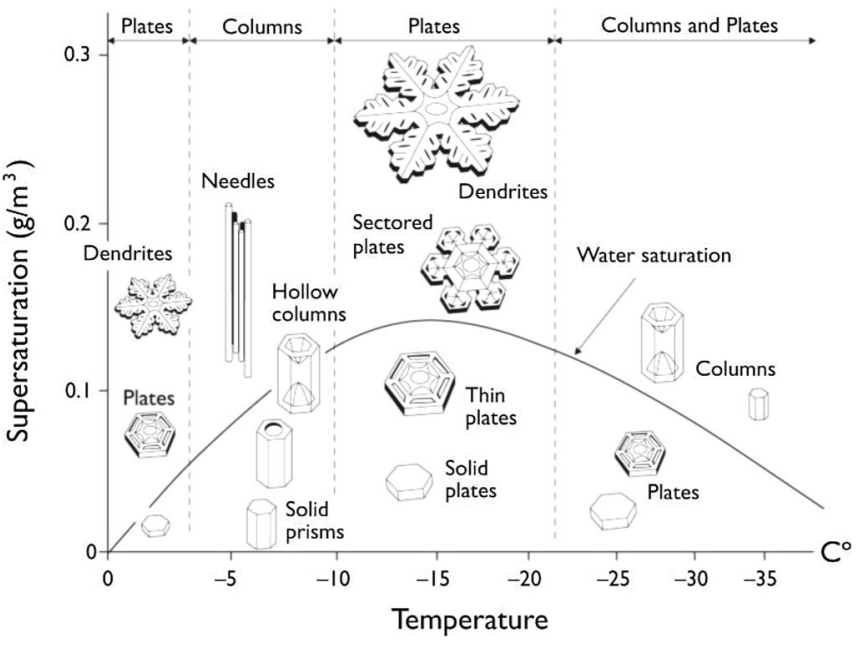

FIG. 14. The Nakaya diagram (Nakaya, 1954) showing snowflake morphology as a function of supersaturation and temperature. From Libbrecht, 2005. 
back to plates and dendrites close to the melting temperature. Field and laboratory studies continue to the present in an attempt to assess more accurately the effect of varying thermodynamic parameters and the process of nucleation [see, e.g., Furukawa and Nada (1997b) and Bailey and Hallett (2004)]. Additionally, the effect of different types of seed aerosols on ice properties has been investigated (Bailey and Hallett, 2002). Field studies suggest that columnar polycrystals dominate the cold upper regions of cirrus clouds, while planar ice crystals are more common in the lower warmer regions (Heymsfield and Platt, 1984; Noel et al., 2002, 2004, 2006). There is also a considerable amount of work being done on the growth of ice crystals from the vapor phase (Libbrecht, 2005).

Although much is known about how ice morphology depends upon thermodynamic parameters, linking this behavior to large-scale processes is often complex. Clouds are dynamic and may contain irregularly shaped particles consisting of faceted polycrystalline particles or smooth sublimating particles, as has been observed in Arctic cirrus and stratiform clouds (Korolev, Isaac, and Hallett, 2000). Icy clouds can be highly inhomogeneous over a range of length scales and regions with high concentrations of small ice particles can be interspersed with regions of large particles (Lawson et al., 2001). Much remains to be learned on the level of individual clouds, including the importance of particle-particle interactions. Such interactions are already known to be significant for thunderstorm electrification (Vonnegut, 1994; Dash and Wettlaufer, 2003; Dash, Rempel, and Wettlaufer, 2006; Saunders, 2008; Cartwright et al., 2011) but may also have other important chemical and physical effects.

\section{Ice nucleation in the troposphere}

Homogeneous freezing of the micrometer-sized droplets formed in the atmosphere usually takes place around $237 \mathrm{~K}$. Although this process has been extensively studied, outstanding questions remain concerning the mechanism for homogeneous nucleation of ice. Laboratory studies of homogeneous nucleation have primarily applied the concepts of CNT, which assumes that nucleation takes place within the volume of the droplet. However, recent studies suggest that even for homogeneous nucleation the presence of surfaces cannot be ignored. In contradiction to CNT, Tabazadeh et al. (2002) suggested that homogeneous nucleation is initiated close to surfaces. There is an ongoing debate on the validity of these mechanisms and the currently available data may not be sufficiently detailed to distinguish between the two hypotheses (Tabazadeh, 2005; Möhler, Bunz, and Stetzer, 2006; Stetzer et al., 2006).

According to CNT the freezing of a solution consists of two consecutive steps: the formation of nuclei and the growth of these nuclei into crystals [see Eq. (7)]. Only if the rates of both are sufficiently high can the respective phase transition occur. If more than one solid phase can nucleate under a set of given conditions, then kinetics rather than thermodynamics controls the phase transition. Thus, the phase with the lowest activation barrier [see Eq. (5)] will dominate the respective nucleation process, depending on the surface free energy and on supersaturation [cf. Tizek, Knözinger, and Grothe (2002)]. This explanation is an example of Ostwald's step rule, which can also be visualized via locally ordered structures in disordered systems: If this short-range order also appears in one of the possible ordered crystalline systems, then this crystallization process will preferentially occur since the surface free energy stays low. This is particularly true for systems containing hydrogen-bonded constituents.

Several types of aerosol particles and dissolved components can act as ice nuclei above the homogeneous freezing temperature. These can initiate heterogeneous freezing, which can be understood with a simple modification to the theoretical treatment of steady-state homogeneous nucleation of Eq. (7),

$$
J_{\text {het }}(T)=n_{s} J_{o} \exp \left(\frac{G_{\text {diff }}+\Phi_{\text {het }} G_{\text {crit }}}{k_{b} T}\right) .
$$

In this description of steady-state heterogeneous nucleation, $0<\Phi_{\text {het }}<1$ quantifies the fractional reduction to the thermodynamic barrier to nucleation due to a change in material properties, external surfaces, etc., that may favor nucleation, and $n_{s}$ is the number density of water molecules in contact with the catalyzing surface. Heterogeneous freezing is traditionally categorized in four ways: deposition (ice deposits directly onto an ice nucleus), condensation (liquid water condenses and subsequently freezes), immersion (a solid particle within a cooling droplet initiates freezing), and contact (surface contact between a liquid and substrate results in freezing) (Cantrell and Heymsfield, 2005). Each freezing mode is active over a typical range of temperature and relative humidity (Meyers, Demott, and Cotton, 1992; DeMott et al., 2010). In particular, contact freezing is active at high heterogeneous freezing temperatures (Pitter and Pruppacher, 1973; von Blohn et al., 2005). The freezing temperatures of evaporating droplets have been elevated up to $4{ }^{\circ} \mathrm{C}$ as immersed solid particles come into contact with the surface (Durant and Shaw, 2005). The contact freezing process has been observed to be even more efficient when aerosol particles approach the liquid surface from the gas phase rather than from within the liquid (Cantrell and Heymsfield, 2005). In another study of contact freezing on a silicon surface covered with different silanes, freezing was observed to occur preferentially at the contact lines where the solid, liquid, and air meet (Suzuki et al., 2007), while a recent study showed no preference for nucleation at the contact line (Gurganus, Kostinski, and Shaw, 2011). Further challenging the simple understanding of phase behavior are new results identifying heterogeneous nucleation from glassy solution droplets (Zobrist et al., 2008; Murray et al., 2010).

For the present, atmospheric ice researchers continue to build predictive fundamental physical models of nucleation processes. A strong lattice match between ice and its substrate may be a good indicator of how effectively a given substrate material promotes ice nucleation, but it is not necessarily predictive (Saunders et al., 2010). Substrate defects that lead to surface charge effects (Park, Moon, and Kang, 2010), the types of bonds formed by adsorbing water molecules, and the types of exposed substrate atoms all affect heterogeneous nucleation. The relative importance of these factors remains an important area of study.

Biological materials serve as particularly interesting tropospheric ice nuclei, especially because they represent an 
important but elusive cryosphere-biosphere interconnection that may be strongly influenced by climate (Pöschl et al., 2010). Airborne terrestrial biological particles such as bacteria, lichen, and fungal spores (Szyrmer and Zawadzki, 1997), pollen (Pummer et al., 2012), and lofted marine species such as diatoms (Knopf et al., 2011; Alpert, Aller, and Knopf, 2011) can play a role in ice nucleation at altitudes of up to 70 km (Imshenetsky, Lysenko, and Kazakov, 1978). However, the specific nucleation mechanisms involved are poorly understood and are complicated by the knowledge that biological materials can just as easily slow (Watanabe and Arai, 1995) or prevent (Pertaya, Celik et al., 2007; Pertaya, Marshall et al., 2007) ice-crystal growth. The actual effect is unique for each type of biological material and can be sensitive to ice surface orientation, structure, and system thermodynamics.

A recent airborne field study analyzed submicrometer icenucleating aerosol particles at an altitude of up to $8 \mathrm{~km}$ using aerosol mass spectroscopy (Pratt et al., 2009). This study found that an important fraction of the ice-crystal residues in cirrus clouds consists of biological material. This surprising result indicates that biological material may play an important role for the radiation budget of the atmosphere and has motivated more detailed investigations. Aerosol-chamber experiments (AIDA) have been carried to investigate the ice nucleation efficiency of biological particles in clouds (Möhler et al., 2007), and laboratory phase-composition studies of simplified systems demonstrated that organic carbon proxies affect the nucleation kinetics (Murray and Bertram, 2008). Continuing studies are required to relate the properties of relevant bacteria, pollen, diatoms, and other biological particles to their ice nucleation activity and to obtain a fundamental understanding of the processes in action.

\section{Cirrus clouds and the supersaturation puzzle}

Ice-containing clouds form in the cold regions of the troposphere, particularly the upper troposphere (UT) as warmer moist air is forced aloft. As previously discussed, ice nucleation may occur either homogeneously from aqueous solution droplets (Koop, 2004) or heterogeneously on aerosol particles (Cantrell and Heymsfield, 2005). Once nucleated, ice particles continue to grow when the watervapor pressure is elevated and excess vapor condenses on existing particles. The resultant decreasing vapor pressure allows the ice supersaturation to relax toward its equilibrium value. In the UT and lower stratosphere (LS), homogeneous freezing is estimated to set in at a supersaturation of approximately 60\% (Koop et al., 2000), while lower supersaturations may be sufficient for heterogeneous nucleation. However, recent field studies suggest that we do not fully understand the conditions under which cloud particles form and grow in the UT (Gao et al., 2004; Lee et al., 2004; Jensen et al., 2005). Excessively large supersaturations have been observed using aircraft, balloon, and satellite measurements (Ekström et al., 2008). Cloud-free regions with persistent supersaturation levels above $100 \%$ have been observed (Jensen et al., 2005) while supersaturations of $30 \%$ have been measured within natural clouds and contrails (Gao et al., 2004). Respectively, these values are above and below typical values we expect for ice nucleation and cloud formation, suggesting our understanding is still incomplete.
How can extreme supersaturations persist in cloud-free regions and why do ice particles not grow according to expectations? Several explanations have been proposed to solve this climatically important "supersaturation puzzle" (Peter et al., 2006). The composition of aerosol particles might inhibit ice nucleation and ice-particle growth. The $60 \%$ homogeneous ice nucleation threshold was established for salt solutions and sulfuric acid, but cloud-chamber data indicate that aerosols containing only organic and elemental carbon may almost completely inhibit ice nucleation (Möhler et al., 2005). Low ice-crystal numbers and high cloud humidity may also result from the presence of glassy aerosols rich in organic compounds (Murray et al., 2010). However, the mechanism of ice nucleation from such glassy droplets remains incompletely understood. Other impurity species such as nitric acid (Gao et al., 2004) and sulfates (Murray and Bertram, 2007) can also affect the growth of ice crystals, and more laboratory studies need to be devoted to their freezing.

Previously, it was assumed that all water molecules colliding with ice crystals would be incorporated into the solid lattice. However, recent laboratory studies suggest that we do not fully understand the basic interactions between water molecules and pure ice surfaces. Laboratory data indicate that less than 10\% (Pratte, van den Bergh, and Rossi, 2006) or even less than 1\% (Magee, Moyle, and Lamb, 2006) of colliding water molecules are trapped by the solid surface. The implication is that most molecules are trapped in a precursor state (Sadtchenko et al., 2004) on the ice surface and desorb before being incorporated into the lattice. Recently, it was shown that surface disorder begins to develop on the topmost layers of ice at temperatures as low as $180 \mathrm{~K}$, and thus ice surfaces are potentially disordered under all conditions prevailing in the UT and LS (Suter, Andersson, and Pettersson, 2006). The effect of this surface disorder on the water accommodation is not currently known.

Experimentally observed equilibrium vapor pressures of supercooled water and ice show variations (Murphy and Koop, 2005) and in situ atmospheric measurements are nontrivial to perform (Peter et al., 2006). However, observed supersaturations are too large to be explained solely by such uncertainties. The kinetically preferred formation of cubic ice (Ic) below $200 \mathrm{~K}$ may partially contribute to the explanation (DeMott et al., 2003; Murray, Knopf, and Bertram, 2005) because the equilibrium vapor pressure for cubic ice is about $10 \%$ higher than that of the more stable hexagonal ice (Shilling et al., 2006). Other explanations may involve the large-scale dynamic development of clouds. Conditions within clouds can vary faster than measurements can resolve, which may cause apparent supersaturation events due to the spatial or temporal averaging (Peter et al., 2006). On the other hand, observations of lower than expected ice-crystal concentrations are in agreement with measured high supersaturations (Krämer et al., 2009). This introduces a "nucleation puzzle," putting a different note on the open questions regarding supersaturation. Clearly, we lack understanding of the details of the freezing process of water at low temperature under atmospheric conditions. Not only it is not clear which type of ice is formed-anything between ice Ih and a fully cubic ice Ic appears to be possible-but the whole process 
leading to the observed low ice-crystal numbers is not fully understood.

The role of Ic, and its effect on supersaturation, is an open problem that deserves specific attention. It has been proposed that snow crystals generally begin with a nucleus of cubic symmetry before they transform into the well-known hexagonal forms (Kobayashi and Kuroda, 1987). In particular, at large undercoolings the Ic form appears to have lower activation energy; i.e., the formation of Ic can be considered a manifestation of Ostwald's step rule. Evidence for Ic in the atmosphere has been claimed from halo observations (Whalley, 1983; Riikonen et al., 2000) but remains inconclusive (Weinheimer and Knight, 1987). However, laboratory observations suggest that, at atmospheric pressures, Ic is crystallized at temperatures below 200-210 K (Kuhs et al., 2004; Murray, Knopf, and Bertram, 2005). There is no indication that this should be different in the atmosphere itself. Rather, the question is: What are the morphologies of Ic formed by homogeneous or heterogeneous nucleation for a wide range of atmospheric conditions (Hansen, Koza, and Kuhs, 2008; Hansen et al., 2008)?

The latter work clearly shows that the formation of ice Ic does not imply a cubic crystal morphology; rather, the expected morphology is trigonal based on the internal crystallographic symmetry of stacking-faulty ice Ic. The rare atmospheric optical phenomenon of Scheiner's halo (Petrenko and Whitworth, 1999) requires ice crystals of octahedral morphologies, thus a truly cubic crystal symmetry. Such crystals may indeed be much less frequent than ice crystals of trigonal shapes observed in cirrus clouds at low temperatures (cf. Heymsfield, 1986). Such trigonal morphologies may well be indicative of the presence of ice Ic, yet this needs to be established more clearly by further investigation.

The interaction of water with aerosol particles and ice particles in the UT and LS is a fairly open issue, which needs continued study due to its climatic importance. The currently available laboratory data and field observations are a good beginning but remain limited. Further laboratory studies and in situ atmospheric observations, combined with better molecular-level modeling of water interactions with surfaces (Krämer et al., 2009), will be key factors for resolving these questions.

\section{Tropospheric chemistry}

In addition to their effects on the radiation budget, ice clouds also influence the chemical composition of the atmosphere. Cloud particles in the stratosphere are now well known to play an important role in the chemistry of the stratosphere. In a similar way, cirrus clouds are likely to influence chemistry in the UT. Solomon et al. (1997) pointed out that the presence of high cirrus clouds near the tropical tropopause could decrease ozone in the stratosphere by activating chlorine constituents. Nitric acid hydrates also form in the troposphere by nucleation on preexisting ice particles (Voigt et al., 2003) or on meteor ablations (Curtius et al., 2005; Voigt et al., 2005).

At lower altitudes, ice chemistry is closely related to the snow chemistry to be discussed in Sec. V.B. Interesting research questions relate to the effects of the quasiliquid layer that exists on ice below the melting point; see Sec. II.D.1 and references therein. Trace gas adsorption and reactions on ice have been treated by Abbatt (2003), and open questions relating to gas uptake on ice and other surfaces have recently been covered by Kolb et al. (2010). Several other important open questions relate to understanding organic chemistry on ice particles and the effects of organic compounds on ice processes (Hallquist et al., 2009). For example, the reversible uptake of small organic compounds on ice has been characterized in flow-tube experiments (Winkler, Holmes, and Crowley, 2002; von Hessberg et al., 2008) and the results have been implemented in models for gas uptake on cirrus cloud particles (Marecal et al., 2010).

The great number of open questions concerning the properties and action of clouds in the atmosphere introduces large uncertainties into models of the Earth's climate system (Lohmann and Feichter, 2005), and our limited scientific understanding is a major problem when we attempt to predict changes in climate over decades and longer. There are urgent needs for further studies that contribute to a detailed understanding of atmospheric ice on the microscopic and molecular levels.

\section{TERRESTRIAL ICE}

The abrupt sides of vast mountains were before me; the icy wall of the glacier overhung me; a few shattered pines were scattered around; and the solemn silence of this glorious presence-chamber of imperial nature was broken only by the brawling waves or the fall of some vast fragment, the thunder sound of the avalanche or the cracking, reverberated along the mountains, of the accumulated ice, which, through the silent working of immutable laws, was ever and anon rent and torn, as if it had been but a plaything in their hands.

\section{Mary Shelley, Frankenstein}

The Earth's surface snow cover strongly affects our lives on a local and on a global scale. It is a subject of great recent scientific interest with respect to its role in global warming, as a fresh-water supply, as an environmental archive, because of avalanches, for its ability to release pollution to the aqueous environment, and owing to its potential to modify the atmospheric composition. In the following we first discuss physical processes related to the above, followed by recent trends in laboratory experiments better to understand the physics and chemistry occurring in or on surface snow. Today, glaciers cover a little less than $10 \%$ of Earth's land surface. Over geological time scales, this amount has varied greatly. Were the ice sheets in Antarctica to melt entirely, global sea level would rise about $57 \mathrm{~m}$ (Vaughan, 2007). At the last glacial maximum, some 18000 years ago, the quantity of water taken up in ice sheets and glaciers meant sea level was about $120 \mathrm{~m}$ lower than today. Most of that change was due to the formation of large ice sheets in northern North America and Europe; however, mountain glaciers, too, had their role. 
Although we do not discuss permafrost, defined as soils with year-round temperatures below $273 \mathrm{~K}$, in detail in this review, it is an important cryospheric phenomenon and the focus of much research. The thawing of permafrost, which can be found over about a quarter of the global land area (French et al., 1996) may lead to the release of carbon into the atmosphere, possibly increasing global warming (Schiermeier, 2001). Thawing permafrost also changes the stability of bedrock, which is of particular importance in mountain areas; for reviews, see Haeberli et al. (2006), Margesin (2008), and Harris et al. (2009). Freeze-thaw cycles in permafrost and in soils that are affected by seasonal frost lead to frost heave and the striking phenomenon of patterned ground, often found in polar and high Alpine environments (Kessler and Werner, 2003; Dash, Rempel, and Wettlaufer, 2006).

The Antarctic and Greenland ice sheets, with $97 \%$ of the glaciated area, or an estimated $99.8 \%$ of the volume, contain the immense majority of the Earth's ice (Ohmura, Wild, and Bengtsson, 1996). Because of their tremendous mass, they react very slowly to environmental changes, with dynamic response times of up to 100000 years for the West Antarctic ice sheet, but at the same time have a great potential to affect sea level (Vaughan, 2007). The much smaller glaciers and ice caps on mountain slopes, also known as alpine, niche, or cirque glaciers, such as the Swiss one that Shelley described, appear to react very sensitively to environmental changes, which makes them, with their widespread distribution over the globe, good candidates for use as climate indicators. In many cases, air temperature, and, in particular, its variation with elevation, has a very high statistical correlation with the mass balance of glaciers (Kaser, 2007); this correlation does not always hold, however, for high-latitude or high-altitude glaciers. Of particular interest with respect to short-term sealevel rise are intermediate-scale icefields and ice caps in Patagonia, Iceland, Alaska, and the Arctic that are small enough to respond to environmental changes on decadal time scales, but large enough to be important with regard to sea-level change (Marshall, 2007).

This direct connection between sea level and glacier volume motivated intense research to understand and predict the fate of glaciers during global warming. In addition, ice and snow act as positive feedbacks in Earth's climate system: The extent of ice cover determines albedo and thus the radiative balance, the height of the ice sheets affects the geometry of upper atmosphere waves, and melt water may influence the global ocean circulation (Andrews, 2007). The most important removal processes of glacial ice are surface melting and ice transport, but it can also take place through sublimation (Kaser, 2007; Marshall, 2007).

\section{A. Glaciers}

\section{Glacier flow}

Glacier flow and consequent ice transport is the main ablation process in cold climates such as Antarctica where almost no surface melting occurs, and constitutes an important process for the Greenland ice sheet (Huybrechts, 2007; Marshall, 2007). The flow of glaciers is a consequence of the weight and creep properties of ice (Schulson and Duval,
2009) and is successfully mathematically described for glaciers that are well coupled to their underlying beds (Marshall, 2007). For these, fluxes are dominated by internal shear stress leading to creep, or to plastic deformation. As ice tends to build up in the glacier, a surface slope is developed. This slope and the weight of the ice induce a shear stress throughout the mass. The two key factors that determine the stress driving the ice flow are ice thickness and the surface slope (Lawson, 2007). Each element of ice deforms according to the magnitude of the shear stress $\tau_{x y}$ at a rate $\varepsilon_{x y}$ determined by the Glen flow law

$$
\varepsilon_{x y}=A \tau_{x y}
$$

In this equation, $A$ is a parameter that is sensitive to temperature, water content, presence of impurities, and crystal properties, factors that also influence the flow velocity (Lawson, 2007). The effect of temperature on $A$, and thus the glacier flow, is well understood, but the sensitivity of $A$ toward the other parameters, especially to the role of impurities, is much more uncertain. Summing or integrating the shear deformation of each element throughout the glacier thickness produces a velocity profile. In this simple case, velocity is approximately proportional to the fourth power of the depth. Therefore, if the thickness of a glacier is only slightly altered by changes in the net mass balance, there will be great changes in the rate of flow. In addition to the above factors, the nature of the bedrock has a great impact on a glacier's flow behavior, and this currently poses the greatest uncertainty in setting the appropriate factor $A$. Erodible bedrock favors rapid ice flow (Lawson, 2007, and references therein) and on hard bedrock, the roughness affects sliding rates. Two mechanisms operate to permit sliding over a rough bed. First, small protuberances on the bed cause stress concentrations in the ice, an increased amount of plastic flow, and ice streams around the protuberances. Second, ice on the upstream side of protuberances is subjected to higher pressure, which lowers the melting temperature and causes some of the ice to melt; on the downstream side the converse is true, and melt water freezes. This process, termed regelation, is controlled by the rate at which heat can be conducted through the bumps. The former process is more efficient with large knobs, and the latter process is more efficient with small bumps. These two processes together produce bed slip. Water-filled cavities may form in the lee of bedrock knobs, further complicating the phenomenon. Although the process of glaciers sliding over bedrock is understood in a general way, more research is needed to account for the nonuniform viscosity of the glacier and the effect of the nature of the bedrock.

Climate scientists have been concerned by the observation of highly accelerated glacier flows, compared to the expectation based on the above considerations, along the margins of the Greenland (Rignot and Kanagaratnam, 2006) and the Antarctic (Pritchard et al., 2009) ice sheets. This acceleration is thought to be a consequence of the retreat of ice shelves that act as barrier for the glaciers and of enhanced lubrication by melt water. The level of understanding of the loss of ice sheets through this accelerated flow is presently too low to allow predictions on its impact on sea level during the $21 \mathrm{st}$ century (Vaughan, 2007). 
To improve the assessment of the terrestrial icefields in the context of global warming, mass balances of ice sheets and glaciers are current foci of research. Remote sensing has significantly contributed to our knowledge of the mass balance of polar ice sheets and has also confirmed much higher than expected mass wastage of glaciers in other regions such as Alaska or Patagonia (Bamber, 2007). Satellite missions with a specific focus on the cryosphere are currently taking place. With these missions, some shortcomings of earlier work should be overcome; they should provide data with greater accuracy and with better coverage of the Antarctic continent.

\section{Glacial ablation}

The physics of snow and ice melt in glaciers and ice sheets is well understood, but owing to the spatial inhomogeneity is difficult to quantify in models (Marshall, 2007). Also, not all melt water runs off a glacier; it can rather refreeze once it percolates into the deeper, and colder, snow pack. This refreezing can lead to a substantial growth of the ice sheet from below; a recent study found that up to half of the entire ice column thickness originated from this source in the Gumburtsev mountain range in Antarctica (Bell et al., 2011). The snow surface receives heat from short-wavelength solar radiation, long-wavelength radiation from clouds or water vapor, turbulent transfer from warm air, conduction upward from warmer lower layers, and the heat released by the condensation of dew or hoarfrost or by the freezing of liquid water. Heat is lost by outgoing long-wavelength radiation, turbulent transfer to colder air, the heat required for the evaporation, sublimation, or melting of ice, and conduction downward to lower layers. Solar radiation is normally the greatest heat source, although much of the incoming radiation is reflected from a snow surface, and most of the heat loss goes to the melting of ice. It is incorrect to think of snow or ice melt as directly related to air temperature; it is the wind structure, the turbulent eddies near the surface, that determines most of the heat transfer from the atmosphere. In glaciers, as in seasonal snow pack, long periods of ablation can lead to $\mathrm{cm}$-scale surface features termed sun cups and penitentes.

Sun cups are characteristic quasiperiodic structures that form on snowfields after extended periods of ablation due to solar radiation exposure. The cups appear as shallow parabolic depressions separated by sharply peaked regions. Qualitatively, these sun cups form because the hollows trap sunlight more efficiently than the peaks. A detailed radiative transfer model of ablating snow surfaces suggested that the size, shape, and time evolution of sun cups can be theoretically constrained using the snow's physical parameters (Tiedje et al., 2006). This work also suggests that unstable sun cup surfaces may lead to the formation of penitentes, another ablation structure found in zones of high sublimation (Post and LaChapelle, 1971).

A penitente is a column of snow, wider at the base and narrowing to a point at the tip, that takes its Spanish name from the resemblance of a field of penitentes to a procession of penitents in white robes. Penitentes commonly form during the summer on glaciers or snowfields at high altitudes; Fig. 15 shows a typical penitente field. The first observations leading

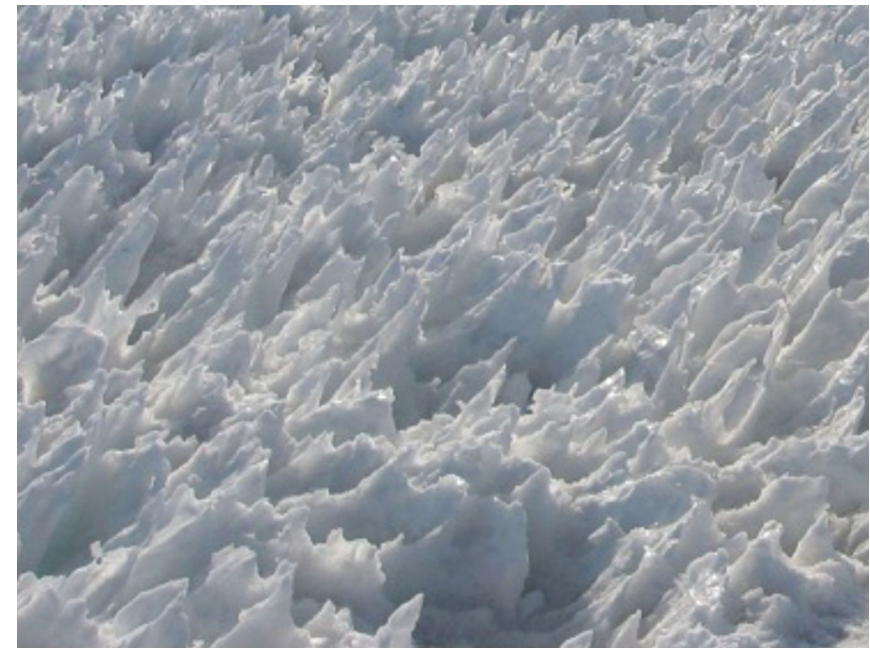

FIG. 15 (color online). Penitentes tens of centimeters long tilt toward the predominant direction of solar radiation.

to the present-day understanding came from Lliboutry (1954), who noted that the key climatic condition for the differential ablation that leads to the formation of penitentes is that the dew point is always below freezing. Thus, snow will sublimate, which requires a greater energy input than melting. Once the process of differential ablation starts, the surface geometry of the evolving penitente provides a positive feedback mechanism, and radiation is trapped by multiple reflections between the walls. The hollows become almost a blackbody for radiation, while decreased wind leads to air saturation, increasing dew-point temperature and the onset of melting. In this way peaks, where mass loss is due only to sublimation, will remain, as well as the steep walls, which intercept only a minimum of solar radiation. In the troughs ablation is enhanced, leading to the downward growth of penitentes. A mathematical model of the process has been developed (Betterton, 2001; Bergeron, Berger, and Betterton, 2006). The effect of penitentes on the energy balance of the snow surface, and therefore their effect on snow melt and water resources, has been described by Corripio (2003) and Corripio and Purves (2005).

\section{Sintering processes}

Glaciers form where the accumulation of snow and ice exceeds ablation. The snow that eventually makes up ice sheets and glaciers undergoes a sequence of conversion stages, which are schematically represented in Fig. 16. Snowfall, in the form of crystals having tiny hexagonal plates, needles, stars, and other intricate shapes (see Sec. IV.D.1), first settles on the ground with a loose granular consistency. As the crystals begin to collapse and break up owing to the weight of the overlying snow, with partial melting and recrystallization the snow reaches a typical density of $0.2-0.3 \mathrm{~g} / \mathrm{cm}^{3}$. These processes proceed more rapidly at temperatures near the melting point and more slowly at colder temperatures and result in a net densification of the snow pack. This snow densification proceeds more slowly after reaching a density of $0.5-0.6 \mathrm{~g} / \mathrm{cm}^{3}$, recrystallization under stress caused by the weight of the overlying snow becomes predominant, and grains change in size and shape in order to 


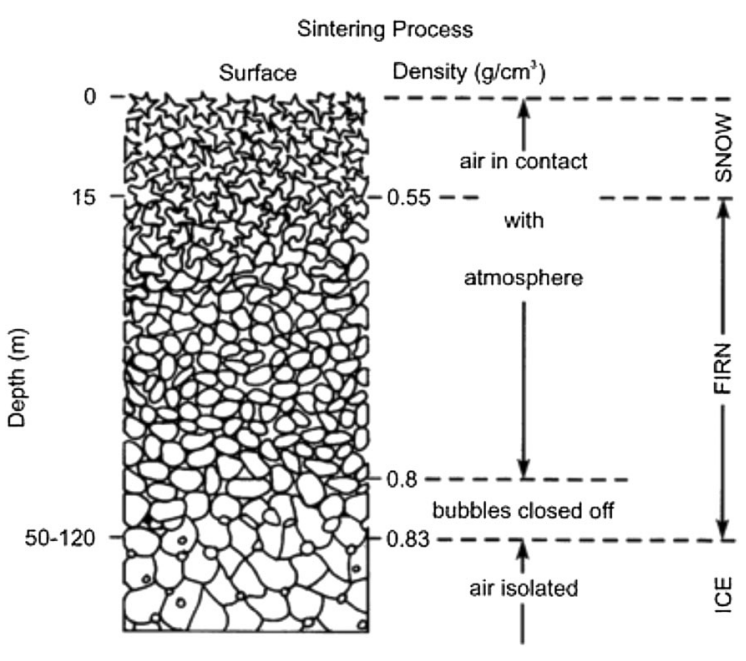

FIG. 16. Schematic cross section of how snow transforms via firn into ice to create glaciers and ice sheets.

minimize the stress on them (see Sec. II.D.1). This change generally implies that large or favorably oriented grains grow at the expense of others. Stresses due to glacier flow may cause further recrystallization. These processes thus lead to an increase in the density of the mass and in the size of the average grain. This stage is reached after one annual thawfreeze cycle, and the resulting snow is referred to as firn or neve and appears as a white structureless mass with far less pore space than a fresh snow pack. Further sintering and fusion occur over time and remaining air is squeezed out or trapped as tiny bubbles. The permeability change at a density of about $0.8 \mathrm{~g} / \mathrm{cm}^{3}$ marks the transition from firn to glacier ice. The transformation may take only three or four years and less than $10 \mathrm{~m}$ of burial in warm and wet environments, but on the high plateaux of Antarctica the same process takes several thousand years and burial to depths of up to $150 \mathrm{~m}$. Most of the air transforms at a depth ranging from several hundred up to over $1000 \mathrm{~m}$ into air clathrate hydrates (Shoji and Langway, 1982) and the ice becomes bubble free; however, rarely does the density exceed $0.9 \mathrm{~g} / \mathrm{cm}^{3}$. An open question is whether grain boundaries or even bulk ice also can take up molecules from the air. The initial densification can be observed by the naked eye as a gradual increase of the clear blue color of old ice along crevasses; while snow is bright white, because of reflection and scattering by the air, ice is blue, because the red part of the optical spectrum is absorbed more efficiently, just as in liquid water.

The consequences of this metamorphism of the ice crystals are far ranging. The direct consequence is a vertical layering of the glacier and, as stress induces sintering, local inhomogeneity of the snow and ice. Both affect the flow dynamics of the glacier, as discussed above. Metamorphism in the snowsurface layer also impacts the chemistry occurring within the snow pack, both directly due to the release of trace gases from the shrinking snow-surface area and because snow properties modify the light penetration through snow (Dominé et al., 2008); see below. This metamorphism, which leads to an overall smoothing of the snow surface, is caused by the higher vapor pressure of small ice crystals making the larger ones thermodynamically more favorable (Ostwald ripening, the
Gibbs-Thomson or Kelvin effect; see Secs. II.D.2 and IV). This process is rather fast; after a day structures of the size of several micrometers can disappear at $233 \mathrm{~K}$ (Legagneux and Domine, 2005; Kerbrat et al., 2008).

When ice cores drilled from ice sheets or glaciers are used as climatic and environmental archives, the depth at which air is totally trapped by glacial ice is of great interest. Before this closure, i.e., in firn, the air is in contact with the atmosphere above, so that a time lag between the age of the ice and the age of the air at any specific depths of the glacier evolves, which depends on the time required for complete trapping of the air bubbles in the glacial ice (Hansen et al., 2007). As the composition both of the enclosed air and of the ice is analyzed and used to reconstruct past environmental conditions, precise knowledge of this time lag over the whole time frame covered by the ice core would be an advantage. This would allow us to understand better the leads and lags of, for example, temperature and greenhouse gases. The physics of the sintering is not well enough known to model this process over time with high-enough accuracy (Loulergue et al., 2007). Recently Lemaire et al. (2010) described a new approach to improve the ice-gas chronologies. In their work, they combine the information of several regional and global markers analyzed in the ice and gas of the ice core with results from glaciological models to establish consistent gas and ice chronologies for several ice cores. The results confirm an overestimation of the gas-ice time lag purely based on firn densification models (Loulergue et al., 2007) and allow the more precise comparison of phase relationships between ice cores from the northern and southern hemispheres (LemieuxDudon et al., 2010).

The layering of snow pack, which is a consequence of metamorphism or sintering, may also foster avalanche formation. One application of current research is to improve our capability to forecast the likelihood of avalanche formation in dry snow potentially triggered by recreationists (Schweizer, 2008). Such dry snow slab avalanches are released when two criteria are met (Schweizer, Jamieson, and Schneebeli, 2003): A weakness of the snow pack below one or more slabs is required and this failure needs to propagate on larger scales. The distinct differences in snow hardness and texture lead to initial failures between two snow slabs. Conditions for fracture propagation are less well known, but snow-pack variability has a strong influence. New three-dimensional imaging techniques are currently being used to visualize snow microstructure with the goal to relate texture to mechanical properties (Pinzer and Schneebeli, 2009). Modeling will then be needed to close the gap between the microscale instabilities and macroscopic avalanche formation. Current modeling includes the sintering process that results in snow failure, but only in a parametrized fashion based on highly idealized structures.

\section{B. Snow chemistry}

Earth's surface snow interacts dynamically with the atmosphere. That the surface snow is thus not simply a passive cover was recognized starting in the 1980s when field measurements above snow-covered polar areas revealed concentrations of a number of atmospheric trace gases that were 
essentially unexpected based on known gas-phase chemistry (Dominé and Shepson, 2002, and references therein). Rapid depletions of ozone and of gaseous mercury up to $1 \mathrm{~km}$ height during polar sunrise, elevated levels of nitrogen oxides, and of oxygenated organics within the snow-pack interstitial air, and emissions of halogens and hydrogen peroxides from the snow are just a few examples of the observed snow-air interactions. The consequences of this reactivity, leading to a modified budget of atmospheric trace gases and of snow composition, are wide, ranging from changing the oxidative capacity and the climate feedbacks of the atmosphere to complicating interpretation of ice-core data as a climatic and environmental archive and fostering the transfer of pollutants to the aqueous phase during snow melt. To identify and to describe quantitatively the individual processes that contribute to the observed air-snow exchange is a current focus of research, a task that has turned out not always to be easy. Here we pick some examples of well-controlled laboratory studies to explain recent developments and to identify some of the open issues. For a broader discussion including recent advances in field studies see Dominé and Shepson (2002), Abbatt (2003), Huthwelker, Ammann, and Peter (2006), Simpson et al. (2007), Grannas et al. (2007), Dominé et al. (2008), and Steffen et al. (2008).

\section{Uptake of trace gases}

Figure 17 illustrates processes that may contribute to changes in snow or in atmospheric composition: physical processes such as the adsorption-desorption equilibrium, the competitive adsorption of several trace gases, and diffusion, as well as (photo)reactions in the snow or dissociation of acidic trace gases. The distribution can further be influenced by dynamic snow processes such as snow metamorphism leading to the release of adsorbed and dissolved species or by the capture of trace gases into growing ice crystals.

For whatever process is governing the overall uptake (emission), the adsorption-desorption equilibrium is the initial (final) step and, as such, has garnered considerable attention during the last decades. It was found that, for a number of oxygenated organics, the interaction with ice surfaces can successfully be described based purely on the adsorptiondesorption equilibrium (Abbatt, 2003). The interest to study the interaction of volatile organics with ice was motivated by their detection throughout the troposphere, where they are an important source of the atmospheric oxidizers $\mathrm{HO}$ and $\mathrm{HO}_{2}$. In these studies the uptake was found to be fully reversible

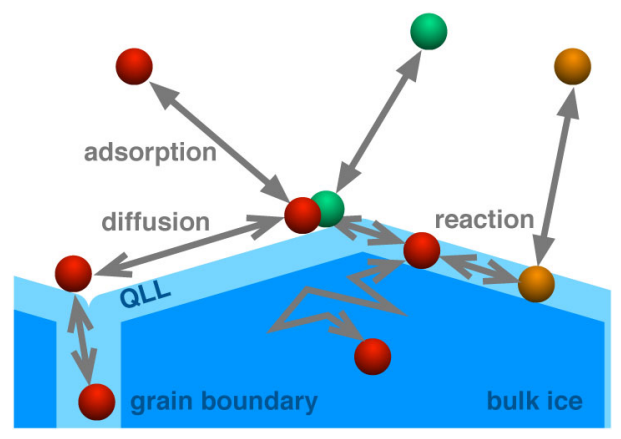

FIG. 17 (color online). Illustration of individual uptake processes. and to increase linearly with rising gas-phase concentration up to a saturation regime when a certain surface coverage is reached. Remarkably, for a wide range of oxygenated organics, the saturation was found to level off at similar surface concentrations of roughly $3 \times 10^{14}$ molecules $\mathrm{cm}^{-2}$. Such an uptake behavior can be well described by the Langmuir adsorption isotherm with its simple linear relationship of the concentration of adsorbed molecules $\left[X_{s}\right]$ and the gasphase concentration $\left[X_{g}\right]$ at low, atmospherically relevant, concentrations:

$$
K_{\operatorname{lin} C}=\frac{\left[X_{s}\right]}{\left[X_{g}\right]} .
$$

The use of the linear relationship is recommended by the International Union of Pure and Applied Chemistry (IUPAC) (Crowley et al., 2010) and is supported by simulations, such as recent MD simulations (Hantal et al., 2008), confirming an uptake process where the organic adsorbs on the ice surface via hydrogen bonds. Knowledge of the temperature dependence of the partitioning allows one to extrapolate to environmental conditions at a specific temperature and snow surface to air volume ratio. There are, however, observations that indicate that under environmental conditions the uptake even of these well-behaved organics is more complicated. For example, Burniston et al. (2007) found in a field study that the observed loss of semivolatile organics from surface snow could not be well described taking into account the adsorption equilibrium and the evolution of the total snowsurface area with time, and concluded that an important factor is missing to describe the emissions, especially for wet snow. In line with this, recent laboratory studies on the uptake of organics at higher temperatures, above some $240 \mathrm{~K}$, showed that the maximum uptake increases well above $3 \times 10^{14}$ molecules $\mathrm{cm}^{-2}$ with temperature (Abbatt et al., 2008). Clearly, there is a need for more experiments in this higher temperature region, most relevant to the Arctic snow pack.

While the laboratory experiments with organics at low temperature showed a fully reversible uptake that can be well described considering purely adsorptive processes, experiments with acidic gases, typically $\mathrm{HNO}_{3}$ and $\mathrm{HCl}$, reveal a more complex behavior. Research interest in $\mathrm{HCl}$-ice interactions has been provoked by the discovery of the stratospheric ozone hole; uptake of $\mathrm{HNO}_{3}$ plays a role both in the stratosphere and in the troposphere (see Sec. IV.A.2). The uptake experiments seem to show two regimes, an initial high uptake followed by a much slower uptake regime with diffusionlike kinetics. Figure 18 compares results from such uptake experiments for HONO and acetic acid, where the gas-phase concentration of the trace gas is shown. This study by Kerbrat et al. (2010) was the first to observe this long-term behavior for the weak acid HONO (nitrous acid), which is an important precursor of the atmosphere's oxidizing agent $\mathrm{OH}$. At $t=0$ the trace gases are exposed to an ice surface leading to a sudden reduction of the gas-phase concentration. After some time the gas-phase concentration of acetic acid recovers just as fast, but for HONO the recovery is initially fast, but then dominated by a slow profile. By focusing on the initial part of the uptake process, information on the adsorptiondesorption equilibrium can be derived. Currently, there are 


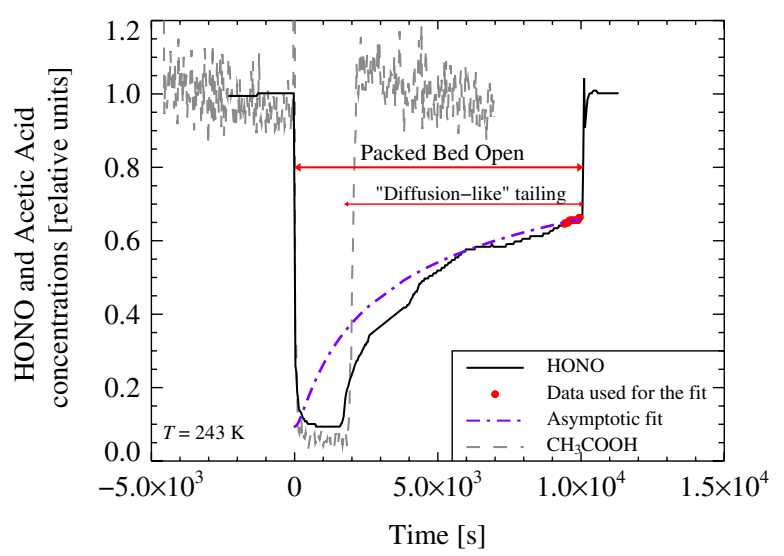

FIG. 18 (color online). Comparison of breakthrough curves observed for nitrous acid (HONO; solid black line) and for acetic acid $\left(\mathrm{CH}_{3} \mathrm{COOH}\right.$; dashed gray line). A packed ice bed flow tube at $243 \mathrm{~K}$ was exposed to the trace gases at $t=0$. For the nitrous acid case, the ice was bypassed at $t=1 \times 10^{4} \mathrm{~s}$. The acetic acid recovered back to its initial concentration before the ice was bypassed, while the nitrous acid trace shows a long-lasting tail. The dash-dotted line represents the result of an asymptotic fit made to retrieve the diffusion parameters that describe the shape of the tail. From Kerbrat et al., 2010.

several procedures used to disentangle the initial adsorptive process from the slower long-term process and a consistent picture of the amount of acidic gases adsorbed to ice surfaces begins now to emerge from some of the more recent laboratory studies (Huthwelker, Ammann, and Peter, 2006). As Table III summarizes, these results, as recommended by IUPAC (Crowley et al., 2010), show a more substantial uptake for acidic gases than that observed for organics at identical partial pressures and temperatures.

The physical nature of the subsequent slow uptake is an essentially open issue. Currently two different approaches to explain the observations are debated: either diffusion into reservoirs such as grain boundaries or slow surface processes such as roughening or restructuring. On the one hand, grain boundaries and junctions occur in natural ice (see Sec. II.D.1; Rempel et al., 2001) and have been shown to host high concentrations of (acidic) impurities (Mulvaney, Wolff, and Oates, 1988); thus it seems reasonable that trace gases might accumulate there after adsorption. Yet, based on this picture, the tail should not be observed on very thin ice films, as the diffusion should be fast enough to penetrate the entire film. Experimental observations of the influence of the thickness of the ice matrix on the observed tail are, however, not conclusive (Huthwelker, Ammann, and Peter, 2006). On the other

TABLE III. Partition coefficient $\left(K_{\mathrm{lin} C}\right)$ of some atmospheric trace gases at $220 \mathrm{~K}$. The partition coefficient gives the concentration at the surface relative to the concentration in the gas phase at equilibrium. Data from Crowley et al. (2010).

\begin{tabular}{lc}
\hline \hline & $K_{\operatorname{lin} C}\left(\mathrm{~cm}^{-1}\right)$ \\
\hline Acetone & $3.5 \times 10^{0}$ \\
$\mathrm{HONO}$ & $3.8 \times 10^{2}$ \\
$\mathrm{HCO}_{3}$ & $8.4 \times 10^{4}$ \\
$\mathrm{HCl}$ & $9.6 \times 10^{3}$ \\
\hline
\end{tabular}

hand, there is strong theoretical and experimental evidence that impurities can influence the surface structure of ice (see Sec. II.D.2; Dash, Rempel, and Wettlaufer, 2006) and, if the kinetics are slow and if this structural change results in a change of the uptake behavior, this might also explain the temporal behavior of the breakthrough curves. Yet, the depth of the restructured regions would need to be quite thick to explain fully the observed kinetics, as noted by Huthwelker, Ammann, and Peter (2006).

There has been much effort spent recently to investigate how impurities impact the surface structure and in turn the uptake behavior. As discussed in detail in Sec. II.D.2, the molecular structure even of clean ice surfaces is disordered at temperatures approaching the melting point. Because some properties of this disordered layer are similar to liquid water, this disorder is often called a quasiliquid layer or, synonymously, a premelted layer. Distinct from this, high concentrations of impurities can lead to (partial) melting of the snow simply due to melting point depression, as observed in brine channels in sea ice. Currently, there are two questions of great importance and scientific interest in this context: How does the presence of the quasiliquid layer impact the interaction with trace gases? And how do impurities at concentrations too low to induce melting impact the surface structure and thus the uptake? Up to now the number of studies that have investigated the uptake of trace gases at temperatures approaching the melting point of ice, where premelting occurs, is limited (Huthwelker, Ammann, and Peter, 2006). Recently, Abbatt et al. (2008) observed a drastic increase in saturation surface coverage at such high temperatures and argued that this is due to a larger uptake to the quasiliquid layer as compared to the solid ice surface. In the most direct way so far, McNeill et al. (2006, 2007) combined results from ellipsometry and from classical uptake experiments to monitor the surface structure of ice. They showed that the long-lasting uptake of $\mathrm{HCl}$ on ice is enhanced when concentrations are high enough to induce surface disorder. Křepelová et al. (2010) used electron-yield NEXAFS spectroscopy to probe ice surface properties in the presence of $\mathrm{HNO}_{3}$ at $230 \mathrm{~K}$. Their results show that nitrate on ice is hydrated by water molecules, just as it is in solution. The spectra further reveal the presence of solid ice at the surface region a few tens of water molecules thick. Likewise, neutron inelastic scattering on ice-covered nanoparticles was recently used to get insight into the water mobility in the ice- $\mathrm{HCl}$ system (Demirdjian et al., 2002). Interestingly, neutron experiments indicated that the translational diffusional mobility of the water molecules sets in at temperatures of some $250 \mathrm{~K}$ (Toubin et al., 2001; Demirdjian et al., 2002), putting in question the existence of a quasiliquid layer at temperatures below $200 \mathrm{~K}$, suggested by McNeill et al. (2007). Clearly, the nature and importance of the long-lasting uptake regime and of changes at the ice surface during adsorption remain a top research priority.

The crucial point is that the temporal behavior, the temperature dependence, the sensitivity to ice morphology, and the reversibility of the uptake are very different in the two uptake regimes, which makes extrapolation of snow-air interactions from laboratory findings to environmental conditions uncertain without precise knowledge of the individual 
processes. For example, at long exposure times the overall uptake is increasingly dominated by diffusive processes and will essentially exceed the adsorptive uptake. Exposure times in laboratory experiments are typically in the seconds to minutes range, while in the environment they may vary from minutes, given by the lifetime of ice in clouds, to days or even weeks when pollutants are exposed to surface snow under stable meteorological conditions. Also, the long-lasting uptake becomes more dominant at higher temperatures, while adsorption increases at lower temperatures, as recently shown by Kerbrat et al. (2010) for HONO. Yet, in a different study using a snow sample of significantly lower density, as found in the environment, no detectable contribution of long-term uptake of HONO to the snow was found, which was attributed to a lower grain-boundary density in the latter sample (Pinzer et al., 2010). This discrepancy underlines the need for a better characterization of ice and snow samples used in the laboratory and found in the field. Another difference of the two uptake regimes is that the initial uptake is mostly irreversible, while the long-lasting uptake has a reversible character, as shown for $\mathrm{HNO}_{3}$ (Ullerstam, Thornberry, and Abbatt, 2005). The irreversibility of the initial uptake calls for a new concept to describe the adsorptive uptake, as the current Langmuir isotherm and the linear relationship rely on equilibrium conditions that are in contradiction to the observed irreversible nature of the uptake process.

In conclusion, experiments at rather low temperatures of $<240 \mathrm{~K}$ or so clearly show that the adsorption-desorption equilibrium plays an important role in the distribution of trace gases between the gas and the snow phase. Extrapolation to environmental conditions is still hampered by insufficient knowledge of the observed long-term uptake and scarce experiments at temperatures approaching the melting point. Whether this distribution will have an impact on a larger scale and thus be of atmospheric significance depends also on the transfer of trace gases between the interstitial, nearsurface, and boundary-layer air and the height of the boundary layer.

\section{Photochemistry of trace gases}

Surface snow hosts a rich photochemistry for two reasons: On the one hand, the high albedo of snow greatly enhances the path length of photons within a snow pack and increases the overall radiation intensity (Grannas et al., 2007); on the other hand, contaminants in the snow pack, such as $\mathrm{H}_{2} \mathrm{O}_{2}$, nitrate, and organics, to name the most dominant, may act as reactive chromophores. In filtered, molten samples from the Arctic and from Antarctica, $\mathrm{H}_{2} \mathrm{O}_{2}$ and nitrate were found to account for about half of sunlight absorption; the other half was attributed to unknown, presumably organic substances (Anastasio and Robles, 2007). Photolysis of $\mathrm{H}_{2} \mathrm{O}_{2}$ is a source of $\mathrm{OH}$ (Chu and Anastasio, 2005), a strong oxidant, for example, of organic matter, possibly contributing to the observed release of volatile organic compounds from surface snow (Grannas, Shepson, and Filley, 2004). Nitrate photolysis is a source of nitrogen oxides that may be released to the atmosphere, where they impact the oxidative capacity (Grannas et al., 2007). In polluted environments this NOx release is of minor importance, but in remote areas it may be the primary source of gas-phase nitrogen oxides. At the South
Pole the emissions of nitrogen oxides have even reached concentrations in the shallow boundary layer high enough for ozone production, an observation normally restricted to polluted environments (Crawford et al., 2001).

The consequences of photochemistry occurring in snow are not restricted to modifications of the atmospheric trace gas budget, but may also alter concentrations within the snow pack. Nitrate content in the surface snow at low-accumulation sites, such as in Antarctica, has been found to be highly influenced by postdepositional processes, one of which, although not the major process, is photochemistry (Blunier et al., 2005). This has consequences for the interpretation of concentration profiles gained from ice cores, where nitrate is an easy ion to measure, as an environmental archive. In the same way, ice cores provide records for reconstructing the mercury content preserved in snow from long before anthropogenic influences began (Steffen et al., 2008). They would thus be a valuable tool to study the natural atmospheric mercury cycle, provided that surface snow loss processes are well understood. Field studies revealed that polar surface snow loses its mercury deposited from the atmosphere, the emission of which is enhanced with solar irradiation. In line with the known mercury chemistry in the (liquid) aqueous phase, Bartels-Rausch et al. (2011) showed that organics can enhance photolytic mercury release also in the ice phase and at low temperatures. Some studies showed photolytic decomposition of anthropogenic organic compounds with products of increased toxic effect (Grannas, Shepson, and Filley, 2004). Furthermore, organic radical compounds, products of irradiation of organic chromophores, can participate in subsequent bimolecular reactions, for example, with mercury (Steffen et al., 2008). This chemistry directly impacts the amount of toxic substances in the snow pack, and thus the amount that can possibly be released and enter the biosphere during snow melting. Field and laboratory studies revealed that snow photochemistry affects the composition both of the boundary-layer air and of the snow pack. Many relevant processes have been identified and a more quantitative picture starts to emerge, but we are far from understanding the fundamental chemistry, which hampers extrapolations to environmental conditions. In the following, we discuss current limitations and research topics.

Products from photochemistry in snow may differ from those found in aqueous environments. For example, unusually high relative yields of HONO and NO are detected, both of which are secondary products of nitrate photolysis. The large temporal and spatial variations of the relative nitrogen oxide emissions found in field measurements underline the complexity of their production and release. Identifying environmentally relevant production processes is a current focus of research and several ideas, such as the reaction of photolytically produced $\mathrm{NO}_{2}$ with organics (Bartels-Rausch et al., 2010) or hydrolysis of $\mathrm{NO}_{2}$ (Zhou et al., 2001) have been proposed. Studies on organic photochemistry in ice also revealed some interesting chemistry: Matykiewiczova, Klanova, and Klan (2007) reported unusual photobehavior of halobenzenes, the photolysis of which produced biphenyls that are more toxic than the educts. The rates of reactions may also be substantially different in ice as compared to in liquid solutions (Grannas et al., 2007). Recent studies showed 
reaction rates accelerated by up to a factor of 40 during the photochemistry of organics (Grannas, Bausch, and Mahanna, 2007; Kahan et al., 2010), but this acceleration is especially important for bimolecular reactions, which often follow a photolysis reaction, such as the oxidation of halogenides and nitrite, where rates of about $10^{5}$ times faster were determined in ice (Takenaka, Ueda, and Maeda, 1992; Grannas et al., 2007).

It is questionable whether results from laboratory studies that focus on identifying photolysis pathways can be directly applied to field studies, as the location and physical state of pollutants might be very different. Little is known about the chemical form and location of the reactive impurities in surface snow: Are they attached via hydrogen bonds on the surface or dissolved in a surface layer or in liquidlike micropockets, grain boundaries, or veins? Do they form supersaturated solid solutions or pockets of brine? Contaminants may enter the snow pack via nuclei of cloud condensation or ice formation, deposited aerosols, and adsorbed trace gases. A current limitation in this respect is that most laboratory studies use ice samples that have much higher contaminant concentrations than those found in natural snow samples. Segregation of solutes from the forming ice during the freezing process may depend on solute concentration and possibly occurs only when a certain solubility limit of the ice is exceeded. So even if wet metamorphism and refreezing occurs in the field, it is not certain whether segregation occurs in nature, as concentrations are so much lower. Experiments that more closely mimic the characteristics of natural snow are required.

The microstructure of ice results in flow and sintering of glacial ice. Many open questions are currently tackled by addressing the microstructure of snow with its vast network of grain boundaries and veins. A better understanding of its properties will help to predict, for example, avalanche formation, the uptake and release of trace gases, and chemistry occurring in the snow pack.

\section{SEA ICE}

Pack ice might be described as a gigantic and interminable jigsaw puzzle devised by nature.

\section{Ernest Shackleton, South!}

A distinctive and crucial feature of water is that the solid, in the form of ice Ih, floats on the liquid, so sea ice generally forms at the ocean-atmosphere interface. Sea ice, ice that originates as frozen ocean water, must be treated as a distinct genus of ice to that on land. It possesses a multiphase structure that, in addition to the ice-crystal lattice, includes also gas, liquid brine, solid salts, and other impurities. Sea ice on the microscale is a so-called mushy layer (Feltham et al., 2006) and on that scale the presence of salt in the sea makes double-diffusive convection of heat and salt an important physical process.

In this section, we focus on morphological features of sea ice, those physical processes that create the landscape of sea ice, and how these processes are taken account in large-scale numerical models. On a geophysical scale, sea ice is considered to be a mixture of open water and several different classes of undeformed and deformed ices. Each ice class has an inherent ice-thickness distribution, but ice classes also differ in their mechanical and thermodynamical properties. A typical floe of sea ice consists of undeformed ice, whose thickness varies from 0.5 to $5 \mathrm{~m}$, and deformed ice or pressure ridges, whose thicknesses range from 2 to $30 \mathrm{~m}$ in the Arctic Ocean.

A typical feature of pack ice is the strong gradient of properties in a vertical space. Pack ice may consist of columnar and platelet ices, upon which may be superimposed through precipitation ice formed as a result of the sintering of snow and pure snow layers. Density, brine content, crystal structure, and prevailing orientation are different for these ice types. The top 1-10 cm of sea ice is usually composed of randomly oriented ice crystals less than $5 \mathrm{~mm}$ in diameter, reflecting turbulent conditions during the initial freezing stage and freedom of growth in the horizontal directions. After this initial ice-cover formation, ice grows downward and ice crystals become larger. Deeper layers of ice are anisotropic because the crystals having the $c$ axis in a horizontal plane are favored to grow larger as heat is removed more efficiently in this orientation. This process ultimately leads to a uniform columnar ice type where the brine is trapped between the ice crystals (Weeks, 2010). The bottom of the ice is porous and constantly interacting with the ocean water beneath.

Traditionally, large-scale sea-ice thickness characteristics have been mapped by profiling either the bottom or the surface of the ice with sonar or laser, which gives information about sea-ice draft or freeboard (see Fig. 19), respectively (Wadhams, 2000). During the last few years, the utilization of side scanning sonars and lasers extended these methods to fully three-dimensional mapping, enabling a much more detailed examination of the morphological characteristics of sea ice (Hvidegaard, Forsberg, and Skourup, 2006; Wadhams and Doble, 2008). Figure 19, based on lidar measurements in the Baltic Sea in March 2005, depicts a highly deformed sea-ice field. In this length scale of $1 \mathrm{~m}-1 \mathrm{~km}$, dominant features are the pressure ridges and other deformed ice types, which can be identified from the image from their high freeboard and narrow elongated shape. At the time of the imaging, the mean ice thickness in the region was about a meter, but by purely thermodynamical means the ice would only have been 20-30 cm thick, which emphasizes the importance of a dynamical thickening of the ice due to deformation.

Right from its beginnings, in and even before Shackleton's time, sea-ice research has been motivated by practical applications: shipping and off-shore activities in polar regions, exigencies of the Cold War, and climate-related questions. As the pack ice of the Arctic Ocean experienced remarkable shrinking and thinning during the last decades, climaterelated investigation has become the most important topic in sea-ice research. The most apparent changes are observed in the average and annual minimum sea-ice extents (Comiso et al., 2008) and in the mean sea-ice thickness (Rothrock, Yu, and Maykut, 1999; Haas et al., 2008); but the drift speed and rate of deformation have also increased (Rampal, Weiss, and Marsan, 2009), and the age and residence time of the sea ice in the Arctic have decreased (Maslanik et al., 2007). 

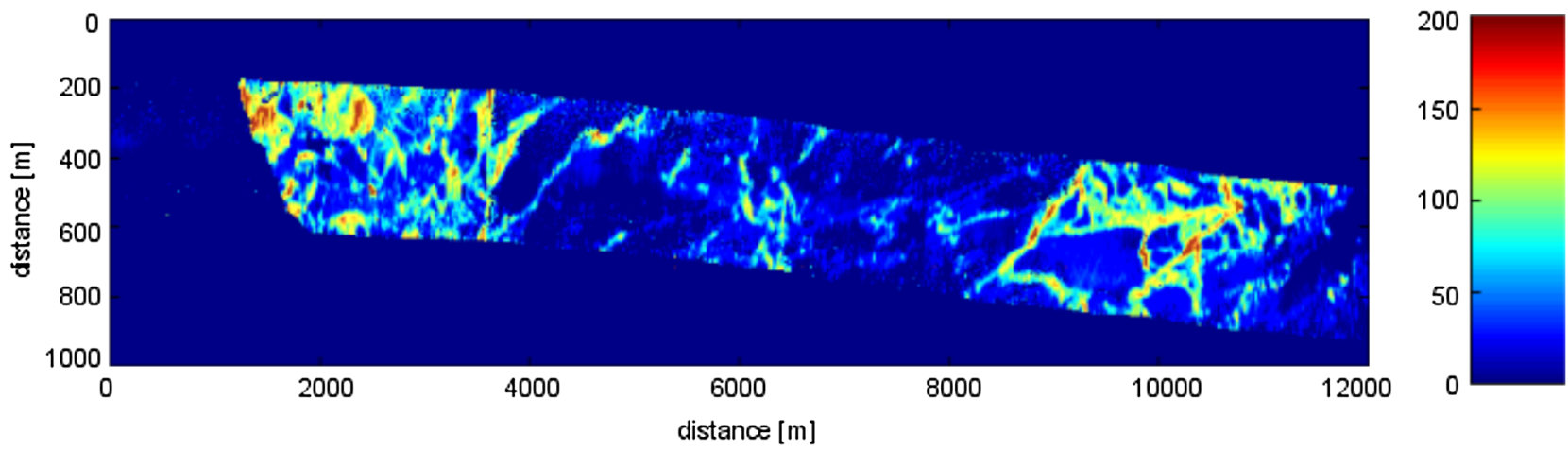

FIG. 19 (color online). Lidar image of sea-ice topography in the Baltic Sea. The width of the swath is $400 \mathrm{~m}$. Colors and shades indicate a freeboard of ice (height of the surface relative to the ocean surface) in centimeters.

All climate models, which prognostically calculate the response of the pack ice to the increase of atmospheric $\mathrm{CO}_{2}$ and other greenhouse gases, predict shrinking and thinning of the ice cover, and ultimately the total disappearance of the perennial ice cover in the Arctic Ocean within the next 30 years (Wang and Overland, 2009). Another result common to different climate models is that the increase of the surface air temperature will be 2 or 3 times larger in the Arctic than the average over the Earth (Holland and Bitz, 2003). Classically, polar amplification of climate change is understood to be caused by the positive feedback mechanism of snow and sea-ice changes, but changes in the atmospheric meridional transport of heat and moisture are also very significant driving factors (Graversen et al., 2008).

Although climate models projected similar changes to those that have occurred, modeled sea-ice changes are much smaller in magnitude than what was observed during the last 5 years (Stroeve et al., 2007). Some climate models project a similar accelerated disappearance of sea ice, but not until 10-20 years from now. It is well known that the natural variability of the climate is very large in the Arctic, which makes it difficult to assess to what extent the present changes are amplified by natural climate variability. In principle, climate models are able to reproduce internal climate variability when multiple simulations for the same climate periods are conducted. However, the ability of an ensemble of climate-model simulations to capture real natural variability is presently limited and thus it is as yet an open question whether the tipping point for accelerated melting has already been reached, or whether the present models are too conservative because of simplifications of some critical processes of ice physics.

\section{A. Processes determining the evolution of pack ice}

Pack-ice characteristics on a scale larger than the typical length scale of individual ridges and floes are described by the ice-thickness distribution function $g(h)$ (Thorndike et al., 1975) (see Fig. 20), defined as

$$
\int_{h_{1}}^{h_{2}} g(h) d h=\frac{1}{R} A\left(h_{1}, h_{2}\right)
$$

where $R$ is the region to be considered and $A\left(h_{1}, h_{2}\right)$ is the area within which the ice thickness varies between $h_{1}$ and $h_{2}$.
The evolution equation for $g(h)$ is $D g(h) / D t=\Theta+\Psi$, where the left-hand side of the equation describes the local change and advection, and $\Theta$ and $\Psi$ are the thermodynamic growth rate and the redistribution of ice thickness due to deformation, respectively.

The engine for sea-ice evolution is heat and momentum exchange between the ocean and atmosphere. Sea-ice dynamics is mainly driven by wind and ocean stresses. The thickness distribution and momentum balance are strongly coupled via the internal stress of pack ice. The material properties of sea ice depend very much on ice thickness; in particular, the strength of thin ice is low and such an icefield moves easily, deforming and dynamically thickening under the action of relatively small external forces, but when the icefield becomes thicker, due to thermal or mechanical growth, the strength of ice increases, leading to large internal stresses and even to total stoppage of the motion. Haapala, Lönnroth, and Stössel (2005) showed that modeling of sea-ice dynamics is highly sensitive to the
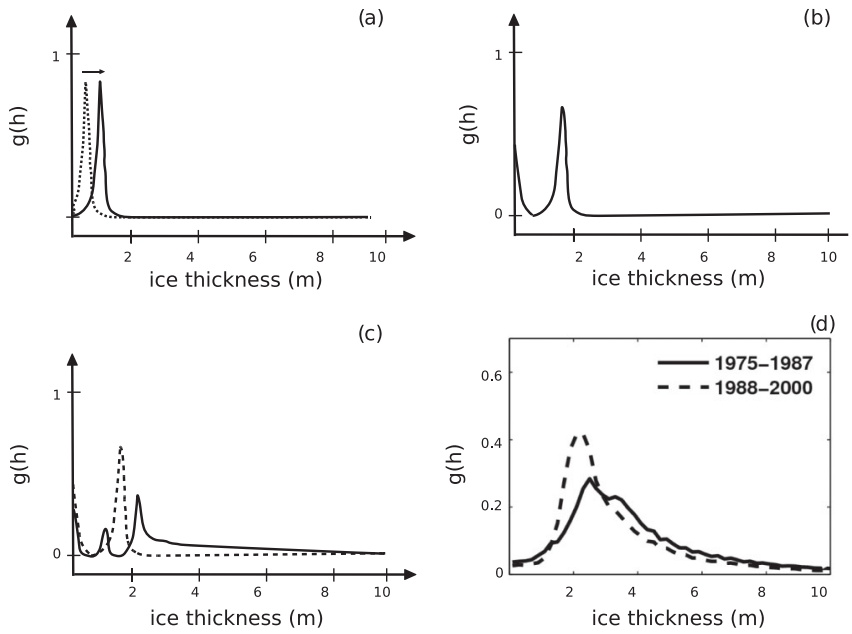

FIG. 20. Conceptual sketches illustrating the life cycle of pack ice by means of the ice-thickness distribution function $g(h)$. (a) The initial freezing of the ocean and the thermodynamical growth of sea ice; (b) $g(h)$ after large-scale fracturing; and (c) the redistribution of pack ice. (d) The observed distribution of sea-ice draft in the Canada basin in spring during two different climatological periods (solid line: 1975-1987, dashed line: 1988-2000). From Oikkonen and Haapala, 2011. 
physical description of the sea-ice thickness distribution, especially of the thin fraction, and found that a multicategory sea-ice thickness model is more responsive to wind forcing than a commonly used two-level approach where ice concentration and mean ice thickness are the prognostic variables.

For process studies, dissipation of the mechanical energy due to the floe flow, floe-lead interaction, and deformation processes such as ridging and rafting (see below) can be resolved explicitly by discrete models (Shen, Hibler III, and Leppäranta, 1986; Hopkins, 1998; Hopkins, Frankenstein, and Thorndike, 2004; Herman, 2011). For large-scale studies, continuum models of rheology need to be used (Feltham, 2008). Until recently, the most feasible approach has been using an isotropic plastic rheology (Hibler III, 1979; Leppäranta, 2004), but satellite observations of large-scale fracturing of pack ice inspired a reformulation of the plastic yield curve (Wang and Wang, 2009), the development of anisotropic rheological models (Wilchinsky and Feltham, 2006), and the formulation of a rheological model based on an elasto-brittle constitution law (Weiss, 2008; Girard et al., 2011).

Thermodynamic growth and decay of ice are a result of heat loss from the ocean to the atmosphere and absorption of the radiative fluxes inside the ice. The physics of thermodynamical ice growth is well captured by the general heat conduction equation. Having in mind that sea ice is a mixture of pure ice, brine, and gas, its thermodynamics follows the theory of a mushy layer (Feltham et al., 2006), which provides a theoretical framework for the interrelation of heat and salt.

An apparent landscape of the ice pack exemplifies the life history of pack ice. Ridges and leads can be identified as generated during a particular event since most of the deformations are irreversible and the life cycle of pack ice can be separated into the following development stages: (1) initial formation of sea ice, (2) fracturing, (3) redistribution, and (4) aging.

\section{Initial formation of sea ice}

The life cycle of pack ice begins with the supercooling of the ocean and the formation of the first crystals of ice, termed frazil ice. Frazil ice can form very thin filaments of ice on the ocean surface or crystals can be mixed in the ocean surface layers by turbulent fluid motion as long as the buoyancy force is not large enough to lift them to the surface (Weeks, 2010). As a result of an accumulation of frazil ice, the first $0.1-1 \mathrm{~cm}-$ thick congealing layer, termed grease ice, is formed on the ocean surface.

In shallow seas under very turbulent conditions, frazil ice can also accumulate on the sea floor, as anchor ice. Frazil-ice formation is not explicitly considered in largescale models, but these processes can be parametrized in the models (Walkington, Morales Maqueda, and Willmott, 2007). The grease-ice layer effectively absorbs wave energy and decreases ocean turbulence, enhancing the accumulation of frazil ice at the surface. In calm conditions, grease ice becomes thicker and more solid due to downward crystal growth at the base of the ice. Otherwise, in addition to thermal growth, ice floes, typically $0.5-2 \mathrm{~m}$ in diameter,

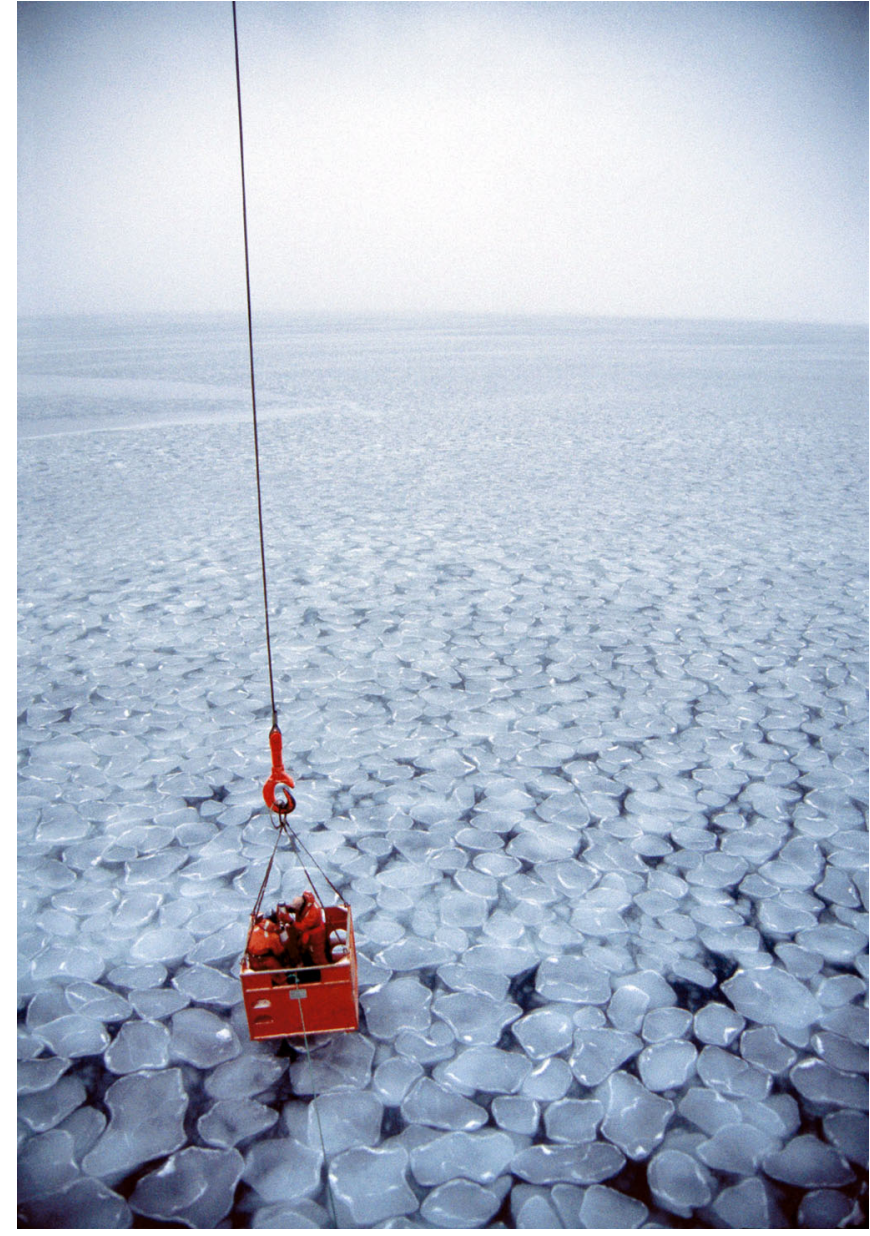

FIG. 21 (color online). Pancake and frazil ice in the Weddell Sea. Pancake thickness here was 5-8 cm and their diameters around $1 \mathrm{~m}$. The image shows the operation of taking samples of frazil ice between the pancake floes.

can be compressed together under wave action to form pancake ice (Doble, Coon, and Wadhams, 2003); see Fig. 21.

At the initial stage, the sea-ice-thickness distribution function $g(h)$ exhibits only one peak. As the ice is thermodynamically growing, the peak shifts ahead in the ice-thickness space; see Fig. 20. Thickening leads to the direction of crystal growth becoming oriented mainly vertically and the size of the crystals becomes much larger. Also the temperature gradient between the top and bottom of the ice increases and the temperature profile changes from linear to nonlinear.

\section{Fracturing}

Fracturing can be regarded as a second stage in the life cycle of pack ice. Traditionally, pack ice has been considered to be a continuum of floes, but pack ice can also be understood to be a continuum of fractures (Hibler III, 2001). A single floe contains thousands of small-scale fractures: thermal cracks, which are caused by the contraction of the sea ice. Recently, Marsan et al. (2011) used a novel seismic monitoring system for recording the deformations of sea ice and found that during the spring, when 


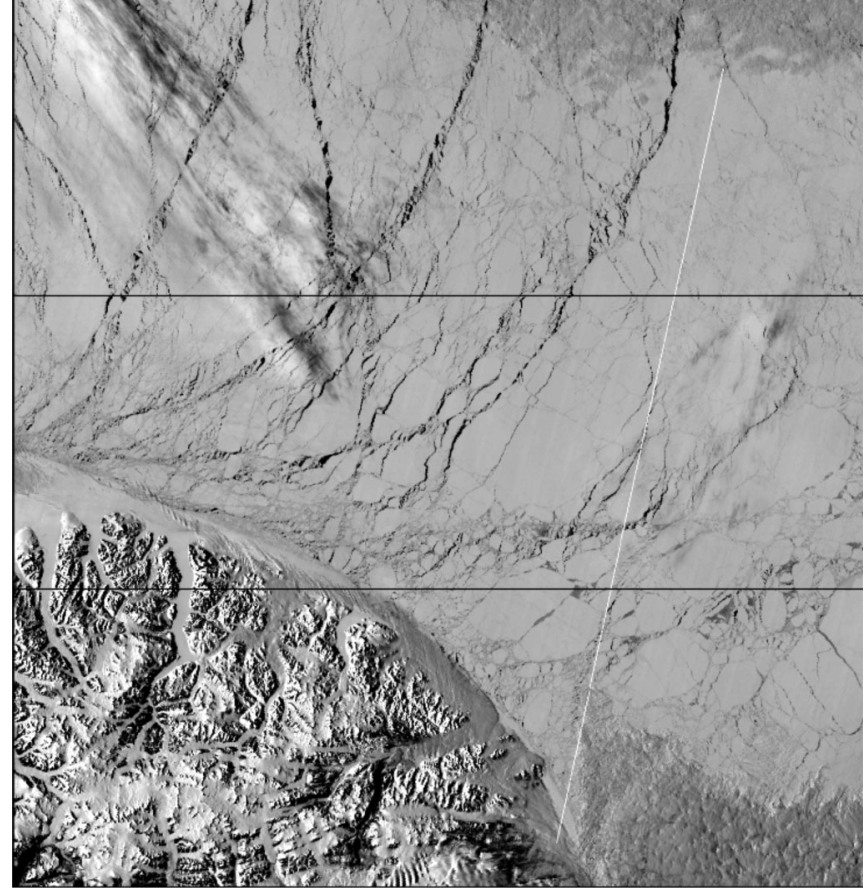

FIG. 22. $385 \times 385 \mathrm{~km}$ satellite (MODIS) image of pack ice in the Lincoln Sea.

large temperature gradients still exist, more than a thousand individual cracking events were observed in a day. Thermal cracking does not change the thickness or concentration of pack ice and hence $g(h)$ remains constant. Large fractures, leads, may extend hundreds of kilometers and are easily detectable from satellite images (see Fig. 22) and could also be detected by the seismic monitoring array (Marsan et al., 2011). These large leads are also called linear kinematic features (LKFs) since as well as the leads being discontinuities in the ice-thickness field, in these locations the seaice velocity field also has strong gradients (Kwok, 2006). The generation of leads changes the open-water fraction of $g(h)$ [see Fig. 20(b)].

In contrast to initial ice formation in open-ocean conditions, freezing of ocean water in leads can occur in very calm and cold atmospheric conditions. In addition to the ice growth at the ice-ocean interface a particular form of ice, frost flowers, can be formed at the thin surface ice cover (Perovich and Richter-Menge, 1994). The surface of thin ice $(\sim 1-2 \mathrm{~cm})$ in a refrozen lead often contains a liquid skin of brine $(\sim 1 \mathrm{~mm})$. Initially pure fresh ice crystals are formed on the ice surface, but during the growth process such frost flowers become covered in brine and are thus extremely saline formations of ice (Perovich and RichterMenge, 1994; Alvarez-Aviles et al., 2008). The presence of a skin of brine has been assumed to be essential for a development of frost flowers (Weeks, 2010), but Style and Worster (2009) showed that frost flowers could be formed by pure ice sublimation into an unsaturated atmosphere. In spite of their marginal fraction of the energy and mass balance of the pack ice, frost flowers are the dominant source of sea-salt aerosols and bromine monoxide, and influence tropospheric chemistry and ozone depletion (Kaleschke et al., 2004).

\section{Redistribution}

The third stage of the pack ice life cycle is the redistribution of the ice pack. In this process some areas of pack ice are compressed together while open water is generated elsewhere in the ice pack. The total mass of the sea ice does not change, but redistribution changes the shape of $g(h)$; see Fig. 20(c). The most common process is that the thinnest ice experiences deformations and produces rafted and ridged ice. Rafted ice is a particular form of deformed ice in which floes partially override each other; ridges are stripelike formations having usually a triangular shape in the vertical dimension. It is known that rafted ice may be rafted many times, and rafted ice could form ridged ice. The deformed ice fraction depends very much on the location; in the Arctic, close to the North Pole the fraction is $20 \%-40 \%$, but in the regions where ice drift is on average convergent the fraction of rafted and ridged ice could exceed $80 \%$.

The deformations begin when external forces cause ice to fail by flexure, buckling, or crushing (Mellor, 1986). Visual observation of the deformed icefield suggests that thin ice predominantly experiences rafting and thicker ice sheets are more often ridged, although thick ice $(h>1 \mathrm{~m})$ is also known to raft. The Parmeter model (Parmeter, 1975) suggests that rafting of an ice sheet is limited by the maximum ice thickness and that a crossover thickness between rafting and ridging (e.g., $17 \mathrm{~cm}$ for the Arctic) relates to the mechanical parameters of the ice.

Laboratory experiments and numerical simulations (Hopkins, 1998; Hopkins, Tuhkuri, and Lensu, 1999; Tuhkuri and Lensu, 2002) have given new insights into the deformations of sea ice. The major finding of ice-tank experiments, where an ice sheet is pushed against another, is that ice sheets of uniform thickness do not form ridges, but a nonuniform ice sheet generates both rafted and ridged ice. Tuhkuri and Lensu (2002) did not find any crossover thickness that would determine whether an ice sheet is rafting or ridging and noted that all ridging events began as rafting events. They distinguished four phases in the development of ridging events in their conceptual model of ridging. First, a force exceeding a threshold value is needed for the initialization of deformation. Then an ice sheet begins to raft. The third phase is keel buildup, and the last phase is lateral growth of the ridge.

Field observations from the Baltic support the conclusion that all ridging events begin as rafting. Babko, Rothrock, and Maykut (2002) also highlighted the importance of rafting in the Arctic and suggested that the crossover thickness should be considerably larger than $17 \mathrm{~cm}$. Recently, Vella and Wettlaufer $(2007,2008)$ derived analytical solutions, based on thin plate theory, for determining the crossover thickness for finger rafting, rafting, and ridging. They found finger rafting (see Fig. 23) to be possible only with ice thicknesses less than $8 \mathrm{~cm}$ and rafting to occur with ice thickness less than $21 \mathrm{~cm}$.

\section{Aging}

The last stage in the life cycle of pack ice is aging. Although the life cycle of an individual ice floe can be considered as a chain of processes, in the ice pack as a whole all these processes are occurring simultaneously. The ice pack 


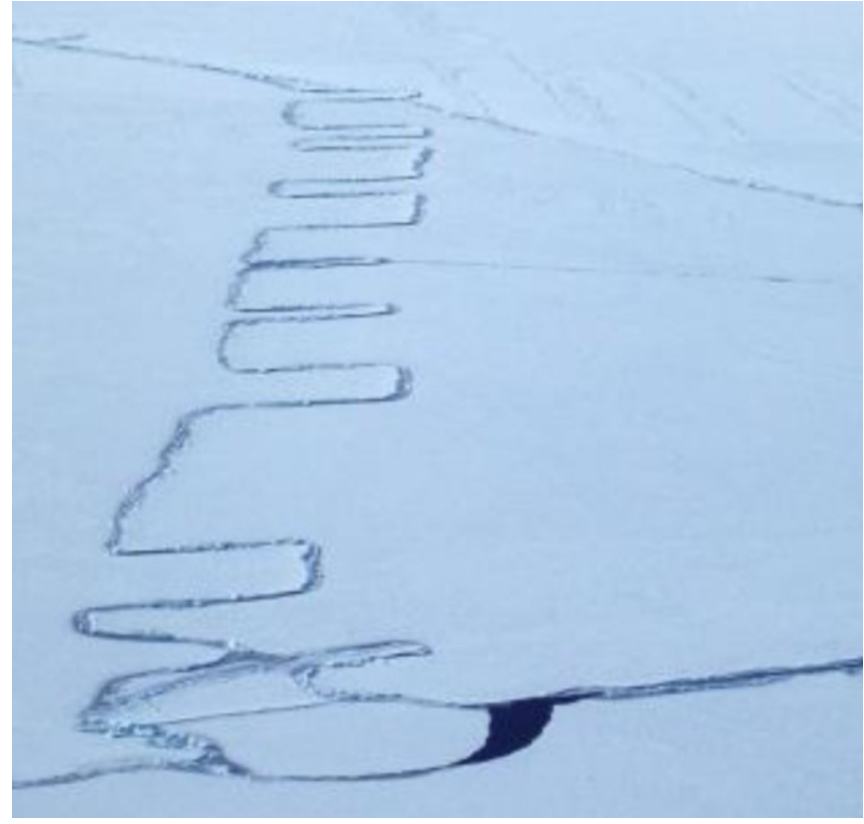

FIG. 23 (color online). Finger rafting in the Lincoln Sea; the fingers are approximately $10-30 \mathrm{~m}$ in length.

is opening and compressing, new ice is formed in the leads, and undeformed ice is thickening. The first-year ridges are weathered at the surface, consolidated internally (Leppäranta et al., 1995; Amundrud et al., 2006), but also melted at their bases due to larger oceanic heat fluxes at the depth of keels (Wadhams, 1992).

In the perennial ice pack, the most prominent process during the summer period is the formation of melt ponds. Melt ponds effectively absorb solar radiation and accelerate the melting of ice since their albedo can be as low as 0.2 (Curry, Schramm, and Ebert, 1995); in comparison, the albedo of snow-cover pack ice is 0.77-0.87. Pedersen et al. (2009) showed that the inclusion of parametrization of the melt ponds to climate models increases the sensitivity of the model, and they suggest that this is the main reason why climate models are not capturing the present observed accelerated melting of the Arctic ice pack.

Persistent drift of ice transports ice to other climatic regions. In the Antarctic, the flow is predominantly toward milder oceanic conditions, resulting in only 1-2 years lifetime for the ice. In the Arctic, the transpolar drift carries pack ice from the North American and Siberian shelf regions to the Greenland Sea via the North Pole, but part of the sea ice is recirculated in the Beaufort Sea gyre. In the 1980s the oldest individual floes were estimated to be 20-30 years old and most of the pack ice was over 10 years old (Rigor and Wallace, 2004). Today, the lifetime of Arctic pack ice is considerably less; most of the ice in the Arctic is first- and second-year ice and older ice exists only in the Lincoln and Beaufort Seas (Rigor and Wallace, 2004; Haas et al., 2008).

The state of the atmospheric circulation, which is the main driving force of sea-ice drift, is commonly described by empirical orthogonal functions (EOFs) of the surface air pressure. The two leading EOFs are the Arctic oscillation (AO) (Thompson and Wallace, 2000) and the dipole anomaly (DA) (Wang et al., 2009). The variability of the AO and DA on a decadal scale affects to a great extent sea-ice circulation and mean sea-ice conditions (Rigor, Wallace, and Colony, 2002; Rothrock and Zhang, 2005; Wang et al., 2009), but also the form of the ice-thickness distribution function (Oikkonen and Haapala, 2011).

The simplifications to sea-ice physics made in different climate models have considerable implications. Many climate models still use rather simplified sea-ice physics, but great progress in the development of the sea-ice component of climate models has been made recently. For example, none of the models selected for the Arctic climate impact assessment (ACIA) resolve sea-ice physics based on the full primitive equations, but now both the community climate system model (Holland et al., 2006) and the Hadley Center climate model (Johns et al., 2006) use the explicitly calculated evolution of several ice categories based on the theory of the sea-ice thickness distribution (Thorndike et al., 1975; Bitz et al., 2001). Holland et al. (2006) concluded that the inclusion of the ice-thickness distribution model in climate simulations enhances the ice albedo feedback and the response of sea-ice thickness to external perturbations.

\section{B. A promoter of the emergence of the first life?}

This review is on ice physics, not biology; it is not our intention to provide a review of physics-biogeochemistry interaction processes and we refer readers interested in that topic to Thomas and Dieckmann (2010). However, the emergence of life from an abiotic environment is an interdisciplinary question of tremendous interest that involves important physical and chemical considerations in the selforganization necessary for life to arise; it is the physics and chemistry of biogenesis in ice that we concentrate on here.

Many different environments and processes facilitating the first steps to life have been proposed (Orgel, 1998; Schopf, 2002; Davies, 2003; Bernstein, 2006; Schulze-Makuch and Irwin, 2008). As Orgel (1998) commented, "the problem of the origin of life on the Earth has much in common with a well-constructed detective story. There is no shortage of clues pointing to the way in which the crime, the contamination of the pristine environment on the early Earth, was committed. On the contrary, there are far too many clues and far too many suspects. It would be hard to find two investigators who agree on even the broad outline of events." Among these many suspects, we can distinguish those that involve a hot origin of life on Earth from another group favoring a cold origin. One hypothesis that has been put forward within the context of a cold origin of life, one that interests us in the present setting, is that life may have originated in ice.

Price (2007) provided a very broad base of references defending the possibility of ice as promotor for life. While, as mentioned in Sec. III.C, some researchers lean toward the extraterrestrial origins of prebiotic material (Bernstein, 2006), for which astrophysical ices, including but not limited to water ice, are important, others consider the possible formation of prebiotic material in sea ice (Trinks, Schröder, and Bierbricher, 2005). Here we discuss the arguments related to sea ice on the early Earth while remembering that these arguments pertain too to other similar astrophysical environments. 
Owing to its being a multiphase system, sea ice reacts under small temperature variations with strong property changes. In this way sea ice renders many conditions and processes conducive to the generation of life, e.g., low temperature, the enrichment of compounds, the presence of small membranous compartments, chromatographic compound separation, temporal and spatial variations of $p \mathrm{H}$, electrical potentials and ionic strength values together with catalytic effects on the surfaces of ice crystals, and the presence of numerous small mineral particles always embedded in sea ice. The above described properties of sea ice imply that it forms a complex system that may have some importance for some of the first steps toward the emergence of life.

Extant cells are autonomous: All properties and structures are an integral part of the cellular reproduction apparatus. But most researchers agree that the emergence of life was a process with many intermediate stages of increasing sophistication and complexity to reach this autonomous state. In the early steps to life, many features, such as the building of compartments and catalysis, presumably had to be provided by the environment, and in order to ensure the necessary continuity of the self-organization process that eventually led to life, had to be present in a quotidian manner. Investigations into the stability of RNA structures in cold environments (Moulton et al., 2000) and experiments performed at low temperatures under eutectic conditions (Stribling and Miller, 1991; Liu and Orgel, 1997; Monnard, Kanavarioti, and Deamer, 2003; Vlassov et al., 2004) indicate that the seaice matrix might be an environment well suited to support processes that could finally lead to living systems.

Ice-reactor experiments have been carried out on artificially frozen sea water over time intervals of up to $1 \mathrm{yr}$ (Trinks, Schröder, and Bierbricher, 2005). Frozen sea water is a multiphase system which contains small ice clusters of frozen, salt-free water between which liquid, highly salty brine runs. Almost all ionic compounds have extremely low solubility in ice; as ice grows, ice polycrystals reject common ions into planar boundaries and, if their concentration is high enough, further concentrate them from two-grain boundaries into three-grain boundaries or veins (see Sec. II.D). Small gas bubbles, and additionally, various mineral particles and salt crystals, are embedded in a large number, approximately $10^{15} \mathrm{~m}^{-3}$, of compartments (Trinks, Schröder, and Bierbricher, 2005). The boundary layers between the solid clusters of ice and brine consist of flexible, less than $1 \mu \mathrm{m}$ thick, membranes (Engemann et al., 2004), which exist above temperatures of $200 \mathrm{~K}$ (Wei, Miranda, and Shen, 2001) and are stable for several minutes even after the ice has completely melted. Potential differences of up to $50 \mathrm{mV}$ and local gradients of $p \mathrm{H}$ from 6 to 8 have been observed (Mazzega et al., 1976; Bronshteyn and Chernov, 1991). The strong ultraviolet light that irradiated the young Earth (Zahnle and Walker, 1982) will have influenced the upper layer of sea ice to a depth of about $10 \mathrm{~cm}$. Under typical local temperature gradients of up to $10 \mathrm{~K} / \mathrm{hr}$ and $10 \mathrm{~K} / \mathrm{m}$, pressure differences provoke a steady movement of the brine, which leads to chromatographic effects due to the interaction of dissolved compounds with the ice surfaces. Small temperature variations have drastic effects on the constitution of the multiphase system of sea ice. They lead to sudden melting and freezing, degassing and dissolving of gas, crystallization, and redissolving.

The concentration of prebiotic compounds in the oceans of the young Earth is estimated to have been less than a few $\mathrm{mg} / \mathrm{l}$ (Stribling and Miller, 1986) and effective enrichment processes must have been indispensable steps toward selforganization. As discussed above, when sea water freezes, dissolved compounds are enriched due to the growth of freshwater ice, while salts and other ingredients concentrate in the liquid portion that fills the small cavioles. The concentration of this brine is enriched by a factor of 5 to 10 , compared to unfrozen sea water, when temperatures of -15 to $-20{ }^{\circ} \mathrm{C}$ are reached. At temperatures below $-25^{\circ} \mathrm{C}$, and depending on their concentration related to the inorganic load, precipitation of organic ingredients (e.g., amino acids or RNA) starts. The dissolved ingredients have been observed to form aggregates in brine. Biomacromolecules such as RNA spontaneously hydrolyze rather than polymerize in dilute aqueous solutions; however, because of their low water activity, veins in polycrystalline ice promote polymerization rather than hydrolysis. Because of the cyclical changes of temperature in natural sea ice these precipitates partially redissolve and precipitate once more, favoring the growth of more crystalline structures. In this way dissolved ingredients in the sea water, though low in concentration $(<1 \mathrm{mg} / \mathrm{l})$ under the freezing ice sheet, might form solid aggregates with typical local densities approaching approximately $1 \mathrm{~kg}$ per liter, constituting an enrichment of compounds by a factor of $10^{6}$ (Trinks, Schröder, and Bierbricher, 2005).

Separating the melange of prebiotic compounds is essential for the synthesis of larger molecular structures, because some molecules inhibit the synthesis of others (Shapiro, 2000). Sea ice is an environment with some separation power; it has the properties of a chromatograph in which the ice surface acts as the stationary phase and the liquid brine as the mobile phase (Dasgupta and Mo, 1997). The experiments that have been conducted show that this is not enough to separate totally, for example, amino acids from carboxylic acids or amines, important for some prebiotic synthetic steps. Nevertheless, separation processes in sea ice, in addition to supposed similar other features in the environment of the young Earth, may have been instrumental in priming the generation of life.

The reaction of prebiotic molecules will have preferably taken place in an environment which supported synthetic processes. Condensation is a typical reaction in biological systems and should have played an important part in the prebiotic chemistry to build polymers from amino acids, RNA precursors, or others. The growth of pure ice crystals in sea ice during freezing can be regarded as a water consuming process possibly favoring such condensation reactions. In addition, a high concentration of $\mathrm{NaCl}$ has been found to initiate the condensation of amino acids into peptides, known as the salt-induced peptide formation reaction (Schwendinger et al., 1995; Rode, 1999). The surfaces of ice, as well as of mineral particles, always present in sea ice, are known for their catalytic properties (Ferris, 1998; Graham and Roberts, 2000). Thus catalysis may also be considered to be involved in reactions in sea ice. 
Experiments in sea ice have been performed with solutions of alanine, phenylalanine, serine, tryptophan, and isoleucine. After a few days of freezing under ultraviolet light, a material supposed to be peptides of high molecular weight was generated and formed a reticular structure that filled the cavioles and was partly attached to their walls. After complete melting of the ice the amino-acid condensate was found to remain as a reticular structure resistant to dissolution and even to hydrolysis in hydrochloric acid (Trinks, Schröder, and Bierbricher, 2005).

Biologically active molecules that are synthesized need to remain together in structured cellular compartments to be able to act in further evolutionary steps, e.g., to take part in self-organization during cyclic chemical processes, as proposed by Eigen and Schuster (1979). A great variety of cellular envelopes is contained in the sea-ice matrix. The channels at low temperatures $\left(<-20^{\circ} \mathrm{C}\right)$ reach typical dimensions of about $10-50 \mu \mathrm{m}$ in diameter. The step from the wall-coated ice cells to the generation of autonomous vesicles composed of polymers from prebiotic molecules appears to take place surprisingly easily when ice melts (Trinks, Schröder, and Bierbricher, 2005). During freezing, membranelike coverings are constructed that follow the contours of the small ice channels [see Figs. 24(a) and 24(b)]. During melting these layers readily form clusters or vesicles [see Fig. 24(c)] that float into the aqueous medium without being redissolved [see Fig. 24(d)].

Thus the freezing processes of sea water support chemical reactions, enrich and sort prebiotic compounds, and provide a medium for vesicle formation. Sea ice may be a matrix that is able to promote essential steps on the way to life; see Fig. 25 for a sketch. Sea-ice vesicles, filled with the products of icematrix-supported prebiotic chemical processes, and released
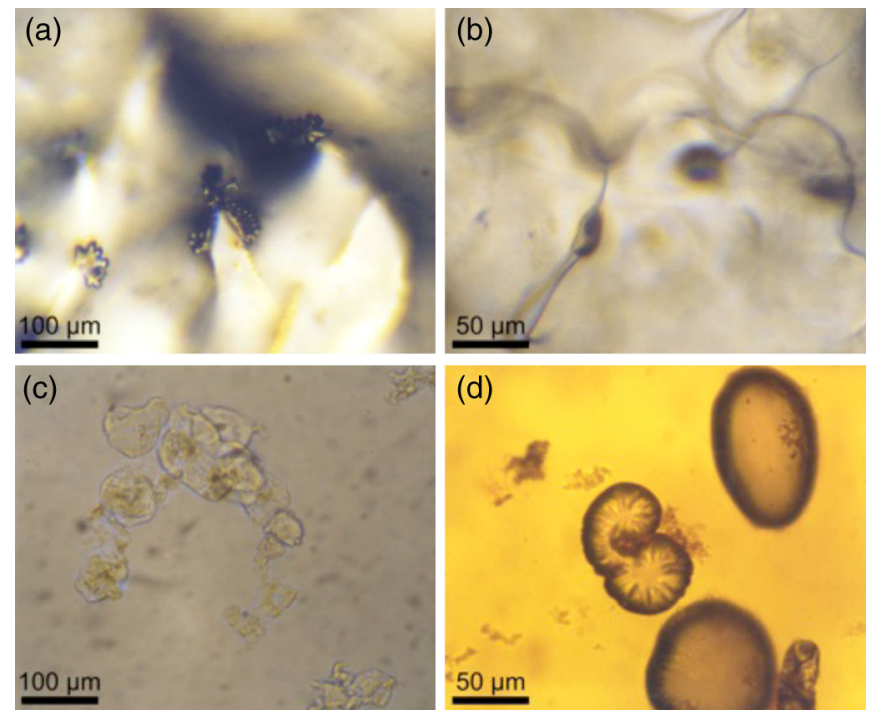

FIG. 24 (color online). Membranes and vesicles in ice: (a) Phosphatidylcholines distributed in sea water form membranes on ice surfaces and at the edges of mineral particles. (b) Formation of vesicles in sea ice by phosphatidylcholines during partial melting. (c) Peptide clusters of phenylalanine form vesicles in the ice. (d) Yeast RNA dissolved in sea water and frozen in sea ice forms vesicles under the influence of ultraviolet light.

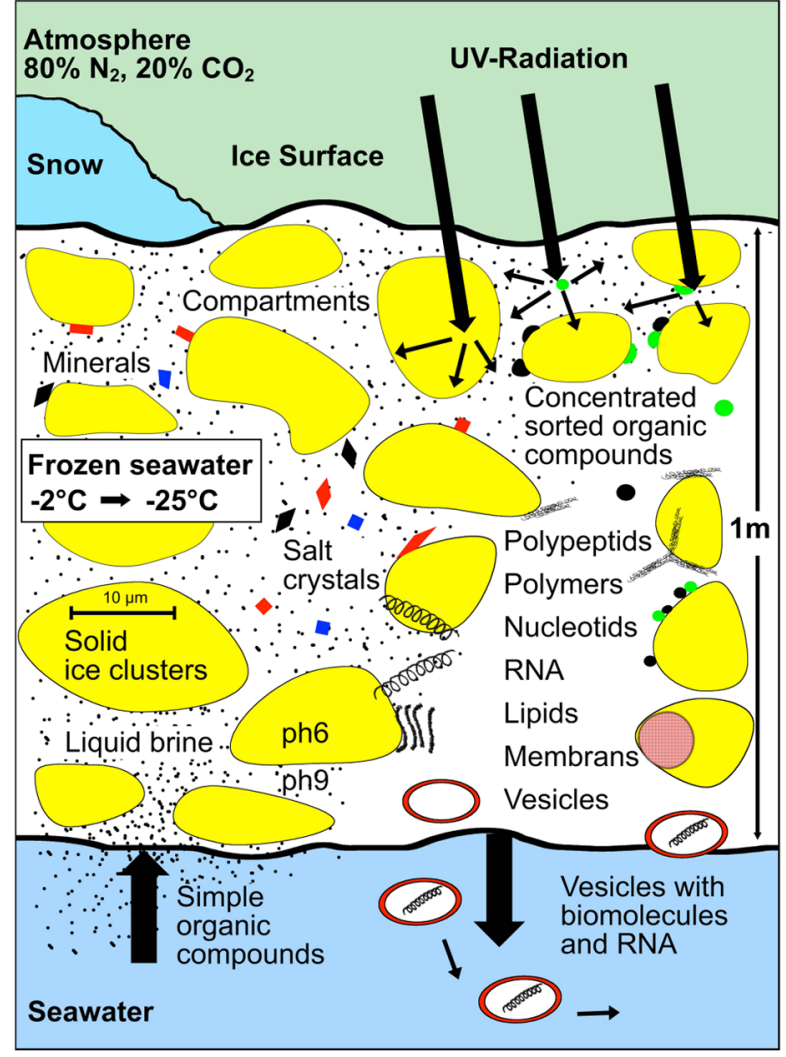

FIG. 25 (color online). Proposed life-promoting reactions in sea ice: Simple prebiotic organic compounds were transported from sea water into the body of the ice (typical thickness 1-2 m); in the icy medium the reagents were sorted, separated, and concentrated; chemical reactions led to polypeptides, RNA structures, and membranes; the membranes formed vesicles embedding biomolecules such as RNA and others; and finally the vesicles left the ice and floated into the sea water beneath. From Trinks, Schröder, and Bierbricher, 2005.

into the prebiotic aqueous environment underneath the ice sheet, may have floated in sea water to other locations on Earth until finally a cell fit for life had evolved.

As with several other themes we discuss in this paper, this issue could have appeared in another place, in the astrophysical ice section, Sec. III; as mentioned there, similar ideas are being discussed in the astrophysical ice community with regard to the possible emergence of life on icy bodies in the Solar System and beyond. The presence of water is widely held to be crucial for the generation of life anywhere in the Universe. While the role of ice in the emergence of life on Earth is not yet clear, there are many other places in the Universe in which it may be of relevance. Planets and moons are, in many cases, thought to possess ice in their polar regions (e.g., Mars), while others seem to be covered by frozen oceans (e.g., Europa), and cometary ice may contain water from radioactive heating. Just as life is frequently preserved in the water beneath the icy crust of a frozen pond in winter, so perhaps there may be life in the ice-coated oceans of other worlds that can exist because of this quirk of ice physics that ice Ih floats on water. It may then be that ideas on ice as a cradle for life may be of interest in extraterrestrial contexts [see, e.g., Price (2010)]. 


\section{PERSPECTIVES}

If you watch a glacier from a distance, and see the big rocks falling into the sea, and the way the ice moves, and so forth, it is not really essential to remember that it is made out of little hexagonal ice crystals. Yet if understood well-enough the motion of the glacier is in fact a consequence of the character of the hexagonal ice crystals. But it takes quite a while to understand all the behavior of the glacier (in fact nobody knows enough about ice yet, no matter how much they've studied the crystal). However the hope is that if we do understand the ice-crystal we shall ultimately understand the glacier.

\section{Richard Feynman, The Character of Physical Law}

So what are the main open questions in research on ice today?

If we begin by examining the issue of molecular structures, we find that there are even uncertainties remaining concerning some aspects of the ice phase diagram, especially in relation to understanding the mechanism of hydrogen ordering by acid or base doping and obtaining definitive information on the various ground-state structures. The possible existence of small ordered nuclei within the disordered phases is an interesting speculation. So-called cubic ice remains a particularly interesting problem, one which perhaps has some relation to the polytypes observed in systems such as silicon carbide. And understanding the details of both the molecular-level and intermediate-range structures of the amorphous ices is a particularly problematical issue that is far from being resolved.

In the field of astrophysical ice, the forthcoming years will be marked by the collection of new and exciting data from several space missions sent to explore Solar System bodies. Among them, it is worth mentioning the New Horizons mission (NASA) presently heading toward Pluto through the Kuiper belt and Rosetta (ESA), a spacecraft that will accompany comet Churyumov-Gerasimenko for more than a year and that will deploy a lander, loaded with nine instruments, on the surface of the comet. As we go to press, the Messenger mission to Mercury and the Dawn mission to 4 Vesta and Ceres will likely answer the questions of the presence of ice on these bodies. Data collected on such space missions will be added to the large amount of information already gathered by the very successful instruments used to explore space, such as the space telescope Hubble and the Cassini-Huygens mission, to name but two. This wealth of knowledge should enable the astrophysical community to address some of the most intriguing questions related to astrophysical ice as yet unanswered. For instance, what is the structure of cometary nuclei: Do they contain crystalline as well as amorphous water ice, and are there other volatile species trapped in the ice? Will the presence of clathrates be definitely confirmed, and if so, in which type of ice systems and astrophysical bodies? The unravelling of these mysteries renders the near future a very exciting time for research in astrophysical ices.

In the longer term, there is also the interesting question of the presence of the various ice phases in astrophysical environments. At present, almost everything remains speculation, as there is no significant difference between the infrared spectra of ice Ih and ice Ic, and high-pressure ices inside planets or moons are not accessible to remote-sensing IR spectroscopy. The conditions for the formation of any of the high-pressure phases of ice are unlikely to occur naturally anywhere on Earth; or perhaps that is too hasty a judgment? Bina and Navrotsky (2000) suggested that ice VII might be present in subduction zones of the Earth's crust. Elsewhere in the Solar System, many conditions could well exist under which these high-pressure phases could have formed. For example, it has been argued (Sohl et al., 2002) that the interior of Jupiter's moon Ganymede could consist of successive layers of ices Ih, III, V, and VI. Similarly, the other largest icy Solar System moons, Callisto and Titan, might have ice VI in their depths, and layers of other ice phases in onion fashion toward the surface. The centers of somewhat smaller bodies, such as the trans-Neptunian objects Pluto and Eris, might too consist of a mixture of rock and high-pressure ices. These high-pressure phases are all disordered phases. What about the ordered ones, whose presence could be astrophysically significant bearing in mind the general differences in physical (e.g., mechanical) properties of ordered and disordered phases? At present, it seems unlikely that the ordered phases will be found in the Solar System, given the ranges of temperature and pressure expected in icy Solar System bodies. So perhaps we shall have to wait for developments in exoplanets, but surely, somewhere in space, in some body orbiting some distant star or planet, the pressure and temperature conditions are right for ice $\mathrm{XV}$ ?

An interesting related question to that of astrophysical ice phases, around which work is ongoing (Durham and Stern, 2001; Greve, 2006), is about the rheology or glaciology of ices in a planetary context; e.g., are there glaciers on Pluto? The deformation and flow of ice at low temperature is most likely qualitatively different to that on Earth, so that Glen's flow law cannot be extrapolated in a simple manner to low temperatures. As well as ice Ih, one has to consider flow in amorphous ices as well as in ice Ic and the high-pressure phases, and grain size may well affect flow to a considerable extent. It will be useful to have experimental results from the laboratory to compare with models, but at present there are no low-temperature data available, to say nothing of data at low temperature and high pressure, and they will be very difficult and time consuming to obtain. Astrophysical ice rheology is important to tell us the minimum size at which an icy body becomes round under its own gravity; the figure is likely different than for a rocky body, and this question of roundness impinges on the current definition of a dwarf planet.

Further planetary geophysical issues include cryovolcanism and cryotectonics. Cryovolcanoes, or ice volcanoes, are structures that are proposed to function analogously to terrestrial volcanoes, but their eruptions are of molten ice, water, rather than lava, molten rock. These have been sought on various bodies in the Solar System; at present, it is thought that features seen on Titan are good candidates, but unam- 
biguous proof for cryovolcanism anywhere in the Solar System is still lacking. Cryotectonics, plate tectonics with ice, is clearly a possibility for bodies that have an icy crust over a liquid water layer undergoing convection. Might mixtures of ices (water ice and other frozen volatiles) permit there to be oceanic and continental plates as with terrestrial plate tectonics? That is as yet speculation, but cryotectonic activity has been observed on Enceladus, at ice fractures near its south pole. Europa too may have tectonic activity on what is believed to be an ice crust over an ocean of water; moreover, among many speculations, one is that some features on the Europan surface may be due to the ice crust itself convecting in the sort of slow flow we discussed above. Last, there is the puzzle of the ice ridge on Iapetus; this mountainous ridge, up to $20 \mathrm{~km}$ high, circles the equator on the Saturnian moon of diameter $1400 \mathrm{~km}$, which consequently looks like a walnut. No theory for its formation seems completely satisfactory; it remains a mystery.

A great many open questions remain to be answered in the fascinating field of research on ices in the Universe and their relationship with the evolution of matter and eventually life on Earth (and beyond?). Among them we highlight the following: Is the structure, and thence the spectral properties, of the interstellar water ice-rich mantles well reproduced in laboratory experiments? In the latter water ice is usually accreted from the gas phase onto cold substrates. In space oxygen and hydrogen atoms recombine by surface chemical reaction on the grain surfaces. Laboratory experiments focused on the study of the spectral characteristic of ices obtained by surface reactions are urgently required. Has the water ice that dominates the crust of the bodies in the outer Solar System a terrestrial analog? What we really observe is the outer skin (from micrometers to millimeters depending on the wavelength) of a given object. The structure and composition of those layers are continuously altered by ion irradiation and micrometeorite bombardment. Can we reproduce something similar on Earth? Comets are an aggregate of ices and dust. At which ice-dust ratio do the physical properties of ices change significantly? How does one measure parameters such as thermal conductivity, compressibility, etc., for such a macroscopic (kilometer-scale) aggregate?

The molecular structure of ice sets the stage from which physical properties follow such as the anisotropy of ice Ih with respect to deformation. What is the most stable structure? This is not so clear in ice; many competing solutions exist for minimizing free energy. This comes into play when nucleating the frequently occurring metastable structures. Ice nucleation is far from being understood; nor is the growth of ice crystals, in particular, under atmospheric conditions. One of the main open questions in understanding Earth's radiation balance, and thus climate change, is related to ice nucleation. Atmospheric ice nuclei can trigger cloud formation and precipitation, having an impact on both the global radiation balance and the water cycle. The impact on the balance is still debated, as, on the one hand, ice clouds have a higher albedo than liquid water, so they should cool the climate (Mishchenko et al., 1996), but, on the other hand, they are more likely to precipitate and so reduce total cloud albedo (Lohmann, 2002). The light scattering and reflection of ice particles in the atmosphere is not yet well described, since these optical effects depend on the particle morphology (size and shape), which often varies with the atmospheric conditions and is difficult to model.

In general, the entire microphysics of ice clouds is still under consideration. In particular, heterogeneous ice nucleation is not well parametrized. For many nuclei, even the nucleation rates are not available. This is especially true for biological aerosols, which only recently have moved into the center of interest of the atmospheric ice community. Depending on the nucleation mechanism, morphology and phase composition might also change. The latter is also a problem for field measurements, since up to now there are no instruments available that can determine the crystallographic nature of an ice or hydrate phase beyond the well-known, but sometimes equivocal, spectroscopic specifications. Similar difficulties exist for obtaining nanometric images of atmospheric snow crystals; such microstructural information is needed, for example, to confirm and quantify the suggested role of complex snow-crystal morphology on vapor pressure and chemical reactivity [see, e.g., Gao et al. (2004)]. We have good evidence of ice surfaces being important for various chemical reactions, but there remain many common atmospheric substances, from the biogenic to anthropogenic, whose chemical behavior remains to be fully quantified, yet this leads us outside the field of our review.

Snow forms a wealth of different growth morphologies for which our understanding is mostly only qualitative. Morphologies are important regarding the sedimentation speed (Wang, 2002) of snow crystals and their interaction with atmospheric constituents during formation and fall, but also on the ground. The transfer functions from atmosphere to snow are often known only approximately while they are of primary importance for decoding the information contained in deep ice cores to reconstruct climate history. Related to this is the question of where exactly photoreactions in environmental ice take place and how these can best be parametrized in models. Morphologies are also important for our understanding of processes in planetary science and astrophysics; again photochemistry on ice surfaces is one of the open fields in this context.

In terrestrial ice open questions range from the macroscopic behavior of large ice sheets down to the microscopic uptake of atmospheric trace gases: Generally, the flow of glaciers and its relation to microscopic ice properties is qualitatively well understood (Schulson and Duval, 2009). However, important details on how the microscopic properties of single ice crystals link to the creep behavior of polycrystalline ice are still unclear. Self-consistent models developed in the field of materials science have now been applied to ice [see, e.g., Lebensohn et al. (2009)], yet dynamic recrystallization, important for larger glaciers and ice sheets, still awaits its full implementation. Theoretical advances in modeling are helped by new techniques such as cryo-electron backscatter diffraction (EBSD) (Obbard, Baker, and Sieg, 2006), which are starting to provide access to microstructural details of recrystallization processes. Linking microstructural information to geophysical signals such as radar imaging and seismic data is another formidable challenge in ice-core research; if successful it would enable remote mapping of microstructural effects in large ice sheets 
(Drews et al., 2009). Also open to debate are the reasons that explain the observed accelerated glacier flow along polar coasts and the role of sea ice. In this respect it was proposed that thermal convection may play a role in the origin of ice streams (Hughes, 2009). Also related to glaciers are open questions regarding the sintering process of glacial snow. A key question is to understand quantitatively the time offset of ice and neighboring closed-off air bubbles in deep ice cores. A deeper understanding of the dynamics during the transformation of glacial snow to ice, where air bubbles are finally trapped, would help to understand better the timing at which various possible climate forcings acted in the past. Smallscale dynamics are also of interest in understanding in detail the mechanism of icicle formation (Neufeld, Goldstein, and Worster, 2010; Chen and Morris, 2011).

With regard to sea ice, the most important research questions are as follows: What mechanisms control the variability and change of sea ice? What is the relationship between large- and small-scale processes? What governs the processes of interaction between snow, sea ice, and atmosphere? What is the nature of the interaction between the sea ice and radiation? How do biogeochemical processes depend on the physical properties of sea ice? Keeping in mind the welldocumented changes now occurring in the ice pack of the Arctic Ocean, open questions remain on the differentiation of anthropogenic forced climate signals from natural variability, the separation of the relative importance of atmospheric and oceanic forced change, and on understanding what mechanisms control the variability and change of sea ice. Our lack of a comprehensive understanding is partly due to limited observational capabilities, and new satellite missions such as CryoSat-2 and enhanced ocean monitoring activities will provide much more accurate and more numerous data to explore the physics of the Arctic climate system. Small-scale processes are known to be important also at the climate scale, but these processes are usually neglected or poorly parametrized in the large-scale models, and the phenomena they generate have received hardly any attention. To give just one example, consider sub-ice convection, during which ice may form around the draining cold brine plumes under floating ice, producing tapered hollow structures up to meters in length termed sea-ice stalactites (Paige, 1970). A fascinating open question of sea-ice physics is to establish a theoretical framework between the properties of ice, local-scale processes (e.g., fracturing, floe-floe friction, dissipation of kinetic energy during deformation), and largescale ice motion. This kind of research could enable a statistical-dynamical parametrization of the subgrid scale processes for climate models.

We should mention here the methane hydrates, sometimes referred to as "burning ice" due to a number of similarities with ice Ih, that exist in sediments of the ocean floor. In these crystalline compounds the water molecules form a threedimensional structure consisting of cages containing mainly methane molecules, justifying the term "clathrates." The amount of methane stored in hydrates is considered to be at least comparable to that in existing natural gas deposits, but it is unclear at present whether and how much of these gas hydrates are economically recoverable. These clathrates are stable on Earth only under the high pressures and at the fairly low temperatures of a few degrees Celsius prevailing in sediments on the sea floors of continental margins. The study and control of the stability of methane hydrates is thus likely of importance for their use as an energy resource. Because of their potential nuisance-upon decomposition methane gas may escape in large amounts into the atmosphere-methane hydrates are also a concern in environmental studies and climate change predictions. Gas hydrates form a very large and independent field of research (Sloan and Koh, 2008); in the marine context on Earth they belong to the cryosphere only marginally, via air clathrates in ice sheets and the likely occurrence of methane hydrates within permafrost. However, in the planetological context the distinction between ice and clathrate hydrates is less strict. Conclusive evidence for the existence of gas hydrates on celestial bodies is still missing, since with infrared spectroscopy they are very hard to distinguish from a mixture of water ice and gases such as $\mathrm{CO}_{2}$. Moreover, the formation kinetics are very slow at low temperatures. A fair amount of discussion on the role of $\mathrm{CO}_{2}$ clathrate hydrates in the planetological context can be found in Kargel (2004), but see also a more critical appreciation by Falenty et al. (2011).

In our view across the icefields we have seen that there are many open questions in different aspects of our knowledge of the behavior of this small molecule, water, in its solid state as ice. What is more, many of these frontier questions on ice structure, patterns, and processes are interrelated; multidisciplinary and interdisciplinary research is needed to resolve them. In this regard, an approach we commend, which may be applied fruitfully to ice [see, e.g., Faria et al. (2009)], is the study of the interaction of internal structure, form, and the environment. Feynman (1967), whose words we set at the start of this section, was extremely prescient in highlighting this multiscalar approach from the atomic to the geological scale.

We conclude by reproducing the first verse of a poem Maxwell (1871) composed for the meeting of the British Association for the Advancement of Science in 1871 (Booth, 1999). In just a few lines he mentions three physical properties of this complex substance-pressure melting or regelation, its tendency to form dendritic crystals, and veins and nodes within a polycrystal - that are still topics of current research. Today, as in the 19 th century, ice physics continues to fascinate.

I come from fields of fractured ice, Whose wounds are cured by squeezing, Melting they cool, but in a trice, Get warm again by freezing.

Here, in the frosty air, the sprays

With fernlike hoar-frost bristle,

There, liquid stars their watery rays

shoot through the solid crystal.

James Clerk Maxwell, To The Chief Musician Upon Nabla: A Tyndallic Ode

\section{ACKNOWLEDGMENTS}

We thank Christoph Salzmann for Figs. 4 and 5, Larry Wilen for Fig. 7, Heidy Mader for Fig. 8(a), Martin Doble for 
Figs. 21 and 22, Mike Steele for Fig. 23, and Jean-Loup Bertaux, Bruno Bézard, Buford Price, and David Thomas for useful discussions. This article is fruit of the European Science Foundation workshop Euroice 2008 held in Granada, Spain from 1-4 October 2008 attended by most of the present authors; we thank the ESF for its financial support. That workshop was itself born from an earlier Spanish national project, Hielocris, financed by the Consejo Superior de Investigaciones Científicas; we thank the CSIC for its financial support. In addition we acknowledge funding from FWF, Austria (No. P23027); MINCINN, Spain (No. FIS2010-16455, No. PR2010-0012, and No. FIS201022322-528C02-02); and SNSF, Switzerland (No. 200021 121857). Neutron-scattering studies played a major role in molecular-level ice physics and we thank ILL (France) and ISIS (UK) for the neutron beam time that has made much of that work possible.

\section{REFERENCES}

Abbatt, J. P. D., 2003, "Interactions of atmospheric trace gases with ice surfaces: Adsorption and reaction," Chem. Rev. 103, 4783.

Abbatt, J. P. D., T. Bartels-Rausch, M. Ullerstam, and T. J. Ye, 2008, "Uptake of acetone, ethanol and benzene to snow and ice: effects of surface area and temperature," Environ. Res. Lett. 3, 045008.

Al-Halabi, A., A. W. Kleyn, and G. J. Kroes, 1999, "New predictions on the sticking of $\mathrm{HCl}$ to ice at hyperthermal energies," Chem. Phys. Lett. 307, 505.

Al-Halabi, A., A. W. Kleyn, and G. J. Kroes, 2001, "Sticking of $\mathrm{HCl}$ to ice at hyperthermal energies: Dependence on incidence energy, incidence angle, and surface temperature," J. Chem. Phys. 115, 482.

Allamandola, L. J., S. A. Sandford, A. G. G. M. Tielens, and T. M. Herbst, 1992, "Infrared spectroscopy of dense clouds in the $\mathrm{C}-\mathrm{H}$ stretch region-methanol and 'diamonds'," Astrophys. J. 399, 134.

Alley, R. B., 1992, "Flow-law hypotheses for ice-sheet modeling," J. Glaciol. 38, 245.

Alpert, P. A., J. Y. Aller, and D. A. Knopf, 2011, "Initiation of the ice phase by marine biogenic surfaces in supersaturated gas and supercooled aqueous phases," Phys. Chem. Chem. Phys. 13, 19882.

Alvarez-Aviles, L., W. R. Simpson, T. A. Douglas, M. Sturm, D. Perovich, and F. Domine, 2008, "Frost flower chemical composition during growth and its implications for aerosol production and bromine activation," J. Geophys. Res. 113, D21304.

Amundrud, T. L., H. Melling, R. G. Ingram, and S. E. Allen, 2006, "The effect of structural porosity on the ablation of sea ice ridges," J. Geophys. Res. 111, C06004.

Anastasio, C., and T. Robles, 2007, "Light absorption by soluble chemical species in Arctic and Antarctic snow," J. Geophys. Res. 112, D24304.

Andersson, P. U., M. T. Suter, N. Marković, and J. B. C. Pettersson, 2007, "Water condensation on graphite studied by elastic helium scattering and molecular dynamics simulations," J. Phys. Chem. C 111, 15258.

Andrews, J. T., 2007, "Glaciers, Oceans, Atmosphere and Climate," in Glacier Science and Environmental Change, edited by P. G. Knight (Blackwell Publishing, Malden, MA), 1st ed., p. 95.

Arakawa, M., H. Kagi, and H. Fukazawa, 2010, “Annealing effects on hydrogen ordering in KOD-doped ice observed using neutron diffraction," J. Mol. Struct. 972, 111.
Ariya, P. A., et al., 2011, "Snow-a photobiochemical exchange platform for volatile and semi-volatile organic compounds with the atmosphere," Environmental Chemistry 8, 62.

Arstila, H., K. Laasonen, and A. Laaksonen, 1998, "Ab initio study of the gasphase sulphuric acid hydrates containing 1 to 3 water molecules," J. Chem. Phys. 108, 1031.

Avron, J.E., and D. Levine, 1992, "Geometry and foams-2D dynamics and 3D statics," Phys. Rev. Lett. 69, 208.

Ayotte, P., R. S. Smith, K. P. Stevenson, Z. Dohnálek, G. A. Kimmel, and B.D. Kay, 2001, "Effect of porosity on the adsorption, desorption, trapping, and release of volatile gases by amorphous solid water," J. Geophys. Res. [Solid Earth Planets] 106, 33387.

Babko, O., D. A. Rothrock, and G. A. Maykut, 2002, "Role of rafting in the mechanical redistribution of sea ice," J. Geophys. Res. 107, 3113.

Bailey, M., and J. Hallett, 2002, "Nucleation effects on the habit of vapour grown ice crystals from -18 to -42 degrees C," Q. J. R. Meteorol. Soc. 128, 1461.

Bailey, M., and J. Hallett, 2004, "Growth rates and habits of ice crystals between -20 degrees and -70 degrees C,' J. Atmos. Sci. 61, 514.

Balci, F. M., and N. Uras-Aytemiz, 2011, "Auto-ionization of $\mathrm{HNO}_{3}$ on/in $\mathrm{H}_{2} \mathrm{O}$ clusters: Structure, dynamics and spectroscopy" (unpublished).

Ball, P., 2004, "The joy of six: the growth and form of snow crystals," Interdiscipl. Sci. Rev. 29, 353.

Bamber, J., 2007, "Remote Sensing in Glaciology," in Glacier Science and Environmental Change, edited by P.G. Knight (Blackwell Publishing, Malden, MA), 1st ed., p. 370.

Bardeen, C. G., O. B. Toon, E. J. Jensen, D. R. Marsh, and V. L. Harvey, 2008, "Numerical simulations of the three-dimensional distribution of meteoric dust in the mesosphere and upper stratosphere," J. Geophys. Res. Atmos. 113, D17202.

Barkume, K. M., M.E. Brown, and E.L. Schaller, 2008, "Nearinfrared spectra of Centaurs and Kuiper Belt Objects," Astron. J. 135, 55 .

Bar-Nun, A., G. Herman, D. Laufer, and M. L. Rappaport, 1985, "Trapping and release of gases by water ice and implications for icy bodies," Icarus 63, 317.

Bar-Nun, A., G. Notesco, and T. Owen, 2007, "Trapping of $\mathrm{N}_{2}$, CO and Ar in amorphous ice-application to comets," Icarus 190, 655.

Bartels-Rausch, T., M. Brigante, Y. Elshorbany, M. Ammann, B. D'anna, C. George, K. Stemmler, M. Ndour, and J. Kleffmann, 2010, "Humic acid in ice: Photo-enhanced conversion of nitrogen dioxide into nitrous acid," Atmos. Environ. 44, 5443.

Bartels-Rausch, T., G. Krysztofiak, A. Bernhard, M. Schläppi, M. Schwikowski, and M. Ammann, 2011, "Photoinduced reduction of divalent mercury in ice by organic matter," Chemosphere 82, 199.

Bell, R. E., et al., 2011, "Widespread persistent thickening of the East Antarctic ice sheet by freezing from the base," Science 331, 1592.

Bell, III, J.F., 2009, "Water on Planets," Proceedings of the International Astronomical Union, Vol. 15, p. 29 [http:// dx.doi.org/10.1017/S1743921310008161].

Benatov, L., and J.S. Wettlaufer, 2004, "Abrupt grain boundary melting in ice," Phys. Rev. E 70, 061606.

Bergeron, V., C. Berger, and M.D. Betterton, 2006, "Controlled irradiative formation of penitentes,” Phys. Rev. Lett. 96, 098502.

Bernstein, M., 2006, "Prebiotic materials from on and off the early Earth,” Phil. Trans. R. Soc. B 361, 1689.

Bernstein, M. P., D. P. Cruikshank, and S. A. Sandford, 2005, "Near infrared laboratory spectra of solid $\mathrm{H}_{2} \mathrm{O} / \mathrm{CO}_{2}$ and $\mathrm{CH}_{3} \mathrm{OH} / \mathrm{CO}_{2}$ ice mixtures," Icarus 179, 527. 
Bernstein, M. P., D. P. Cruikshank, and S. A. Sandford, 2006, "Nearinfrared spectra of laboratory $\mathrm{H}_{2} \mathrm{OCH}_{4}$ ice mixtures," Icarus 181, 302.

Bertaux, J.-L., et al., 2007, “A warm layer in Venus' cryosphere and high-altitude measurements of $\mathrm{HF}, \mathrm{HCl}, \mathrm{H}_{2} \mathrm{O}$ and $\mathrm{HDO}$, " Nature (London) 450, 646.

Betterton, M.D., 2001, "Theory of structure formation in snowfields motivated by penitentes, suncups, and dirt cones," Phys. Rev. E 63, 056129.

Bibring, J.-P., et al., and OMEGA team, 2004, "Perennial water ice identified in the south polar cap of Mars," Nature (London) 428, 627.

Bina, C.R., and A. Navrotsky, 2000, "Possible presence of high-pressure ice in cold conducting slabs," Nature (London) 408, 844.

Bitz, C.M., M.M. Holland, M. Eby, and A.J. Weaver, 2001, "Simulating the ice-thickness distribution in a coupled climate model," J. Geophys. Res. 106, 2441.

Black, G. J., D. B. Campbell, and J.K. Harmon, 2010, "Radar measurements of Mercury's north pole at $70 \mathrm{~cm}$ wavelength," Icarus 209, 224.

Blake, D. F., L. J. Allamandola, S. Sandford, and F. Freund, 1991, "Clathrate type II hydrate formation in vacuo under astrophysical conditions," Meteoritics 26, 319 [http://adsabs.harvard.edu/abs/ 1991Metic..26R.319B].

Blunier, T., G. Floch, H.-W. Jacobi, and E. Quansah, 2005, "Isotopic view on nitrate loss in Antarctic surface snow," Geophys. Res. Lett. 32, L13501.

Boogert, A. C. A., and P. Ehrenfreund, 2004, "Interstellar ices," in Astrophysics of Dust, ASP Conference Series, edited by A. N. Witt, G. C. Clayton, and B. T. Draine (Astronomical Society of the Pacific, Estes Park, CO), Vol. 309, p. 547.

Boogert, A.C.A., et al., 2008, "The c2d spitzer spectroscopic survey of ices around low-mass young stellar objects. I. $\mathrm{H}_{2} \mathrm{O}$ and the 5-8 $\mu \mathrm{m}$ bands," Astrophys. J. 678, 985.

Boogert, A. C. A., W. A. Schutte, F. P. Helmich, A. G. G. M. Tielens, and D.H. Wooden, 1997, "Infrared observations and laboratory simulations of interstellar $\mathrm{CH}_{4}$ and $\mathrm{SO}_{2}$," Astron. Astrophys. 317, 929B.

Booth, G. K., 1999, "William Robertson Smith: The Scientific, Literary and Cultural Context from 1866 to 1881," Ph.D. thesis (University of Aberdeen).

Bowron, D. T., J. L. Finney, A. Hallbrucker, I. Kohl, T. Loerting, E. Mayer, and A. K. Soper, 2006, "The local and intermediate range structures of the five amorphous ices at $80 \mathrm{~K}$ and ambient pressure: A Faber-Ziman and Bhatia-Thornton analysis," J. Chem. Phys. 125, 194502.

Bronshteyn, V. K., and A. A. Chernov, 1991, "Freezing potentials arising on solidification of dilute aqueous solutions of electrolytes," J. Cryst. Growth 112, 129.

Brooke, T. Y., R. F. Knacke, Th. Encrenaz, P. Drossart, D. Crisp, and H. Feuchtgruber, 1998, "Models of the ISO 3- $\mu$ m Reflection Spectrum of Jupiter," Icarus 136, 1.

Broughton, J. Q., and G. H. Gilmer, 1986, "Thermodynamic criteria for grain-boundary melting — a molecular-dynamics study," Phys. Rev. Lett. 56, 2692.

Brown, D. E., S. M. George, C. Huang, E. K. L. Wong, K. B. Rider, R. S. Smith, and B. D. Kay, 1996, " $\mathrm{H}_{2} \mathrm{O}$ condensation coefficient and refractive index for vapor-deposited ice from molecular beam and optical interference measurements," J. Phys. Chem. 100, 4988.

Brown, M. E., D. Ragozzine, J. Stansberry, and W. C. Fraser, 2010, "The size, density, and formation of the Orcus-Vanth system in the Kuiper belt," Astron. J. 139, 2700.
Brown, R. H., D. P. Cruikshank, J. Veverka, P. Helfenstein, and J. Eluszkiewicz, 1995, Neptune and Triton (University of Arizona Press, Tucson).

Brownlee, D., et al., 2006, "Comet 81P/Wild 2 under a microscope," Science 314, 1711.

Buch, V., J.P. Devlin, I. Abrrey Monreal, B. Jagoda-Cwiklik, N. Uras-Aytemiz, and L. Cwiklik, 2009, "Clathrate hydrates with hydrogen-bonding guests," Phys. Chem. Chem. Phys. 11, 10245.

Buch, V., A. Dubrovskiy, F. Mohamed, M. Parrinello, J. Sadlej, A. D. Hammerich, and J. P. Devlin, 2008, " $\mathrm{HCl}$ hydrates as model systems for protonated water," J. Phys. Chem. A 112, 2144.

Buch, V., F. Mohamed, M. Parrinello, and J.P. Devlin, 2007, "Elusive structure of $\mathrm{HCl}$ monohydrate," J. Chem. Phys. 126, 074503.

Buch, V., J. Sadlej, N. Aytemiz-Uras, and J.P. Devlin, 2002, "Solvation and ionization stages of $\mathrm{HCl}$ on ice nanocrystals," J. Phys. Chem. A 106, 9374.

Bukowski, R., K. Szalewicz, G. C. Groenenboom, and A. van der Avoird, 2007, "Predictions of the properties of water from first principles," Science 315, 1249.

Burke, D. J., and W. A. Brown, 2010, "Ice in space: surface science investigations of the thermal desorption of model interstellar ices on dust grain analogue surfaces," Phys. Chem. Chem. Phys. 12, 5947.

Burniston, D. A., W. J. M. Strachan, J. T. Hoff, and F. Wania, 2007, "Changes in surface area and concentrations of semivolatile organic contaminants in aging snow," Environ. Sci. Technol. 41, 4932.

Burton, E. F., and W. F. Oliver, 1935, "The crystal structure of ice at low temperatures," Proc. R. Soc. A 153, 166.

Cahoy, K. L., M. S. Marley, and J. J. Fortney, 2010, "Exoplanet albedo spectra and colors as a function of planet phase, separation, and metallicity," Astrophys. J. 724, 189.

Callaghan, R., I. J. Lim, D.E. Murdock, J.J. Sloan, and D. J. Donaldson, 1994, "Laboratory simulation of polar stratospheric clouds," Geophys. Res. Lett. 21, 373.

Calvin, W. M., R. N. Clark, R.H. Brown, and J.R. Spencer, 1995, "Spectra of the icy Galilean satellites from 0.2 to $5 \mu \mathrm{m}$ : A compilation, new observations, and a recent summary," J. Geophys. Res. 100, 19041.

Campins, H., K. Hargrove, N. Pinilla-Alonso, E. S. Howell, M. S. Kelley, J. Licandro, T. Mothé-Diniz, Y. Fernández, and J. Ziffer, 2010, "Water ice and organics on the surface of the asteroid 24 Themis," Nature (London) 464, 1320.

Cantrell, W., and A. Heymsfield, 2005, "Production of ice in tropospheric clouds: A review," Bull. Am. Meteorol. Soc. 86, 795.

Capria, M. T., 2000, "Sublimation mechanisms of comet nuclei," Earth, Moon, Planets 89, 161.

Car, R., and M. Parrinello, 1985, "Unified approach for molecular dynamics and density-functional theory," Phys. Rev. Lett. 55, 2471.

Carlson, R. W., et al., 1999, "Hydrogen peroxide on the surface of Europa," Science 283, 2062.

Cartwright, J.H.E., 2007, "Ice in the solar system," in Solar and Stellar Physics Through Eclipses, ASP Conference Series, edited by O. Demircan, S. O. Selam, and B. Albayrak (Astronomical Society of the Pacific, Estes Park, CO), Vol. 370, p. 265.

Cartwright, J. H.E., B. Escribano, H. Grothe, O. Piro, C. I. SainzDíaz, and I. Tuval, 2011, "Nonlinear dynamics of ice growth and charge production in thunderstorms" (unpublished).

Cartwright, J. H.E., B. Escribano, and C. I. Sainz-Díaz, 2008, “The mesoscale morphologies of ice films: Porous and biomorphic 
forms of ice under astrophysical conditions," Astrophys. J. 687, 1406.

Cartwright, J. H. E., B. Escribano, and C. I. Sainz-Díaz, 2010, "Ice films follow structure zone model morphologies," Thin Solid Films 518, 3422.

Chaban, G. M., M. Bernstein, and D. P. Cruikshank, 2007, "Carbon dioxide on planetary bodies: Theoretical and experimental studies of molecular complexes," Icarus 187, 592.

Chen, A.S.-H., and S.W. Morris, 2011, "Experiments on the morphology of icicles," Phys. Rev. E 83, 026307.

Chiar, J. E., A. J. Adamson, T. H. Kerr, and D. C. B. Whittet, 1994, "Solid carbon monoxide in the Serpens dark cloud," Astrophys. J. 426, 240.

Chu, L., and C. Anastasio, 2005, "Formation of hydroxyl radical from the photolysis of frozen hydrogen peroxide," J. Phys. Chem. A 109, 6264.

Chyba, C. F., 2000, "Energy for microbial life on Europa," Nature (London) 403, 381.

Clary, D. C., and L. C. Wang, 1997, "Influence of surface defects on the adsorption of $\mathrm{HCl}$ on ice," J. Chem. Soc., Faraday Trans. 93, 2763.

Cochran, A. L., 2002, "A search for $\mathrm{N}^{2+}$ in spectra of comet $\mathrm{C} / 2002$ C1 (Ikeya-Zhang)," Astrophys. J. 576, L165.

Cochran, A. L., W. D. Cochran, and E. S. Barker, 2000, " $\mathrm{N}^{2+}$ and $\mathrm{CO}^{+}$in comets 122P/1995 S1 (deVico) and C/1995 O1 (HaleBopp)," Icarus 146, 583.

Colaprete, A., et al., 2010, "Detection of water in the LCROSS ejecta plume," Science 330, 463.

Collings, M. P., M. A. Anderson, R. Chen, J. W. Dever, S. Viti, D. A. Williams, and M. R. S. McCoustra, 2004, "A laboratory survey of the thermal desorption of astrophysically relevant molecules," Mon. Not. R. Astron. Soc. 354, 1133.

Collings, M. P., R. Chen, and M. R. S. McCoustra, 2006, "Probing the norphology of interstellar ice analogues," in Astrochemistry: From Laboratory Studies to Astronomical Observations, edited by R. I. Kaiser, P. Bernath, Y. Osamura, S. Petrie, and A. M. Mebel, AIP Conference Proceedings (AIP, New York), Vol. 855, p. 62.

Collings, M. P., J. W. Dever, H. J. Fraser, and M. R. S. McCoustra, 2003, "Laboratory studies of the interaction of carbon monoxide with water ice," Astrophys. Space Sci. 285, 633.

Comiso, J. C., C.L. Parkinson, R. Gersten, and L. Stock, 2008, "Accelerated decline in the Arctic sea ice cover," Geophys. Res. Lett. 35, L01703.

Cooper, J.F., R.E. Johnson, B.H. Mauk, H.B. Garret, and N. Gehrels, 2001, "Energetic ion and electron irradiation of the icy galilean satellites," Icarus 149, 133.

Corripio, J.G., 2003, "Modelling the energy balance of high altitude glacierised basins in the Central Andes," Ph.D. thesis (University of Edinburgh).

Corripio, J. G., and R. S. Purves, 2005, "Surface energy balance of high altitude glaciers in the central Andes: the effect of snow penitentes," in Climate and Hydrology in Mountain Areas, edited by C. de Jong, D. Collins, and R. Ranzi (Wiley, New York).

Coustenis, A., S. Atreya, P. Casavecchia, J. Castillo, O. Dutuit, H. Hussmann, and F. Sohl, 2008, "Surfaces and atmospheres of the outer planets, their satellites and ring systems: Part IV," Planet. Space Sci. 56, 1571.

Crawford, J. H., et al., 2001, "Evidence for photochemical production of ozone at the South Pole surface," Geophys. Res. Lett. 28, 3641 .

Creighan, S. C., J. S. A. Perry, and S. D. Price, 2006, “The rovibrational distribution of $\mathrm{H}_{2}$ and $\mathrm{HD}$ formed on a graphite surface at 15-50 K," J. Chem. Phys. 124, 114701.
Crovisier, J., 2005, "Cometary diversity and cometary families," in Proceedings of the XVIIIemes Rencontres de Blois, arXiv:0703785v1.

Crowley, J. N., M. Ammann, R. A. Cox, R. G. Hynes, M. E. Jenkin, A. Mellouki, M. J. Rossi, J. Troe, and T. J. Wallington, 2010, "Evaluated kinetic and photochemical data for atmospheric chemistry: Volume V-heterogeneous reactions on solid substrates," Atmos. Chem. Phys. 10, 9059.

Cruikshank, D.P., 2010, "Generating an Atmosphere," Science 330, 1755.

Cruikshank, D. P., R.H. Brown, W.M. Calvin, T.L. Roush, and M. J. Bartholomew, 1998, "Ices on the Satellites of Jupiter, Saturn and Uranus," Solar System Ices, ASSL Series (Kluwer Academic Publishers, Dordrecht), Vol. 227.

Cruikshank, D. P., J. Veverka, and L. A. Lebofsky, 1984, "Satellites of Saturn: the optical properties," in Saturn (University of Arizona Press, Tucson).

Cull, S., R.E. Arvidson, M. T. Mellon, P. Skemer, A. Shaw, and R. V. Morris, 2010, "Compositions of subsurface ices at the Mars Phoenix landing site," Geophys. Res. Lett. 37, 24203.

Cuppen, H. M., and E. Herbst, 2007, "Simulation of the formation and morphology of ice mantles on interstellar grains," Astrophys. J. 668, 294.

Cuppen, H. M., S. Ioppolo, C. Romanzin, and H. Linnartz, 2010, "Water formation at low temperatures by surface $\mathrm{O}_{2}$ hydrogenation II: the reaction network," Phys. Chem. Chem. Phys. 12, 12077.

Curry, J. A., J. L. Schramm, and E. E. Ebert, 1995, "Sea ice-albedo climate feedback mechanism, J. Clim. 8, 240.

Curtius, J., et al., 2005, "Observations of meteoric material and implications for aerosol nucleation in the winter Arctic lower stratosphere derived from in situ particle measurements," Atmos. Chem. Phys. 5, 3053.

Cuzzi, J., R. Clark, G. Filacchione, R. French, R. Johnson, E. Marouf, and L. Spilker, 2009, "Ring particle composition and size distribution," in Saturn from Cassini-Huygens, edited by M. K. Dougherty, L. W. Esposito, and S. M. Krimigis (Springer, New York), Chap. 15.

Dartois, E., and D. Deboffle, 2008, "Methane clathrate hydrate FTIR spectrum. Implications for its cometary and planetary detection," Astron. Astrophys. 490, L19.

Dartois, E., K. Demyk, L. D'Hendecourt, and P. Ehrenfreund, 1999, "Carbon dioxide-methanol intermolecular complexes in interstellar grain mantles," Astron. Astrophys. 351, 1066D.

Dartois, E., W. Schutte, T. R. Geballe, K. Demyk, P. Ehrenfreund, and L. D'Hendecourt, 1999, "Methanol: The second most abundant ice species towards the high-mass protostars RAFGL7009S and W 33A," Astron. Astrophys. 342L, 32D.

Dasgupta, P. K., and Y. Mo, 1997, "Chromatography on water-ice," Anal. Chem. 69, 4079.

Dash, J. G., A. W. Rempel, and J. S. Wettlaufer, 2006, "The physics of premelted ice and its geophysical consequences," Rev. Mod. Phys. 78, 695.

Dash, J. G., and J. S. Wettlaufer, 2003, "The surface physics of ice in thunderstorms," Can. J. Phys. 81, 201.

Davies, J. K., T. L. Roush, D. P. Cruikshank, M. J. Bartholomew, T. R. Geballe, T. Owen, and C. de Bergh, 1997, "The detection of water ice in comet Hale-Bopp," Icarus 127, 238.

Davies, P., 2003, The Origin of Life (Penguin, Toronto).

Debenedetti, P. G., 1996, Metastable Liquids (Princeton University Press, Princeton, NJ).

Debenedetti, P. G., 2003, "Supercooled and glassy water," J. Phys. Condens. Matter 15, R1669. 
Delsemme, A. H., and P. Swings, 1952, "Hydrates de gaz dans les noyaux cométaires et les grains interstellaires," Annales d'Astrophysique $\quad \mathbf{1 5}, \quad 1 \quad$ [http://adsabs.harvard.edu/full/ 1952AnAp...15...1D].

Demirdjian, B., D. Ferry, J. Suzanne, C. Toubin, S. Picaud, P. N. M. Hoang, and C. Girardet, 2002, "Structure and dynamics of ice Ih films upon $\mathrm{HCl}$ adsorption between 190 and 270 K. I. Neutron diffraction and quasielastic neutron scattering experiments," J. Chem. Phys. 116, 5143.

DeMott， P. J., D. J. Cziczo，A. J. Prenni， D. M. Murphy, S. M. Kreidenweis, D. S. Thomson, R. Borys, and D. C. Rogers, 2003, "Measurements of the concentration and composition of nuclei for cirrus formation," Proc. Natl. Acad. Sci. U.S.A. 100, 14655.

DeMott, P. J., A. J. Prenni, X. Liu, S. M. Kreidenweis, M. D. Petters, C. H. Twohy, M. S. Richardson, T. Eidhammer, and D. C. Rogers, 2010, "Predicting global atmospheric ice nuclei distributions and their impacts on climate," Proc. Natl. Acad. Sci. U.S.A. 107, 11217.

Devlin, J. P., N. Uras, J. Sadlej, and V. Buch, 2002, “Discrete stages in the solvation and ionization of hydrogen chloride adsorbed on ice particles," Nature (London) 417, 269.

Doble, M. J., M. D. Coon, and P. Wadhams, 2003, "Pancake ice formation in the Weddell Sea," J. Geophys. Res. 108, 3209.

Dohnálek, Z., R. L. Ciolli, G. A. Kimmel, K. P. Stevenson, R. S. Smith, and B.D. Kay, 1999, "Substrate induced crystallization of amorphous solid water at low temperatures," J. Chem. Phys. 110, 5489.

Dohnálek, Z., G. A. Kimmel, R. L. Ciolli, K. P. Stevenson, R. S. Smith, and B.D. Kay, 2000, "The effect of the underlying substrate on the crystallization kinetics of dense amorphous solid water films," J. Chem. Phys. 112, 5932.

Dominé, F., M. Albert, T. Huthwelker, H.-W. Jacobi, A. A. Kokhanovsky, M. Lehning, G. Picard, and W. R. Simpson, 2008, "Snow physics as relevant to snow photochemistry," Atmos. Chem. Phys. 8, 171.

Dominé, F., and P. Shepson, 2002, "Air-snow interactions and atmospheric chemistry," Science 297, 1506.

Dotto, E., M. A. Barucci, and C. de Bergh, 2003, "Surface composition of TNOs and Centaurs: visible and near-infrared spectroscopy," C.R. Physique 4, 775.

Drews, R., O. Eisen, I. Weikusat, S. Kipfstuhl, A. Lambrecht, D. Steinhage, F. Wilhelms, and H. Miller, 2009, "Layer disturbances and the radio-echo free zone in ice sheets," Cryosphere 3, 195.

Duft, D., and T. Leisner, 2004, "Laboratory evidence for volumedominated nucleation of ice in supercooled water microdroplets," Atmos. Chem. Phys. 4, 1997.

Dulieu, F., L. Amiaud, E. Congiu, J.H. Fillion, E. Matar, A. Momeni, V. Pirronello, and J. L. Lemaire, 2010, "Experimental evidence for water formation on interstellar dust grains by hydrogen and oxygen atoms," Astron. Astrophys. 512, A30.

Durant, A.J., and R.A. Shaw, 2005, "Evaporation freezing by contact nucleation inside-out," Geophys. Res. Lett. 32, L20814.

Durham, W. B., and L. A. Stern, 2001, "Rheological properties of water ice-applications to satellites of the outer planets," Annu. Rev. Earth Planet. Sci. 29, 295.

Ehrenfreund, P., L. d'Hendecourt, S. B. Charnley, and R. Ruiterkamp, 2001, "Energetic and thermal processing of interstellar ices,” J. Geophys. Res. 106, 33291.

Ehrenfreund, P., H.J. Fraser, J. Blum, J.H.E. Cartwright, J. M. García-Ruiz, E. Hadamcik, A.C. Levasseur-Regourd, S. Price, F. Prodi, and A. Sarkissian, 2003, "Physics and chemistry of icy particles in the universe: Answers from microgravity," Planet. Space Sci. 51, 473.
Eigen, M., and P. Schuster, 1979, The Hypercycle-A Principle of Natural Self-Organization (Springer, Berlin).

Ekström, M., P. Eriksson, W. G. Read, M. Milz, and D. P. Murtagh, 2008, "Comparison of satellite limb-sounding humidity climatologies of the uppermost tropical troposphere," Atmos. Chem. Phys. 8, 309.

Elbaum, M., S. G. Lipson, and J. G. Dash, 1993, "Optical study of surface melting on ice," J. Cryst. Growth 129, 491.

Elbaum, M., and M. Schick, 1991, "Application of the theory of dispersion forces to the surface melting of ice," Phys. Rev. Lett. 66, 1713.

Engemann, S., H. Reichert, H. Dosch, J. Bilgram, V. Honkimäki, and A. Snigirev, 2004, "Interfacial melting of ice in contact with $\mathrm{SiO}_{2}$," Phys. Rev. Lett., 92, 205701.

Escribano, R., D. Fernández-Torre, V. J. Herrero, B. MartínLlorente, B. Maté, I. K. Ortega, and H. Grothe, 2007, "The low-frequency Raman and IR spectra of nitric acid hydrates," Vib. Spectrosc. 43, 254.

Estrin, D. A., J. Kohanoff, D.H. Laria, and R. O. Weht, 1997, "Hybrid quantum and classical mechanical Monte Carlo simulations of the interaction of hydrogen chloride with solid water clusters," Chem. Phys. Lett. 280, 280.

Falenty, A., G. Genov, T.C. Hansen, W.F. Kuhs, and A. N. Salamatin, 2011, "Kinetics of $\mathrm{CO}_{2}$ hydrate formation from water frost at low temperatures: Experimental results and theoretical model," J. Phys. Chem. C 115, 4022.

Fanfoni, M., and M. Tomellini, 1998, "The Johnson-Mehl-AvramiKolmogorov model: A brief review," Nuovo Cimento D 20, 1171.

Faria, S. H., S. Kipfstuhl, N. Azuma, J. Freitag, I. Weikusat, M. M. Murshed, and W.F. Kuhs, 2009, "Multiscale Structures in the Antarctic Ice Sheet Part I: Inland Ice," Physics of Ice Core Records II, edited by T.H. Sapporo, Low Temperature Science, Supplement Issue 68 (Institute of Low Temperature Science, Hokkaido University, Japan) [http://hdl.handle.net/ 2115/45430].

Fedorova, A., et al., 2008, "HDO and $\mathrm{H}_{2} \mathrm{O}$ vertical distributions and isotopic ratio in the Venus mesosphere by Solar Occultation at Infrared spectrometer on board Venus Express,' J. Geophys. Res. 113, E00B22.

Feltham, D. L., 2008, “Sea ice rheology,” Annu. Rev. Fluid Mech. 40, 91.

Feltham, D. L., N. Untersteiner, J. S. Wettlaufer, and M. G. Worster, 2006, "Sea ice is a mushy layer," Geophys. Res. Lett. 33, L14501.

Ferris, J. P., 1998, "Catalyzed RNA synthesis for the RNA world," in The Molecular Origins of Life, edited by A. Brack (Cambridge University Press, Cambridge, England) p. 255.

Feynman, R.P., 1967, The Character of Physical Law: The 1964 Messenger Lectures (MIT Press, Cambridge, MA).

Finney, J. L., 2001, “Ice: Structures," in Encyclopedia of Materials: Science and Technology, edited by K. H. J. Buschow, R. W. Cahn, M. C. Flemings, B. Ilschner, E. J. Kramer, and S. Mahajan (Elsevier Science, Oxford), Vol. 5, p. 4018.

Finney, J.L., 2004, "Ice: the laboratory in your freezer", Interdiscipl. Sci. Rev. 29, 339.

Finney, J. L., D. T. Bowron, A. K. Soper, T. Loerting, E. Mayer, and A. Hallbrucker, 2002, "Structure of a new dense amorphous ice," Phys. Rev. Lett. 89, 205503.

Finney, J.L., A. Hallbrucker, I. Kohl, A. K. Soper, and D. T. Bowron, 2002, "Structures of high and low density amorphous ice by neutron diffraction," Phys. Rev. Lett. 88, 225503.

Firanescu, G., D. Hermsdorf, R. Ueberschaer, and R. Signorell, 2006, "Large molecular aggregates: from atmospheric aerosols to drug nanoparticles," Phys. Chem. Chem. Phys. 8, 4149. 
Flammer, K. R., D. A. Mendis, and H. L. F. Houpis, 1998, "On the outgassing profile of comet Hale-Bopp," Astrophys. J. 494, 822.

Fogle, B., and B. Haurwitz, 1966, "Noctilucent clouds," Space Sci. Rev. 6, 279.

Fraser, H.J., M.P. Collings, and M.R.S. McCoustra, 2002, "Laboratory surface astrophysics experiment," Rev. Sci. Instrum. 73, 2161.

French, N. H. F., E. S. Kasischke, L. L. Bourgeau-Chavez, and P. A. Harrell, 1996, "Monitoring variations in soil moisture on fire disturbed sites in alaska using ERS-1 SAR imagery," Int. J. Remote Sensing 17, 3037.

French, R. H., et al., 2010, "Long range interactions in nanoscale science," Rev. Mod. Phys. 82, 1887.

Fukazawa, H., A. Hoshikawa, H. Yamauchi, Y. Yamaguchi, N. Igawa, and Y. Ishiib, 2006, "Deuteron ordering in ice containing impurities: A neutron diffraction study," Physica (Amsterdam) 385-386B, 113.

Fukazawa, H., A. Hoshikawa, H. Yamauchi, Y. Yamaguchi, and Y. Ishiib, 2005, "Formation and growth of ice XI: A powder neutron diffraction study," J. Cryst. Growth 282, 251.

Furukawa, Y., and H. Nada, 1997a, "Anisotropic surface melting of an ice crystal and its relationship to growth forms," J. Phys. Chem. B 101, 6167.

Furukawa, Y., and H. Nada, 1997b, "Anisotropy in microscopic structures of ice-water and ice-vapor interfaces and its relation to growth kinetics," in Advances in the Understanding of Crystal Growth Mechanisms, edited by T. Nishinaga, K. Nishioka, J. Harada, A. Sasaki, and H. Takei (Elsevier, New York), p. 559.

Gálvez, O., I. K. Ortega, B. Maté, M. A. Moreno, B. MartínLlorente, V. J. Herrero, R. Escribano, and P. J. Gutiérrez, 2007, "A study of the interaction of $\mathrm{CO}_{2}$ with water ice," Astron. Astrophys. 472, 691.

Gao, R. S., et al., 2004, "Evidence that nitric acid increases relative humidity in low-temperature cirrus clouds," Science 303, 516.

Gautier, D., and F. Hersant, 2005, "Formation and composition of planetesimals," Space Sci. Rev. 116, 25.

Gerakines, P. A., J. J. Bray, A. Davis, and C. R. Richey, 2005, "The strengths of near-infrared absorption features relevant to interstellar and planetary ices," Astrophys. J. 620, 1140.

Gerakines, P. A., et al., 1999, "Observations of solid carbon dioxide in molecular clouds with the infrared space observatory," Astrophys. J. 522, 357.

Gibb, E.L., et al., 2000, "An inventory of interstellar ices toward the embedded protostar W33A," Astrophys. J. 536, 347.

Gibson, K.D., D. R. Killelea, H.Q. Yuan, J. S. Becker, and S. J. Sibener, 2011, "Determination of the sticking coefficient and scattering dynamics of water on ice using molecular beam techniques," J. Chem. Phys. 134, 034703.

Gil-Hutton, R., J. Licandro, N. Pinilla-Alonso, and R. Brunetto, 2009, "The trans-Neptunian object size distribution at small sizes," Astron. Astrophys. 500, 909.

Girard, L., S. Bouillon, J. Weiss, D. Amitrano, T. Fichefet, and V. Legat, 2011, "A new modelling framework for sea-ice mechanics based on elasto-brittle rheology," Annals of Glaciology 52, 123.

Givan, A., H. Grothe, A. Loewenschuss, and C. Nielsen, 2002, "Infrared spectra and ab initio calculations of matrix isolated dimethyl sulfone and its water complex," Phys. Chem. Chem. Phys. 4, 255.

Goedecker, S., M. Teter, and J. Hutter, 1996, "Separable dual-space gaussian pseudopotentials,” Phys. Rev. B 54, 1703.
Gómez, P.C., O. Gálvez, and R. Escribano, 2009, “Theoretical study of atmospheric clusters: $\mathrm{HNO}_{3}-\mathrm{HCl}-\mathrm{H}_{2} \mathrm{O}$," Phys. Chem. Chem. Phys. 11, 9710.

Gómez, P.C., O. Gálvez, R.G. Mosteo, C. Puzzarini, and R. Escribano, 2010, "Clusters of atmospheric relevance: $\mathrm{H}_{2} \mathrm{O} / \mathrm{HCl} / \mathrm{HNO}_{3}$. Prediction of IR \& MW spectra," Phys. Chem. Chem. Phys. 12, 4617.

Gomis, O., M.A. Satorre, G. Strazzulla, and G. Leto, 2004, "Hydrogen peroxide formation by ion implantation in water ice and its relevance to the galilean satellites," Planet. Space Sci. 52, 371.

González, M., P. J. Gutiérrez, L. M. Lara, and R. Rodrigo, 2008, "Evolution of the crystallization front in cometary models. Effect of the net energy released during crystallization," Astron. Astrophys. 486, 331.

Graham, J.D., and J.T. Roberts, 2000, "Chemical reactions of organic molecules adsorbed at ice: 2. Chloride substitution in 2-methyl-2-propanol," Langmuir 16, 3244.

Grannas, A., A. Bausch, and K. Mahanna, 2007, "Enhanced aqueous photochemical reaction rates after freezing," J. Phys. Chem. A 111, 11043.

Grannas, A. M., et al., 2007, "An overview of snow photochemistry: Evidence, mechanisms and impacts," Atmos. Chem. Phys. 7, 4329.

Grannas, A.M., P.B. Shepson, and T.R. Filley, 2004, "Photochemistry and nature of organic matter in Arctic and Antarctic snow," Global Biogeochem Cycles 18, GB1006.

Graversen, R. G., T. Mauritsen, M. Tjernström, E. Källen, and G. Svensson, 2008, "Vertical structure of recent Arctic warming," Nature (London) 451, 53.

Greve, R., 2006, "Fluid dynamics of planetary ices," GAMM-Mitteilungen 29, 29 [http://arxiv.org/pdf/0903.3773.pdf].

Grothe, H., 2008, Referee comment on "Inhibition of ice crystallization in highly viscous aqueous organic acid droplets," by B. J. Murray, Atmos. Chem. Phys. Discuss. 8, S3992 [www .atmos-chem-phys-discuss.net/8/S3992/2008/].

Grothe, H., C.E. Lund Myhre, and C. J. Nielsen, 2006, Lowfrequency Raman spectra of nitric acid hydrates," J. Phys. Chem. A 110, 171.

Grothe, H., H. Tizek, D. Waller, and D. J. Stokes, 2006, The crystallization kinetics and morphology of nitric acid trihydrate, Phys. Chem. Chem. Phys. 8, 2232.

Guillot, B., and Y. Guissani, 2003, "Polyamorphism in low temperature water: A simulation study, J. Chem. Phys. 119, 11740.

Gumbel, J., and L. Megner, 2009, "Charged meteoric smoke as ice nuclei in the mesosphere: Part 1-a review of basic concepts," J. Atmos. Sol. Terr. Phys. 71, 1225.

Gumbel, J., and G. Witt, 1998, "In situ measurements of the vertical structure of a noctilucent cloud," Geophys. Res. Lett. 25, 493.

Gurganus, C., A. B. Kostinski, and R. A. Shaw, 2011, "Fast imaging of freezing drops: No preference for nucleation at the contact line," J. Phys. Chem. Lett. 2, 1449.

Haapala, J., N. Lönnroth, and A. Stössel, 2005, "A numerical study of open water formation in sea ice," J. Geophys. Res. 110, C09011.

Haas, C., A. Pfaffling, S. Hendricks, L. Rabenstein, J.L. Etienne, and I. Rigor, 2008, "Reduced ice thickness in Arctic transpolar drift favors rapid ice retreat," Geophys. Res. Lett. 35, L17 501.

Haeberli, W., et al., 2006, "Permafrost creep and rock glacier dynamics," Permafrost Periglac. Process. 17, 189.

Hage, W., A. Hallbrucker, E. Mayer, and G. P. Johari, 1994, "Crystallization kinetics of water below $150 \mathrm{~K}$," J. Chem. Phys. 100, 2743. 
Hage, W., A. Hallbrucker, E. Mayer, and G.P. Johari, 1995, "Kinetics of crystallizing $\mathrm{D}_{2} \mathrm{O}$ water near $150 \mathrm{~K}$ by Fourier transform infrared spectroscopy and a comparison with the corresponding calorimetric studies on $\mathrm{H}_{2} \mathrm{O}$ water,' J. Chem. Phys. 103, 545.

Hale, B. N., and S. M. Kathmann, 1996, "Monte Carlo simulations of small $\mathrm{H}_{2} \mathrm{SO}_{4}-\mathrm{H}_{2} \mathrm{O}$ clusters," in Nucleation and Atmospheric Aerosols. Proceedings of the 14th International Conference (Pergamon, Oxford).

Hallquist, M., et al., 2009, "The formation, properties and impact of secondary organic aerosol: current and emerging issues," Atmos. Chem. Phys. 9, 5155.

Hancock, L. O., and H. Povenmire, 2010, "Earth: A ringed planet?," AGU Fall Meeting Abstracts, B1635+.

Handa, Y.P., D. D. Klug, and E. Whalley, 1986, "Difference in energy between cubic and hexagonal ice," J. Chem. Phys. 84, 7009.

Handa, Y. P., D. D. Klug, and E. Whalley, 1987, "Phase transitions of ice V and VI," J. Phys. (Paris), Colloq. 48, C1-435.

Hansen, J., M. Sato, P. Kharecha, G. Russell, D. Lea, and M. Siddall, 2007, "Climate change and trace gases," Phil. Trans. R. Soc. A 365, 1925.

Hansen, T. C., M. M. Koza, and W. F. Kuhs, 2008, "Formation and annealing of cubic ice: I. Modelling of stacking faults," J. Phys. Condens. Matter 20, 285104.

Hansen, T.C., M.M. Koza, P. Lindner, and W.F. Kuhs, 2008, "Formation and annealing of cubic ice: II. Kinetic study," J. Phys. Condens. Matter 20, 285105.

Hanson, D. R., and A. R. Ravishankara, 1991, "The reaction probabilities of $\mathrm{CLONO}_{2}$ and $\mathrm{N}_{2} \mathrm{O}_{5}$ on polar stratospheric cloud materials,” J. Geophys. Res. Atmos. 96, 5081.

Hanson, D. R., and A. R. Ravishankara, 1992, "Investigation of the reactive and nonreactive processes involving $\mathrm{ClONO}_{2}$ and $\mathrm{HCl}$ on water and nitric-acid doped ice," J. Phys. Chem. 96, 2682.

Hantal, G., P. Jedlovszky, P. N. M Hoang, and S. Picaud, 2008, "Investigation of the adsorption behaviour of acetone at the surface of ice. A grand canonical Monte Carlo simulation study," Phys. Chem. Chem. Phys. 10, 6369.

Harris, C., et al., 2009, "Permafrost and climate in Europe: Monitoring and modelling thermal, geomorphological and geotechnical responses," Earth Sci. Rev. 92, 117.

Hartmann, S., D. Niedermeier, J. Voigtlander, T. Clauss, R. A. Shaw, H. Wex, A. Kiselev, and F. Stratmann, 2011, "Homogeneous and heterogeneous ice nucleation at lacis: operating principle and theoretical studies," Atmos. Chem. Phys. 11, 1753.

Heißelmann, D., J. Blum, H.J. Fraser, and K. Wolling, 2010, "Microgravity experiments on the collisional behavior of saturnian ring particles," Icarus 206, 424.

Herman, A., 2011, "Molecular-dynamics simulation of clustering processes in sea-ice floes," Phys. Rev. E 84, 056104.

Heymsfield, A.J., 1986, "Ice particles observed in a cirriform cloud at $-83{ }^{\circ} \mathrm{C}$ and implications for polar stratospheric clouds," J. Atmos. Sci. 43, 851.

Heymsfield, A.J., and C.M.R. Platt, 1984, "A parameterization of the particle-size spectrum of ice clouds in terms of the ambienttemperature and the ice water-content," J. Atmos. Sci. 41, 846.

Hibbitts, C.A., and J. Szanyi, 2007, "Physisorption of $\mathrm{CO}_{2}$ on non-ice materials relevant to icy satellites," Icarus 191, 371.

Hibler, III, W.D., 2001, "Sea ice fracturing on the large scale," Eng. Fract. Mech. 68, 2013.

Hibler, III, W. D, 1979, "A dynamic thermodynamic sea ice model," J. Phys. Oceanogr. 9, 815.
Hidaka, H., M. Watanabe, A. Kouchi, and N. Watanabe, 2009, "Reaction routes in the $\mathrm{CO}-\mathrm{H}_{2} \mathrm{CO}-\mathrm{d}_{n}-\mathrm{CH}_{3} \mathrm{OH}-\mathrm{d}_{m}$ system clarified from $\mathrm{H}(\mathrm{D})$ exposure of solid formaldehyde at low temperatures, Astrophys. J. 702, 291.

Hillert, M., 1965, "On theory of normal and abnormal grain growth," Acta Metall. 13, 227.

Hiraoka, K., N. Mochizuki, and A. Wada, 2006, in Astrochemistry: From Laboratory Studies to Astronomical Observations, AIP Conference Proceedings No. 855, edited by R. I. Kaiser, P. Bernath, Y. Osamura, S. Petrie, and A. M. Mebel (AIP, Melville, NY), p. 86.

Hobbs, P. V., 1974, Ice Physics (Oxford University Press, Oxford), reprinted 2010.

Hodyss, R., P. V. Johnson, J. V. Stern, J. D. Goguen, and I. Kanik, 2009, "Photochemistry of methane water ices," Icarus 200, 338.

Hodyss, R., P. V. Jonson, G. E. Orzechowska, J. D. Goguen, and I. Kanik, 2008, "Carbon dioxide segregation in 1:4 and 1:9 $\mathrm{CO}_{2}$ : $\mathrm{H}_{2} \mathrm{O}$ ices," Icarus 194, 836.

Holland, M.M., and C.M. Bitz, 2003, "Polar amplification of climate change in the coupled model intercomparison project," Climate Dynamics 21, 221.

Holland, M. M., C. M. Bitz, E. C. Hunke, W. H. Lipscomb, and J. L. Schramm, 2006, "Influence of sea ice thickness distribution on polar climate in CCSM3,” J. Clim. 19, 2398.

Höpfner, M., et al., 2006, "Spectroscopic evidence for NAT, STS, and ice in MIPAS infrared limb emission measurements of polar stratospheric clouds," Atmos. Chem. Phys. 6, 1201.

Hopkins, M. A., 1998, "Four stages of pressure ridging," J. Geophys. Res. 103, 21883.

Hopkins, M. A., S. Frankenstein, and A. S. Thorndike, 2004, "Formation of an aggregate scale in Arctic sea ice," J. Geophys. Res. 109, C01032.

Hopkins, M. A., J. Tuhkuri, and M. Lensu, 1999, "Rafting and ridging of thin ice sheets," J. Geophys. Res. 104, 13605.

Hornekaer, L., A. Baurichter, V. V. Petrunin, D. Field, and A. C. Luntz, 2003, "Importance of surface morphology in interstellar $\mathrm{H}_{2}$ formation," Science 302, 1943.

Hornekaer, L., A. Baurichter, V. V. Petrunin, A. C. Luntz, B. D. Kay, and A. Al-Halab, 2005, "Influence of surface morphology on $\mathrm{D}_{2}$ desorption kinetics from amorphous solid water," J. Chem. Phys. 122, 124701.

Hornekaer, L., E. Rauls, W. Xu, R. Otero, Z. Sljivancanin, I. Stensgaard, E. Laegsgaard, B. Hammer, and F. Besenbacher, 2006, "Clustering of chemisorbed H(D) atoms on the graphite (0001) surface due to preferential sticking," Phys. Rev. Lett. 97, 186102.

Hornekaer, L., Z. Sljivancanin, W. Xu, R. Otero, E. Rauls, I. Stensgaard, E. Laegsgaard, B. Hammer, and F. Besenbacher, 2006, "Metastable structures and recombination pathways for atomic hydrogen on the graphite (0001) surface," Phys. Rev. Lett. 96, 156104.

Houpis, H. L. F., D. A. Mendis, and W.-H. Ip, 1985, “The chemical differentiation of the cometary nucleus-the process and its consequences," Astrophys. J. 295, 654.

Hudson, R. L., and B. Donn, 1991, "An experimental-study of the sublimation of water ice and the release of trapped gases," Icarus 94, 326.

Hughes, T., 2009, "Thermal convection and the origin of ice streams," J. Glaciol. 55, 524.

Huthwelker, T., M. Ammann, and T. Peter, 2006, "The uptake of acidic gases on ice," Chem. Rev. 106, 1375.

Huybrechts, P., 2007, "Numerical modelling of polar ice sheets through time," in Glacier Science and Environmental Change, 
edited by P. G. Knight (Blackwell Publishing, Malden, MA), 1st ed., p. 406.

Hvidegaard, S. M., R. Forsberg, and H. Skourup, 2006, "Sea ice thickness estimates from airborne laser scanning," in Arctic Sea Ice Thickness (Climate Change and Natural Hazards series 10), edited by P. Wadhams and G. Amanatidis (Office for Official Publication of the European Communities, Luxembourg), p. 193 [http://ec.europa.eu/research/environment/pdf/sea_ice_ thickness2006.pdf].

Imshenetsky, A. A., S. V. Lysenko, and G. A. Kazakov, 1978, "Upper boundary of the biosphere," Appl. Environ. Microbiol. 35, 1.

Ioppolo, S., H. M. Cuppen, C. Romanzin, E. F. van Dishoeck, and H. Linnartz, 2010, "Water formation at low temperatures by surface $\mathrm{O}_{2}$ hydrogenation I: characterization of ice penetration," Phys. Chem. Chem. Phys. 12, 12065.

Ioppolo, S., M.E. Palumbo, G. A. Baratta, and V. Mennella, 2009, "Formation of interstellar solid $\mathrm{CO}_{2}$ after energetic processing of icy grain mantles," Astron. Astrophys. 493, 1017.

Islam, F., E. R. Latimer, and S. D. Price, 2007, "The formation of vibrationally excited HD from atomic recombination on cold graphite surfaces," J. Chem. Phys. 127, 064701.

Jackson, S. M., V. M. Nield, R. W. Whitworth, M. Oguro, and C. C. Wilson, 1997, "Single-crystal neutron diffraction studies of the structure of ice XI," J. Phys. Chem. B 101, 6142.

Jenniskens, P., and D.F. Blake, 1994, "Structural transitions in amorphous water ice and astrophysical implications," Science 265, 753 .

Jenniskens, P., and D.F. Blake, 1996a, "Crystallization of amorphous water ice in the solar system," Astrophys. J. 473, 1104.

Jenniskens, P., and D. F. Blake, 1996b, "A mechanism for forming deep cracks in comets," Planet. Space Sci. 44, 711.

Jenniskens, P., D. F. Blake, M. A. Wilson, and A. Pohorille, 1995, "High-density amorphous ice, the frost on interstellar grains," Astrophys. J. 455, 389.

Jensen, E. J., et al., 2005, "Ice supersaturations exceeding 100\% at the cold tropical tropopause: implications for cirrus formation and dehydration," Atmos. Chem. Phys. 5, 851.

Johari, G. P., 1998, "On the coexistence of cubic and hexagonal ice between 160 and $240 \mathrm{~K}$," Philos. Mag. B 78, 375.

Johns, T. C., et al., 2006, "The new Hadley Centre climate model (HadGEM1): Evaluation of coupled simulations," J. Clim. 19, 1327.

Johnson, R. E., 1990, "Energetic charged-particle interaction with atmospheres and surfaces," in Physics and Chemistry in Space (Springer, New York), Vol. 19.

Kadosaki, G., T. Ichimaru, N. Hirasawa, and T. Yamanouchi, 2010, "The Information of PSC and PMC from GOSAT FTS," AGU Fall Meeting Abstracts, B210.

Kahan, T.F., R. Zhao, K. B. Jumaa, and D. J. Donaldson, 2010, "Anthracene photolysis in aqueous solution and ice: Photon flux dependence and comparison of kinetics in bulk ice and at the airice interface," Environ. Sci. Technol. 44, 1302.

Kaleschke, L., et al., 2004, "Frost flowers on sea ice as a source of sea salt and their influence on tropospheric halogen chemistry," Geophys. Res. Lett. 31, L16114.

Kärcher, B., J.P.D. Abbatt, R. A. Cox, P. J. Popp, and C. Voigt, 2009, "Trapping of trace gases by growing ice surfaces including surface-saturated adsorption," J. Geophys. Res. Atmos. 114, D13306.

Kargel, J.S., 2004, Mars-A Warmer Wetter Planet (Springer, New York), 1st ed.
Kaser, G., 2007, "Mountain glaciers," in Glacier Science and Environmental Change (Blackwell Publishing, Malden, MA), 1st ed., p. 268.

Kawada, S., 1972, "Dielectric dispersion and phase transition of KOH doped ice,” J. Phys. Soc. Jpn. 32, 1442.

Kawakita, H., J. Watanabe, T. Ootsubo, R. Nakamura, T. Fuse, N. Takato, S. Sasaki, and T. Sasaki, 2004, "Evidence of icy grains in comet C/2002 T7 (linear) at 3.52 AU," Astrophys. J. Lett. 601, L191.

Kerbrat, M., T. Huthwelker, H. Gäggeler, and M. Ammann, 2010, "Interaction of nitrous acid with polycrystalline ice: Adsorption on the surface and diffusion into the bulk," J. Phys. Chem. C 114, 2208.

Kerbrat, M., B. Pinzer, T. Huthwelker, H.W. Gäggeler, M. Ammann, and M. Schneebeli, 2008, "Measuring the specific surface area of snow with X-ray tomography and gas adsorption: comparison and implications for surface smoothness," Atmos. Chem. Phys. 8, 1261.

Kessler, M. A., and B. T. Werner, 2003, "Self-organization of sorted patterned ground," Science 299, 380.

Khalizov, A. F., M.E. Earle, W. J. W. Johnson, G. D. Stubley, and J. J. Sloan, 2006, "Development and characterization of a laminar aerosol flow tube,” Rev. Sci. Instrum. 77, 033102.

Kirner, O., R. Ruhnke, J. Buchholz-Dietsch, P. Jöckel, C. Brühl, and B. Steil, 2010, "Simulation of polar stratospheric clouds in the chemistry-climate-model EMAC via the submodel PSC," Geosci. Model Dev. Discuss. 3, 2071.

Klinger, J., 1981, "Some consequences of a phase transition of water ice on the heat balance of comet nuclei," Icarus 47, 320.

Knight, C., and S. J. Singer, 2006, "A re-examination of the ice III/ IX hydrogen bond ordering phase transition," J. Chem. Phys. 125, 064506.

Knopf, D. A., P.A. Alpert, B. Wang, and J. Y. Aller, 2011, "Stimulation of ice nucleation by marine diatoms," Nature Geosci. 4, 88.

Kobayashi, T., and T. Kuroda, 1987, "Snow crystals," in Morphology of Crystals, edited by I. Sunagawa (Terra Scientific Publishing, Tokyo) p. 649.

Kohl, I., E. Mayer, and A. Hallbrucker, 2000, “The glassy watercubic ice system: a comparative study by X-ray diffraction and differential scanning calorimetry, Phys. Chem. Chem. Phys. 2, 1579 .

Kohl, I., E. Mayer, and A. Hallbrucker, 2001, "Ice XII forms on compression of hexagonal ice at $77 \mathrm{~K}$ via high-density amorphous water," Phys. Chem. Chem. Phys. 3, 602.

Kolb, C.E., et al., 2010, "An overview of current issues in the uptake of atmospheric trace gases by aerosols and clouds," Atmos. Chem. Phys. 10, 10561.

Kong, X., P. U. Andersson, N. Marković, and J. B. C. Pettersson, 2011, "Environmental molecular beam studies of ice surface processes," Physics and Chemistry of Ice (to be published).

König, H., 1943, "Eine kubische Eismodifikation,” Z. Kristallogr. 105, 279.

Koop, T., 2004, "Homogeneous ice nucleation in water and aqueous solutions," Z. Phys. Chem. 218, 1231.

Koop, T., B. Luo, A. Tsias, and T. Peter, 2000, "Water activity as the determinant for homogeneous ice nucleation in aqueous solutions," Nature (London) 406, 611.

Korolev, A., G. A. Isaac, and J. Hallett, 2000, "Ice particle habits in stratiform clouds," Q. J. R. Meteorol. Soc. 126, 2873.

Kouchi, A., and S. Sirono, 2001, "Crystallization heat of impure amorphous $\mathrm{H}_{2} \mathrm{O}$ ice," Geophys. Res. Lett. 28, 827. 
Koza, M. M., 2009, “Transient pronounced density variation in amorphous ice structures," Z. Phys. Chem. 223, 979.

Koza, M. M., H. Schober, H.E. Fischer, T. Hansen, and F. Fujara, 2003, "Kinetics of the high- to low-density amorphous water transition,” J. Phys. Condens. Matter 15, 321.

Koza, M. M., H. Schober, T. Hansen, A. Tölle, and F. Fujara, 2000, "Ice XII in its second regime of metastability," Phys. Rev. Lett. 84, 4112.

Koza, M. M., H. Schober, A. Tölle, F. Fujara, and T. Hansen, 1999, "Formation of ice XII at different conditions," Nature (London) 397, 660 .

Krämer, M., et al., 2009, "Ice supersaturations and cirrus cloud crystal numbers," Atmos. Chem. Phys. 9, 3505.

Křepelová, A., J. Newberg, T. Huthwelker, H. Bluhm, and M. Ammann, 2010, "The nature of nitrate at the ice surface studied by XPS and NEXAFS," Phys. Chem. Chem. Phys. 12, 8870.

Kuhs, W.F., D. V. Bliss, and J.L. Finney, 1987, "High-resolution neutron powder diffraction study of ice Ic," J. Phys. (Paris), Colloq. 48, C1-631.

Kuhs, W.F., J.L. Finney, C. Vettier, and D. V. Bliss, 1984, "Structure and hydrogen ordering in ices VI, VII and VIII by neutron powder diffraction," J. Chem. Phys. 81, 3612.

Kuhs, W. F., G. Genov, D. K. Staykova, and T. Hansen, 2004, "Ice perfection and the onset of anomalous preservation of gas hydrates, Phys. Chem. Chem. Phys. 6, 4917.

Kuhs, W. F., and M.S. Lehmann, 1986, "The structure of ice Ih," Water Science Reviews 2, 1.

Kulikov, M. Yu., A. M. Feigin, S. K. Ignatov, P. G. Sennikov, Th. Bluszcz, and O. Schrems, 2010, "Technical note: VUV photodesorption rates from water ice in the 120-150 K temperature range_significance for noctilucent clouds," Atmos. Chem. Phys. Discuss. 10, 22653.

Kuo, J.L., and M.L. Klein, 2004, "Structure of ice VII and VIII: A quantum mechanical study," J. Phys. Chem. B 108, 19634.

Kuo, J. L., M. L. Klein, and W. F. Kuhs, 2005, "The effect of proton disorder on the structure of ice Ih: A theoretical study," J. Chem. Phys. 123, 134505.

Kuo, J.L., and W.F. Kuhs, 2006, "A first principles study on the structure of ice VI: Static distortion, molecular geometry and proton ordering," J. Phys. Chem. B 110, 3697.

Kuo, J. L., and S. J. Singer, 2003, "Graph invariants for periodic systems: Towards predicting physical properties from the hydrogen bond topology of ice," Phys. Rev. E 67, 016114.

Kuo, J. L, J. V. Coe, S. J. Singer, Y. B. Band, and L. Ojamäe, 2001, "On the use of graph invariants for efficiently generating hydrogen bond topologies and predicting physical properties of water clusters and ice," J. Chem. Phys. 114, 2527.

Kurdi, L., and J. Ponche, 1989, "Theoretical studies of sulphuric acid monohydrate: Neutral or ionic complex?," Chem. Phys. Lett. 158, 111.

Kusaka, I., Z.G. Wang, and J. Seinfeld, 1996, "Monte Carlo simulation of homogeneous binary nucleation: Toward a theory of sulfuric acid-water system," in Nucleation and Atmospheric Aerosols. Proceedings of the 14th International Conference (Pergamon, Oxford).

Kwok, R., 2006, "Contrasts in sea ice deformation and production in the Arctic seasonal and perennial ice zones," J. Geophys. Res. 111, C11S22.

Laaksonen, A., V. Talanquer, and D. W. Oxtoby, 1995, "Nucleation: Measurements, theory, and atmospheric applications," Annu. Rev. Phys. Chem. 46, 489.
Lacy, J. H., J. S. Carr, N. J. II Evans, F. Baas, J. M. Achtermann, and J.F. Arens, 1991, "Discovery of interstellar methane: Observations of gaseous and solid $\mathrm{CH}_{4}$ absorption toward young stars in molecular clouds," Astrophys. J. 376, 556.

Lacy, J. H., H. Faraji, S. A. Sandford, and L. J. Allamandola, 1998, "Unraveling the 10 micron "silicate" feature of protostars: The detection of frozen interstellar ammonia," Astrophys. J. Lett. 501, L105.

Lakhtakia, A., and R. Messier, 2005, Sculptured Thin Films: Nanoengineered Morphology and Optics (SPIE Press, Bellingham, WA).

Latimer, E. R., F. Islam, and S.D. Price, 2008, "Studies of HD formed in excited vibrational states from atomic recombination on cold graphite surfaces," Chem. Phys. Lett. 455, 174.

Lawson, R. P., B. A. Baker, C. G. Schmitt, and T. L. Jensen, 2001, "An overview of microphysical properties of Arctic clouds observed in May and July 1998 during FIRE ACE," J. Geophys. Res. Atmos. 106, 14989.

Lawson, W., 2007, "Environmental conditions, ice facies and glacier behaviour," Glacier Science and Environmental Change (Blackwell Publishing, Malden, MA), 1st ed., p. 319.

Lebensohn, R. A., M. Montagnat, P. Mansuy, P. Duval, J. Meysonnier, and A. Philip, 2009, "Modeling viscoplastic behavior and heterogeneous intracrystalline deformation of columnar ice polycrystals," Acta Mater. 57, 1405.

Lee, S.-H., et al., 2004, "New particle formation observed in the tropical/subtropical cirrus clouds," J. Geophys. Res. 109, D20209.

Legagneux, L., and F. Domine, 2005, "A mean field model of the decrease of the specific surface area of dry snow during isothermal metamorphism," J. Geophys. Res. 110, F04011.

Lejonthun, L. S. E. R., E. A. Svensson, P. U. Andersson, and J. B. C. Pettersson, 2009, "Formation of adsorbed layers by deposition of dinitrogen pentoxide, nitric acid, and water on graphite," J. Phys. Chem. C 113, 7728.

Lellouch, E., J. Crovisier, T. Lim, D. Bockelee-Morvan, K. Leech, M. S. Hanner, B. Altieri, B. Schmitt, F. Trotta, and H. U. Keller, 1998, "Evidence for water ice and estimate of dust production rate in comet Hale-Bopp at 2.9 AU from the Sun," Astron. Astrophys. 339, L9.

Lemaire, J. L., G. Vidali, S. Baouche, M. Chehrouri, H. Chaabouni, and H. Mokrane, 2010, "Competing mechanisms of molecular hydrogen formation in conditions relevant to the interstellar medium," Astrophys. J. Lett. 725, L156.

Lemieux-Dudon, B., E. Blayo, J.-R. Petit, C. Waelbroeck, A. Svensson, C. Ritz, J.-M. Barnola, B. Narcisi, and F. Parrenin, 2010, "Consistent dating for Antarctic and Greenland ice cores," Quaternary Science Reviews 29, 8.

Leppäranta, M., 2004, The Drift of Sea Ice (Springer-Praxis, Heidelberg, Germany).

Leppäranta, M., M. Lensu, P. Kosloff, and B. Veitch, 1995, “The life story of a first-year sea ice ridge," Cold Reg. Sci. Technol. 23, 279.

Leu, M. T., and L.F. Keyser, 2009, "Vapor-deposited water and nitric acid ices: Physical and chemical properties," Int. Rev. Phys. Chem. 28, 53.

Li, Y., and G.A. Somorjai, 2007, "Surface premelting of ice," J. Phys. Chem. C 111, 9631.

Libbrecht, K. G., 2005, “The physics of snow crystals,” Rep. Prog. Phys. 68, 855.

Licandro, J., H. Campins, M. Kelley, K. Hargrove, N. PinillaAlonso, D. Cruikshank, A. S. Rivkin, and J. Emery, 2011, “(65) Cybele: detection of small silicate grains, water-ice, and organics," Astron. Astrophys. 525, A34. 
Liu, R.H., and L.E. Orgel, 1997, "Efficient oligomerization of negatively-charged $\beta$-amino acids at $20^{\circ} \mathrm{C}$," J. Am. Chem. Soc. 119, 4791.

Lliboutry, L., 1954, "The origin of penitentes," J. Glaciol. 2, 331.

Lobban, C., 1998, “Neutron Diffraction Studies of Ices, Ph.D. thesis (University of London).

Lobban, C., J. L. Finney, and W. F. Kuhs, 2000, "The structure and ordering of ices III and V," J. Chem. Phys. 112, 7169.

Loerting, T., D. T. Bowron, and J. L Finney, 2010 (unpublished).

Loerting, T., and N. Giovambattista, 2006, "Amorphous ices: experiments and numerical simulations," J. Phys. Condens. Matter 18, R919.

Loerting, T., C. Salzmann, I. Kohl, E. Mayer, and A. Hallbrucker, 2001, "A second distinct structural "state" of high-density amorphous ice at $77 \mathrm{~K}$ and 1 bar," Phys. Chem. Chem. Phys. 3, 5355.

Loerting, T., K. Winkel, M. Seidl, M. Bauer, C. Mitterdorfer, P. H. Handle, E. Mayer, J. L. Finney, and D. T. Bowron, 2011, "How many amorphous ices are there?," Phys. Chem. Chem. Phys. (to be published).

Löfgren, P., P. Ahlström, D. V. Chakarov, J. Lausmaa, and B. Kasemo, 1996, "Substrate dependent sublimation kinetics of mesoscopic ice films," Surf. Sci. Lett. 367, L19.

Lohmann, U., 2002, "A glaciation indirect aerosol effect caused by soot aerosols," Geophys. Res. Lett. 29, 1052.

Lohmann, U., and J. Feichter, 2005, "Global indirect aerosol effects: a review," Atmos. Chem. Phys. 5, 715.

Loulergue, L., F. Parrenin, T. Blunier, J.-M. Barnola, R. Spahni, A. Schilt, G. Raisbeck, and J. Chappellaz, 2007, "New constraints on the gas age-ice age difference along the EPICA ice cores, 0-50 kyr," Clim. Past 3, 527.

Lowe, D., and A. R. MacKenzie, 2008, "Polar stratospheric cloud microphysics and chemistry," J. Atmos. Sol. Terr. Phys. 70, 13.

Lund Myhre, C., H. Grothe, A. Gola, and C. Nielsen, 2005, "Optical constants of $\mathrm{HNO}_{3} / \mathrm{H}_{2} \mathrm{O}$ and $\mathrm{H}_{2} \mathrm{SO}_{4} / \mathrm{HNO}_{3} / \mathrm{H}_{2} \mathrm{O}$ at low temperatures in the infrared region," J. Phys. Chem. A 109, 7166.

Luo, J., and Y.-M. Chiang, 2008, "Wetting and prewetting on ceramic surfaces," Annu. Rev. Mater. Res. 38, 227.

Lynch, D. K., K. Sassen, D. O'C. Starr, and G. Stephens, 2002, Cirrus (Oxford University Press, New York).

Mader, H. M., 1992, "Observations of the water-vein system in polycrystalline ice,” J. Glaciol. 38, 333.

Magee, N., A. M. Moyle, and D. Lamb, 2006, "Experimental determination of the deposition coefficient of small cirrus-like ice crystals near $-50{ }^{\circ}$ C," Geophys. Res. Lett. 33, L17 813 .

Malyk, S., G. Kumi, H. Reisler, and C. Wittig, 2007, "Trapping and release of $\mathrm{CO}_{2}$ guest molecules by amorphous ice," J. Phys. Chem. A 111, 13365.

Manca, C., C. Martin, A. Allouche, and P. Roubin, 2001, "Experimental and theoretical reinvestigation of $\mathrm{CO}$ adsorption on amorphous ice," J. Phys. Chem. B 105, 12861.

Mantz, Y. A., F. M. Geiger, L. T. Molina, M. J. Molina, and B. L. Trout, 2002, "A theoretical study of the interaction of $\mathrm{HCl}$ with crystalline NAT," J. Phys. Chem. A 106, 6972.

Marecal, V., M. Pirre, E. D. Riviere, N. Pouvesle, J. N. Crowley, S.R. Freitas, and K.M. Longo, 2010, "Modelling the reversible uptake of chemical species in the gas phase by ice particles formed in a convective cloud," Atmos. Chem. Phys. 10, 4977.

Margesin, R., 2008, Ed., Permafrost Soils (Springer, New York).

Marsan, D., J. Weiss, J. P. Métaxian, J. Grangeon, P.-F. Roux, and J. Haapala, 2011, "Low frequency bursts of horizontally-polarized waves in the arctic sea-ice cover," J. Glaciol. 57, 231.
Marshall, S.J., 2007, "Modelling glacier response to climate change," in Glacier Science and Environmental Change, edited by P.G. Knight (Blackwell Publishing, Malden, MA), 1st ed., p. 163.

Martin, S. T., 2000, "Phase transitions of aqueous atmospheric particles," Chem. Rev. 100, 3403.

Maslanik, J. A., C. Fowler, J. Stroeve, S. Drobot, J. Zwally, D. Yi, and W. Emery, 2007, "A younger, thinner ice cover: Increased potential for rapid, extensive sea-ice loss," Geophys. Res. Lett. 34, L24 501.

Mastrapa, R. M., S. A. Sandford, T. L. Roush, D. P. Cruikshank, and C. M. Dalle Ore, 2009, "Optical constants of amorphous and crystalline $\mathrm{H}_{2} \mathrm{O}$-ice: $2.5-22 \mu \mathrm{m}\left(4000-455 \mathrm{~cm}^{-1}\right)$ optical constants of $\mathrm{H}_{2} \mathrm{O}$-ice," Astrophys. J. 701, 1347.

Maté, B., O. Gálvez, V.J. Herrero, and R. Escribano, 2009, "Infrared spectra and thermodynamic properties of $\mathrm{CO}_{2}$ /methanol ices," Astrophys. J. 690, 486.

Matsuo, T., Y. Tajima, and H. Suga, 1986, "Calorimetric study of a phase transition in $\mathrm{D}_{2} \mathrm{O}$ ice Ih doped with KOD: ice XI," J. Phys. Chem. Solids 47, 165.

Matykiewiczova, N., J. Klanova, and P. Klan, 2007, "Photochemical degradation of PCBs in snow," Environ. Sci. Technol. 41, 8308.

Maxwell, J.C., 1871, "To the chief musician upon Nabla: A Tyndallic ode," Nature (London) 4, 291.

Mayer, E., 1985, "New method for vitrifying water and other liquids by rapid cooling of their aerosols," J. Appl. Phys. 58, 663.

Mayer, E., and P. Brüggeller, 1982, "Vitrification of pure liquid water by high pressure jet freezing," Nature (London) 298, 715.

Mayer, E., and R. Pletzer, 1986, "Astrophysical implications of amorphous ice-a microporous solid," Nature (London) 319 , 298.

Mazzega, E., U. del Pennino, A. Loria, and S. Mantovani, 1976, "Volta effect and liquidlike layer at the ice surface," J. Chem. Phys. 64, 1028.

McCleese, D. J., et al., 2010, "Structure and dynamics of the Martian lower and middle atmosphere as observed by the Mars Climate Sounder: Seasonal variations in zonal mean temperature, dust, and water ice aerosols," J. Geophys. Res. 115, 12016.

McNeill, V. F., F. M. Geiger, T. Loerting, B. L. Trout, L. T. Molina, and M. Molina, 2007, "Interaction of hydrogen chloride with ice surfaces: The effect of grain size, surface roughness, and surface disorder," J. Phys. Chem. A 111, 6274.

McNeill, V.F., T. Loerting, F. M. Geiger, B. L. Trout, and M. J. Molina, 2006, "Hydrogen chloride-induced surface disordering on ice," Proc. Natl. Acad. Sci. U.S.A. 103, 9422.

Meierhenrich, U. J., 2008, Amino Acids and the Asymmetry of Life (Springer-Verlag, Berlin/Heidelberg).

Meierhenrich, U.J., G. M.M. Caro, J.H. Bredehöft, and E.K. Jessberger, 2004, "Identification of diamino acids in the Murchison meteorite," Proc. Natl. Acad. Sci. U.S.A. 101, 9182.

Mellenthin, J., A. Karma, and M. Plapp, 2008, "Phase-field crystal study of grain-boundary premelting," Phys. Rev. B 78, 184110.

Mellor, M.M., 1986, "Mechanical behavior of sea ice," in Geophysics of Sea Ice, edited by N. Untersteiner, NATO Advanced Science Institutes Ser. B, Physics, Vol. 146 (Plenium Press, New York), p. 165.

Messier, R., V.C. Venugopal, and P.D. Sunal, 2000, "Origin and evolution of sculptured thin films," J. Vac. Sci. Technol. A 18, 1538.

Meyers, M. P., P. J. Demott, and W. R. Cotton, 1992, "New primary ice-nucleation parameterizations in an explicit cloud model," J. Appl. Meteorol. 31, 708. 
Minčeva-Šukarova, B., G. Slark, and W.F. Sherman, 1988, "The Raman spectra of the KOH-doped ice polymorphs: V and VI," J. Mol. Struct. 175, 289.

Mishchenko, M. I., W. B. Rossow, A. Macke, and A. A. Lacis, 1996, "Sensitivity of cirrus cloud albedo, bidirectional reflectance and optical thickness retrieval accuracy to ice particle shape," J. Geophys. Res. 101, 16973.

Mishima, O., L. D. Calvert, and E. Whalley, 1984, “"Melting ice' I at $77 \mathrm{~K}$ and 10 kbar: a new method of making amorphous solids," Nature (London) 310, 393.

Mishima, O., L. D. Calvert, and E. Whalley, 1985, “An apparently first-order transition between two amorphous phases of ice induced by pressure," Nature (London) 314, 76.

Mishima, O., and H. E. Stanley, 1998, "The relationship between liquid, supercooled and glassy water," Nature (London) 396, 329.

Mishima, O., and Y. Suzuki, 2002, "Propagation of the polyamorphic transition of ice and the liquid-liquid critical point," Nature (London) 419, 599.

Mitlin, S., and K. T. Leung, 2002, "Film growth of ice by vapor deposition at 128-185 K studied by Fourier transform infrared reflection-absorption spectroscopy: Evolution of the $\mathrm{OH}$ stretch and the dangling bond with film thickness," J. Phys. Chem. B 106, 6234.

Möhler, O., S. Benz, H. Saathoff, M. Schnaiter, R. Wagner, J. Schneider, S. Walter, V. Ebert, and S. Wagner, 2008, "The effect of organic coating on the heterogeneous ice nucleation efficiency of mineral dust aerosols," Environ. Res. Lett. 3, 025007.

Möhler, O., H. Bunz, and O. Stetzer, 2006, "Homogeneous nucleation rates of nitric acid dihydrate (NAD) at simulated stratospheric conditions-Part II: Modelling," Atmos. Chem. Phys. Discuss. 6, 2119.

Möhler, O., P.J. DeMott, G. Vali, and Z. Levin, 2007, "Microbiology and atmospheric processes: the role of biological particles in cloud physics," Biogeosciences 4, 1059.

Möhler, O., et al., 2006, "Efficiency of the deposition mode ice nucleation on mineral dust particles," Atmos. Chem. Phys. 6, 3007.

Möhler, O., C. Linke, H. Saathoff, M. Schnaiter, R. Wagner, A. Mangold, M. Krämer, and U. Schurath, 2005, "Ice nucleation on flame soot aerosol of different organic carbon content," Meteorol. Z. 14, 477.

Mokrane, H., H. Chaabouni, M. Accolla, E. Congiu, F. Dulieu, M. Chehrouri, and J. L. Lemaire, 2009, "Experimental evidence for water formation via ozone hydrogenation on dust grains at $10 \mathrm{~K}$," Astrophys. J. Lett. 705, L195.

Molina, M. J., T.L. Tso, L. T. Molina, and F. C. Y. Wang, 1987, "Antarctic stratospheric chemistry of chlorine nitrate, hydrogen chloride, and ice: Release of active chlorine," Science 238, 1253.

Monnard, P., A. Kanavarioti, and D. W. Deamer, 2003, "Eutectic phase polymerization of activated ribonucleotide mixtures yields quasi-equimolar incorporation of purine and pyrimidine nucleobases," J. Am. Chem. Soc. 125, 13734.

Montmessin, F., 2012, Habilitation thesis, "Observation and modeling of terrestrial-type atmospheres" (Université St. Quentin en Yvelines).

Moore, E. B., E. de la Llave, K. Welke, D. A. Scherlis, and V. Molinero, 2010, "Freezing, melting and structure of ice in a hydrophilic nanopore," Phys. Chem. Chem. Phys. 12, 4124.

Moore, E. B., and V. Molinero, 2011, "Is it cubic? Ice crystallization from deeply supercooled water," Phys. Chem. Chem. Phys. 13, 20008.

Moore, M. H., R. F. Ferrante, R. L. Hudson, J. A. Nuth III, and B. Donn, 1994, "Infrared spectra of crystalline phase ices condensed on silicate smokes at $T<20 \mathrm{~K}$," Astrophys. J. 428, L81.
Moore, M. H., and R. L. Hudson, 2000, "IR detection of $\mathrm{H}_{2} \mathrm{O}_{2}$ at $80 \mathrm{~K}$ in ion-irradiated laboratory ices relevant to Europa," Icarus 145, 282.

Morishige, K., and H. Uematsu, 2005, "The proper structure of cubic ice confined in mesopores," J. Chem. Phys. 122, 044711.

Morrison, D., T. V. Johnson, T. M. Shoemaker, L. A. Soderblom, J. Thomas, P. Veverka, and B. A. Smith, 1984, "Satellites of Saturn: Geological Perspective," in Saturn (University of Arizona Press, Tucson, AZ).

Moulton, V., P. P. Gardner, R. F. Pointon, L. K. Creamer, G. B. Jameson, and D. Penny, 2000, "RNA folding argues against a hot-start origin of life," J. Mol. Evol. 51, 416 [http://www .springerlink.com/content/08tu0ra5kx9cuhbw/].

Mousis, O., and Y. Alibert, 2006, "Modeling the Jovian subnebula. II. Composition of regular satellite ices," Astron. Astrophys. 448, 771.

Mulvaney, R., E. Wolff, and K. Oates, 1988, "Sulphuric acid at grain boundaries in Antarctic ice," Nature (London) 331, 247.

Mundy, C. J., and I-F. W. Kuo, 2006, "First-principles approaches to the structure and reactivity of atmospherically relevant aqueous interfaces," Chem. Rev. 106, 1282.

Muñoz Caro, G. M., G. Matrajt, E. Dartois, M. Nuevo, L. d'Hendecourt, D. Deboffle, G. Montagnac, N. Chauvin, C. Boukari, and D. Le Du, 2006, "Nature and evolution of the dominant carbonaceous matter in interplanetary dust particles: effects of irradiation and identification with a type of amorphous carbon," Astron. Astrophys. 459, 147.

Muñoz Caro, G. M., U. J. Meierhenrich, W. A. Schutte, B. Barbier, A. Arcones Segovia, H. Rosenbauer, W. H.-P. Thiemann, A. Brack, and J. M. Greenberg, 2002, "Amino acids from ultraviolet irradiation of interstellar ice analogues," Nature (London) 416, 403.

Murphy, D. M., and T. Koop, 2005, "Review of the vapour pressures of ice and supercooled water for atmospheric applications," Q. J. R. Meteorol. Soc. 131, 1539.

Murray, B. J., and A.K. Bertram, 2007, "Strong dependence of cubic ice formation on droplet ammonium to sulfate ratio," Geophys. Res. Lett. 34, L16810.

Murray, B.J., and A.K. Bertram, 2008, "Inhibition of solute crystallisation in aqueous $\mathrm{H}^{+}-\mathrm{NH}_{4}^{+}-\mathrm{SO}_{4}^{2-}-\mathrm{H}_{2} \mathrm{O}$ droplets," Phys. Chem. Chem. Phys. 10, 3287.

Murray, B. J., and E. J. Jensen, 2010, "Homogeneous nucleation of amorphous solid water particles in the upper mesosphere," J. Atmos. Sol. Terr. Phys. 72, 51.

Murray, B.J., D. A. Knopf, and A.K. Bertram, 2005, "The formation of cubic ice under conditions relevant to earth's atmosphere," Nature (London) 434, 202.

Murray, B. J., and J. M. C. Plane, 2005, "Modelling the impact of noctilucent cloud formation on atomic oxygen and other minor constituents of the summer mesosphere," Atmos. Chem. Phys. 5, 1027.

Murray, B.J., et al., 2010, "Heterogeneous nucleation of ice particles on glassy aerosols under cirrus conditions," Nature Geosci. 3, 233.

Någård, M.B., et al., 2002, "Dissociative recombination of $\mathrm{D}^{+}\left(\mathrm{D}_{2} \mathrm{O}\right)_{2}$ water cluster ions with free electrons," J. Chem. Phys. 117, 5264.

Nakaya, U., 1954, Snow Crystals: Natural and Artificial (Harvard University Press, Cambridge).

Narten, A. H., C. G. Venkatesh, and S. A. Rice, 1976, "Diffraction pattern and structure of amorphous solid water at 10 and $77^{\circ} \mathrm{K}$," J. Chem. Phys. 64, 1106.

Nash, D. B., and B. H. Betts, 1995, "Ices on Io-Composition and Texture," in Solar System Ices (Kluwer, Dordrecht), Vol. 227. 
Nelmes, R. J., J. S. Loveday, T. Strässle, M. Guthrie, C. L. Bull, G. Hamel, and S. Klotz, 2006, "Annealed high-density amorphous ice under pressure," Nature Phys. 2, 414.

Neufeld, J. A., R. E. Goldstein, and M. G. Worster, 2010, "On the mechanisms of icicle evolution," J. Fluid Mech. 647, 287.

Niemann, H.B., S. K. Atreya, J.E. Demick, D. Gautier, J. A. Haberman, D. N. Harpold, W. T. Kasprzak, J. I. Lunine, T. C. Owen, and F. Raulin, 2010, "Composition of Titan's lower atmosphere and simple surface volatiles as measured by the Cassini-Huygens probe gas chromatograph mass spectrometer experiment," J. Geophys. Res. 115, 12006.

Noel, V., E. Chepfer, M. Haeffelin, and Y. Morille, 2006, "Classification of ice crystal shapes in midlatitude ice clouds from three years of lidar observations over the SIRTA observatory," J. Atmos. Sci. 63, 2978.

Noel, V., H. Chepfer, G. Ledanois, A. Delaval, and P. H. Flamant, 2002, "Classification of particle effective shape ratios in cirrus clouds based on the lidar depolarization ratio," Appl. Opt. 41, 4245.

Noel, V., D. M. Winker, M. McGill, and P. Lawson, 2004, "Classification of particle shapes from lidar depolarization ratio in convective ice clouds compared to in situ observations during CRYSTAL-FACE," J. Geophys. Res. Atmos. 109.

Notesco, G., and A. Bar-Nun, 1996, "Enrichment of CO over $\mathrm{N}_{2}$ by their trapping in amorphous ice and implications to comet $\mathrm{P} /$ Halley," Icarus 122, 118.

Notesco, G., and A. Bar-Nun, 2000, "The effect of methanol clathrate-hydrate formation and other gas-trapping mechanisms on the structure and dynamics of cometary ices," Icarus 148, 456.

Nye, J. F, 1992, "Water veins and lenses in polycrystalline ice," in Physics and Chemistry of Ice (Hokkaido University Press, Sapporo), p. 200.

Oba, Y., N. Miyauchi, H. Hidaka, T. Chigai, N. Watanabe, and A. Kouchi, 2009, "Formation of compact amorphous $\mathrm{H}_{2} \mathrm{O}$ ice by codeposition of hydrogen atoms with oxygen molecules on grain surfaces," Astrophys. J. 701, 464.

Obbard, R., I. Baker, and K. Sieg, 2006, "Using electron backscatter diffraction patterns to examine recrystallization in polar ice sheets," J. Glaciol. 52, 546.

Öberg, K. I., H. J. Fraser, A. C. A. Boogert, S. E. Bisschop, G. W. Fuchs, E. F. van Dishoeck, and H. Linnartz, 2007, "Effects of $\mathrm{CO}_{2}$ on $\mathrm{H}_{2} \mathrm{O}$ band profiles and band strengths in mixed $\mathrm{H}_{2} \mathrm{O}: \mathrm{CO}_{2}$ ices," Astron. Astrophys. 462, 1187.

Öberg, K. I., E. F. van Dishoeck, H. Linnartz, and S. Andersson, 2010, "The effect of $\mathrm{H}_{2} \mathrm{O}$ on ice photochemistry," Astrophys. J. 718, 832.

Oguro, M., and T. Hondoh, 1988, "Stacking faults in ice crystals," in Lattice Defects in Ice Crystals, edited by A. Higashi (Hokkaido University Press, Sapporo), p. 49.

Ohmura, A., M. Wild, and L. Bengtsson, 1996, "A possible change in mass balance of Greenland and Antarctic ice sheets in the coming century," J. Clim. 9, 2124.

Oikkonen, A., and J. Haapala, 2011, "Variability and changes of Arctic sea ice draft distribution-submarine sonar measurements revisited," Cryosphere 5, 917.

Öjekull, J., et al., 2007, "Dissociative recombination of $\mathrm{H}^{+}\left(\mathrm{H}_{2} \mathrm{O}\right)_{3}$ and $\mathrm{D}^{+}\left(\mathrm{D}_{2} \mathrm{O}\right)_{3}$ water cluster ions with electrons: Cross sections and branching ratios," J. Chem. Phys. 127, 194301.

Öjekull, J., et al., 2008, "Dissociative recombination of water cluster ions with free electrons: cross sections and branching ratios," J. Chem. Phys. 128, 044311.

O'Keefe, J.A., 1980, “The terminal Eocene event: formation of a ring system around the Earth?," Nature (London) 285, 309 .
Orgel, L.E., 1998, "The origin of life-a review of facts and speculations," Trends Biochem. Sci. 23, 491.

Paige, R.A., 1970, "Stalactite growth beneath sea ice," Science 167, 171 .

Palumbo, M.E., T.R. Geballe, and A.G.G.M. Tielens, 1997, "Solid carbonyl sulfide (OCS) in dense molecular clouds," Astrophys. J. 479, 839.

Park, S.-C., E.-S. Moon, and H. Kang, 2010, "Some fundamental properties and reactions of ice surfaces at low temperatures," Phys. Chem. Chem. Phys. 12, 12000.

Parmeter, R. R., 1975, “A model of simple rafting in sea ice," J. Geophys. Res. 80, 1948.

Pat-El, I., D. Laufer, G. Notesco, and A. Bar-Nun, 2009, "An experimental study of the formation of an ice crust and migration of water vapor in a comet's upper layers," Icarus 201, 406.

Paterson, W. S. B., 1994, The Physics of Glaciers (Reed Elsevier, Oxford), 3rd ed.

Pedersen, C.A., E. Roecker, M. Lüthje, and J.-G. Winter, 2009, "A new sea ice albedo scheme including melt ponds for ECHAM5 general circulation model,” J. Geophys. Res. 114, D08101.

Perets, H. B., O. Biham, G. Manico, V. Pirronello, J. Roser, S. Swords, and G. Vidali, 2005, "Molecular hydrogen formation on ice under interstellar conditions," Astrophys. J. 627, 850.

Perovich, D. K., and J. A. Richter-Menge, 1994, "Surface characteristics of lead ice," J. Geophys. Res. 99, 16341.

Pertaya, N., Y. Celik, C. L. DiPrinzio, J. S. Wettlaufer, P. L. Davies, and I. Braslavsky, 2007, "Growth-melt asymmetry in ice crystals under the influence of spruce budworm antifreeze protein," J. Phys. Condens. Matter 19, 412101.

Pertaya, N., C. B. Marshall, C. L. DiPrinzio, L. A. Wilen, E. S. Thomson, J. S. Wettlaufer, P. L. Davies, and I. Braslavsky, 2007, "Fluorescence microscopy evidence for quasi-permanent attachment of antifreeze proteins to ice surfaces," Biophys. J. 92, 3663.

Peter, T., C. Marcolli, P. Spaichinger, T. Corti, M. C. Baker, and T. Koop, 2006, "When dry air is too humid," Science 314, 1399.

Petrenko, V. F., and R. W. Whitworth, 1999, Physics of Ice (Oxford University Press, Oxford).

Pinzer, B., M. Kerbrat, T. Huthwelker, H.W. Gäggeler, M. Schneebeli, and M. Ammann, 2010, "Diffusion of $\mathrm{NO}_{x}$ and HONO in snow: A laboratory study," J. Geophys. Res. 115, D03304.

Pinzer, B., and M. Schneebeli, 2009, "Snow metamorphism under alternating temperature gradients: Morphology and recrystallization in surface snow," Geophys. Res. Lett. 36, L23503.

Pitter, R. L., and H. R. Pruppacher, 1973, "Wind-tunnel investigation of freezing of small water drops falling at terminal velocity in air,” Q. J. R. Meteorol. Soc. 99, 540.

Poole, P.H., F. Sciortino, U. Essman, and H.E. Stanley, 1992, "Phase behaviour of metastable water," Nature (London) 360, 324.

Pöschl, U., et al., 2010, "Rainforest Aerosols as Biogenic Nuclei of Clouds and Precipitation in the Amazon," Science 329, 1513.

Post, A., and E. R. LaChapelle, 1971, Glacier Ice (University of Washington Press, Seattle, WA).

Pratt, K. A., P. J. DeMott, J. R. French, Z. Wang, D. L. Westphal, A. J. Heymsfield, C. H. Twohy, A. J. Prenni, and K. A. Prather, 2009, "In situ detection of biological particles in cloud icecrystals," Nature Geosci. 2, 398.

Pratte, P., H. van den Bergh, and M. J. Rossi, 2006, "The kinetics of $\mathrm{H}_{2} \mathrm{O}$ vapor condensation and evaporation on different 
types of ice in the range 130-210 K," J. Phys. Chem. A 110, 3042.

Prialnik, D., J. Benkhoff, and M. Podolak, 2003, "Modeling the structure and activity of comet nuclei," in Comets II, edited by M. C. Festou, H. U. Keller, and H. A. Weaver (University of Arizona Press, Tucson, AZ), p. 359.

Price, P. B., 2007, "Microbial life in glacial ice and implications for a cold origin of life," FEMS Microbiol. Ecol. 59, 217.

Price, P. B., 2010, "Microbial life in martian ice: A biotic origin of methane on Mars?," Planet. Space Sci. 58, 1199.

Pritchard, H., R. Arthern, D. Vaughan, and L. Edwards, 2009, "Extensive dynamic thinning on the margins of the Greenland and Antarctic ice sheets," Nature (London) 461, 971.

Pruppacher, H. R., and J. D. Klett, 1997, Microphysics of Clouds and Precipitation (Reidel, Dordrecht, Holland).

Pummer, B. G., H. Bauer, J. Bernardi, S. Bleicher, and H. Grothe, 2012, "Suspendable macromolecules are responsible for ice nucleation activity of birch and conifer pollen ," Atmos. Chem. Phys. 12, 2541.

Rampal, P., J. Weiss, and D. Marsan, 2009, "Positive trend in the mean speed and deformation rate of Arctic sea ice," J. Geophys. Res. 114, C05013.

Rapp, M., and G.E. Thomas, 2006, "Modeling the microphysics of mesospheric ice particles: Assessment of current capabilities and basic sensitivities," J. Atmos. Sol. Terr. Phys. 68, 715.

Raviv, U., P. Laurat, and J. Klein, 2001, "Fluidity of water confined to subnanometre films," Nature (London) 413, 51.

Reitz, P., et al., 2011, "Surface modification of mineral dust particles by sulphuric acid processing: implications for ice nucleation abilities," Atmos. Chem. Phys. 11, 7839.

Rempel, A., E. Waddington, J. Wettlaufer, and M. Worster, 2001, "Possible displacement of the climate signal in ancient ice by premelting and anomalous diffusion," Nature (London) 411, 568.

Richardson, H. H., P. J. Wooldridge, and J.P. Devlin, 1985, “FT-IR spectra of vacuum deposited clathrate hydrate of oxirane $\mathrm{H}_{2} \mathrm{~S}$, THF, and ethane," J. Chem. Phys. 83, 4387.

Rignot, E., and P. Kanagaratnam, 2006, "Changes in the velocity structure of the Greenland ice sheet," Science 311, 986.

Rigor, I. G., and J. M. Wallace, 2004, "Variations in the age of the Arctic sea-ice and summer sea-ice extent," Geophys. Res. Lett. 31, L09401.

Rigor, I. G., J. M. Wallace, and R. L. Colony, 2002, "Response of sea ice to the Arctic Oscillation," J. Clim. 15, 2648.

Riikonen, M., M. Sillanpää, L. Virta, D. Sullivan, J. Moilanen, and I. Luukkonen, 2000, "Halo observations provide evidence of airborne cubic ice in the earths atmosphere," Appl. Opt. 39, 6080.

Rivkin, A. S., and J. P. Emery, 2010, "Detection of ice and organics on an asteroidal surface," Nature (London) 464, 1322.

Rode, B.M., 1999, "Peptides and the origin of life," Peptides 20, 773.

Roser, J.E., G. Manicò, V. Pirronello, and G. Vidali, 2002, "Formation of molecular hydrogen on amorphous water ice: Influence of morphology and ultraviolet exposure," Astrophys. J. 581, 276.

Rothrock, D. A., Y. Yu, and G. A. Maykut, 1999, "Thinning of the Arctic sea-ice cover," Geophys. Res. Lett. 26, 3469.

Rothrock, D. A., and J. Zhang, 2005, "Arctic ocean sea ice volume: What explains its recent depletion?," J. Geophys. Res. 110, C01002.

Rozenberg, M., and A. Loewenschuss, 2009, "Matrix isolation infrared spectrum of the sulfuric acid-monohydrate complex:
New assignments and resolution of the "missing H-bonded $\nu$ (oh) band" issue," J. Phys. Chem. A 113, 4963.

Russell, III, J.M., et al., 2009, "The aeronomy of ice in the mesosphere (AIM) mission: Overview and early science results," J. Atmos. Sol. Terr. Phys. 71, 289.

Sadtchenko, V., M. Brindza, M. Chonde, B. Palmore, and R. Eom, 2004, "The vaporization rate of ice at temperatures near its melting point," J. Chem. Phys. 121, 11980.

Sadtchenko, V., and G.E. Ewing, 2002, "Interfacial melting of thin ice films: An infrared study," J. Chem. Phys. 116, 4686.

Safarik, D. J., R. J. Meyer, and C. B. Mullins, 2003, “Thickness dependent crystallization kinetics of sub-micron amorphous solid water films," J. Chem. Phys. 118, 4660.

Safarik, D. J., and C.B. Mullins, 2004, "The nucleation rate of crystalline ice in amorphous solid water," J. Chem. Phys. 121, 6003.

Salzmann, C., P. G. Radaelli, A. Hallbrucker, E. Mayer, and J. L. Finney, 2006, "The preparation and structures of hydrogen ordered phases of ice," Science 311, 1758.

Salzmann, C., P. G. Radaelli, E. Mayer, and J. L. Finney, 2009, “Ice XV: A new thermodynamically stable phase of ice," Phys. Rev. Lett. 103, 105701.

Salzmann, C. G., T. Loerting, I. Kohl, E. Mayer, and A. Hallbrucker, 2002, "Pure ice IV from high-density amorphous ice," J. Phys. Chem. B 106, 5587.

Sandford, S. A., and L. J. Allamandola, 1990, "The volume- and surface-binding energies of ice systems containing $\mathrm{CO}, \mathrm{CO}_{2}$ and $\mathrm{H}_{2} \mathrm{O}$," Icarus 87, 188.

Saunders, C., 2008, "Charge separation mechanisms in clouds," Space Sci. Rev. 137, 335.

Saunders, R. W., et al., 2010, "An aerosol chamber investigation of the heterogeneous ice nucleating potential of refractory nanoparticles," Atmos. Chem. Phys. 10, 1227.

Sazaki, G., S. Zepeda, S. Nakatsubo, E. Yokoyama, and Y. Furukawa, 2010, "Elementary steps at the surface of ice crystals visualized by advanced optical microscopy," Proc. Natl. Acad. Sci. U.S.A. 107, 19702.

Scheuer, E., J.E. Dibb, C. Twohy, D. C. Rogers, A. J. Heymsfield, and A. Bansemer, 2010, "Evidence of nitric acid uptake in warm cirrus anvil clouds during the NASA TC4 campaign," J. Geophys. Res. Atmos. 115, D00J03.

Schick, M., 1990, "Introduction to wetting phenomena," in Liquids at Interfaces, Les Houches Lectures 1988, edited by J. Charvolin, J. F. Joanny, and J. Zinn-Justin (Elsevier, New York), Vol. XLVIII, Chap. 9.

Schick, M., and W.-H. Shih, 1987, "Z(n) model of grain-boundary wetting," Phys. Rev. B 35, 5030.

Schiermeier, Q., 2001, "Fears grow over melting permafrost," Nature (London) 409, 751.

Schilling, G., 2011, "Dark ice on a hot planet," Science 334, 162 [http://www.sciencemag.org/content/334/6053].

Schmitt, B., R. Grim, and M. Greenberg, 1989, "Spectroscopy and physico-chemistry of $\mathrm{CO}: \mathrm{H}_{2} \mathrm{O}$ and $\mathrm{CO}_{2}: \mathrm{H}_{2} \mathrm{O}$ ices," Infrared Spectroscopy in Astronomy (ESA, Noordwijk, The Netherlands), p. 213.

Schopf, J. W., 2002, Life's Origin: The Beginnings of Biological Evolution (University of California Press, Berkeley/Los Angeles, CA).

Schulson, E. M., and P. Duval, 2009, Creep and Fracture of Ice (Cambridge University Press, Cambridge, England).

Schulze-Makuch, D., and L. N. Irwin, 2008, Life in the Universe: Expectations and Constraints (Springer, Berlin/Heidelberg), 2nd ed. 
Schutte, W. A., 2002, "The low temperature crystallization effect reevaluated," Astron. Astrophys. 386, 1103.

Schutte, W. A., P. A. Gerakines, T. R. Geballe, E. F. van Dishoeck, and J.M. Greenberg, 1996, "Discovery of solid formaldehyde toward the protostar GL 2136: observations and laboratory simulation," Astron. Astrophys. 309, 633.

Schweizer, J., 2008, "Snow avalanche formation and dynamics," Cold Reg. Sci. Technol. 54, 153.

Schweizer, J., J. B. Jamieson, and M. Schneebeli, 2003, "Snow avalanche formation," Rev. Geophys. 41, 1016.

Schwendinger, M. G., R. Tattler, S. Saetia, K. R. Liedl, R. T. Kroemer, and B. M. Rode, 1995, "Salt induced peptide formation: on the selectivity of the copper induced peptide formation under possible prebiotic conditions," Inorg. Chim. Acta 228, 207.

Shallcross, F. V., and G.B. Carpenter, 1957, "X-ray diffraction study of the cubic phase of ice," J. Chem. Phys. 26, 782.

Shapiro, R., 2000, "A replicator was not involved in the origin of life," IUBMB Life 49, 173.

Shaw, R. A., and D. Lamb, 1999, "Homogeneous freezing of evaporating cloud droplets," Geophys. Res. Lett. 26, 1181.

Shean, D. E., J. W. Head, J. L. Fastook, and D. R. Marchant, 2007, "Recent glaciation at high elevations on Arsia Mons, Mars: Implications for the formation and evolution of large tropical mountain glaciers," J. Geophys. Res. 112, 3004.

Shen, H.H., W. D. Hibler III, and M. Leppäranta, 1986, "On applying granular flow theory to a deforming broken ice field," Acta Mech. 63, 143.

Shilling, J. E., M. A. Tolbert, O. B. Toon, E. J. Jensen, B. J. Murray, and A. K. Bertram, 2006, "Measurements of the vapor pressure of cubic ice and their implications for atmospheric ice clouds," Geophys. Res. Lett. 33, 026671.

Shoji, H., and C. C. Langway, 1982, "Air hydrate inclusions in fresh ice core," Nature (London) 298, 548.

Showalter, M. R., and J. J. Lissauer, 2006, "The second ring-moon system of Uranus: Discovery and dynamics," Science 311, 973.

Sunshine, J. M., et al., 2006, "Exposed Water Ice Deposits on the Surface of Comet 9P/Tempel 1," Science 311, 1453.

Simpson, W., et al., 2007, "Halogens and their role in polar boundary-layer ozone depletion," Atmos. Chem. Phys. 7, 4375 .

Sloan, E. D., and C. A. Koh, 2008, Clathrate Hydrates of Natural Gases (CRC Press, Boca Raton, FL).

Smith, C. S., 1948, "Grains, phases, and interfaces-an interpretation of microstructure," Trans. Am. Inst. Min., Metall. Pet. Eng. 175, 15.

Smith, C.S., 1964, "Structure, substructure and superstructure," Rev. Mod. Phys. 36, 524.

Smith, R.S., C. Huang, E. K. L. Wong, and B.D. Kay, 1996, "Desorption and crystallization kinetics in nanoscale thin films of amorphous water ice," Surf. Sci. 367, L13.

Smith, R. S., C. Huang, E. K. L. Wong, and B. D. Kay, 1997, “The molecular volcano: Abrupt $\mathrm{CCl}_{4}$ desorption driven by the crystallization of amorphous solid water," Phys. Rev. Lett. 79, 909.

Sohl, F., 2010, "Revealing Titans interior," Science 327, 1338.

Sohl, F., T. Spohn, D. Breuer, and K. Nagel, 2002, "Implications from Galileo observations on the interior structure and chemistry of the Galilean satellites," Icarus 157, 104.

Solomon, S., S. Borrmann, R. R. Garcia, R. Portmann, L. Thomason, L. R. Poole, D. Winker, and M.wP. McCormick, 1997, "Heterogeneous chlorine chemistry in the tropopause region,” J. Geophys. Res. Atmos. 102, 21411.

Souda, R., 2003, “Temperature-programmed time of flight secondary ion mass spectrometry study of hydration of ammonia and formic acid adsorbed on the water-ice surface," J. Chem. Phys. 119, 2774.

Staykova, D. K., W. F. Kuhs, A. N. Salamatin, and T. Hansen, 2003, "Formation of porous gas hydrates from ice powders: Diffraction experiments and multistage model," J. Phys. Chem. B 107, 10299.

Steffen, A., et al., 2008, "A synthesis of atmospheric mercury depletion event chemistry in the atmosphere and snow," Atmos. Chem. Phys. 8, 1445.

Stetzer, O., O. Möhler, R. Wagner, S. Benz, H. Saathoff, H. Bunz, and O. Indris, 2006, "Homogeneous nucleation rates of nitric acid dihydrate (NAD) at simulated stratospheric conditions-Part I: Experimental results," Atmos. Chem. Phys. Discuss. 6, 2091.

Stratmann, F., A. Kiselev, S. Wurzler, M. Wendisch, J. Heintzenberg, R. J. Charlson, K. Diehl, H. Wex, and S. Schmidt, 2004, "Laboratory studies and numerical simulations of cloud droplet formation under realistic supersaturation conditions," J. Atmos. Ocean. Technol. 21, 876.

Strazzulla, G., and R.E. Johnson, 1991, "Irradiation effects on comets and cometary debris," Comets in the Post-Halley Era, ASSL Series (Kluwer Academic Publishers, Dordrecht), Vol. 167.

Stribling, R., and S.L. Miller, 1986, "Energy yields for hydrogen cyanide and formaldehyde syntheses: The HCN and amino acid concentrations in the primitive ocean," Origins Life Evol. Biosphere 17, 261.

Stribling, R., and S.L. Miller, 1991, “Template-directed synthesis of oligonucleotides under eutectic conditions," J. Mol. Evol. 32, 289 .

Stroeve, J., M. M. Holland, W. Meier, T. Scambos, and M. Serreze, 2007, "Arctic sea ice decline: Faster than forecast," Geophys. Res. Lett. 34, L09501.

Style, R. W., and M. G. Worster, 2009, "Frost flower formation on sea ice and lake ice," Geophys. Res. Lett. 36, L11501.

Sugiyama, T., 1994, "Ion-recombination nucleation and growth of ice particles in noctilucent clouds," J. Geophys. Res. 99, 3915.

Suter, M.T., P.U. Andersson, and J.B.C. Pettersson, 2006, "Surface properties of water ice at 150-191 K studied by elastic helium scattering," J. Chem. Phys. 125, 174704.

Suter, M. T., P.U. Andersson, and J.B.C. Pettersson, 2007, "Formation of water-ammonia ice on graphite studied by elastic helium scattering," Chem. Phys. Lett. 445, 208.

Suzuki, S., A. Nakajima, N. Yoshida, M. Sakai, A. Hashimoto, Y. Kameshima, and K. Okada, 2007, "Freezing of water droplets on silicon surfaces coated with various silanes," Chem. Phys. Lett. 445, 37.

Svanberg, M., J.B. C. Pettersson, and K. Bolton, 2000, "Coupled $\mathrm{QM} / \mathrm{MM}$ molecular dynamics simulations of $\mathrm{HCl}$ interacting with ice surfaces and water clusters-evidence of rapid ionization," J. Phys. Chem. A 104, 5787.

Svensson, E. A., C. Delval, P. von Hessberg, M. S. Johnson, and J.B.C. Pettersson, 2009, "Freezing of water droplets colliding with kaolinite particles," Atmos. Chem. Phys. 9, 4295.

Swanson, B. D., M. J. Bacon, E. J. Davis, and M. B. Baker, 1999, "Electrodynamic trapping and manipulation of ice crystals," Q. J. R. Meteorol. Soc. 125, 1039.

Szyrmer, W., and I. Zawadzki, 1997, "Biogenic and anthropogenic sources of ice-forming nuclei: A review," Bull. Am. Meteorol. Soc. 78, 209.

Tabazadeh, A., 2005, "Organic aggregate formation in aerosols and its impact on the physicochemical properties of atmospheric particles," Atmos. Environ. 39, 5472.

Tabazadeh, A., Y.S. Djikaev, P. Hamill, and H.J. Reiss, 2002, "Laboratory evidence for surface nucleation of solid polar stratospheric cloud particles,” J. Phys. Chem. A 106, 10238. 
Tajima, Y., T. Matsuo, and H. Suga, 1984, "Calorimetric studies of phase transition in hexagonal ice doped with alkali hydroxides," J. Phys. Chem. Solids 45, 1135.

Takenaka, N., A. Ueda, and Y. Maeda, 1992, "Acceleration of the rate of nitrite oxidation by freezing in aqueous solution," Nature (London) 358, 736.

Tegler, S. C., D. A. Weintraub, T. W. Rettig, Y. J. Pendleton, D. C. B. Whittet, and C.A. Kulesa, 1995, "Evidence for chemical processing of precometary icy grains in circumstellar environments of pre-main-sequence stars," Astrophys. J. 439, 279.

Thayer, J.P., and W.L. Pan, 2006, "Lidar observations of sodium density depletions in the presence of polar mesospheric clouds," J. Atmos. Sol. Terr. Phys. 68, 85.

Thomas, D. N., and G. S. Dieckmann, 2010, Eds., Sea Ice (WileyBlackwell, Oxford, UK), 2nd ed.

Thomas, G.E., and J. Olivero, 2001, "Noctilucent clouds as possible indicators of global change in the mesosphere," Adv. Space Res. 28, 937.

Thomas, G. E., J.J. Olivero, M. DeLand, and E. P. Shettle, 2003, "Comment on "are noctilucent clouds truly a 'miner's canary' for global change?," Eos Trans. AGU 84, 352.

Thomas, G. E., J. J. Olivero, E. J. Jensen, W. Schroeder, and O. B. Toon, 1989, "Relation between increasing methane and the presence of ice clouds at the mesopause," Nature (London) 338, 490.

Thomas, P., J. Veverka, and S. Dermott, 1986, "Small Satellites," Satellites (University of Arizona Press, Tucson).

Thomas, R. D., et al., 2010, "Hot water from cold. The dissociative recombination of water cluster ions," J. Phys. Chem. A 114, 4843.

Thompson, D. W., and J.M. Wallace, 2000, "Annular modes in extratropical circulation, Part I: Month-to-Month Variability," J. Clim. 13, 1000.

Thomson, E. S., L. Benatov, and J. S. Wettlaufer, 2010, "Erratum: Abrupt grain boundary melting in ice," Phys. Rev. E 82, 039907 (E) [Phys. Rev. E 70, 061606 (2004)].

Thomson, E.S., X.R. Kong, P.U. Andersson, N. Markovic, and J. B.C. Pettersson, 2011, "Collision dynamics and solvation of water molecules in a liquid methanol film," J. Phys. Chem. Lett. 2, 2174.

Thomson, E. S., J. S. Wettlaufer, and L. A. Wilen, 2009, “A direct optical method for the study of grain boundary melting, Rev. Sci. Instrum. 80, 103903.

Thomson, W., 1871, "On the equilibrium of vapour at a curved surface of liquid," Philos. Mag. 42, 448.

Thorndike, A.S., D. A. Rothrock, G. A. Maykut, and R. Colony, 1975, “The thickness distribution of sea ice," J. Geophys. Res. 80, 4501.

Thorsteinsson, T., J. Kipfstuhl, and H. Miller, 1997, "Textures and fabrics in the grip ice core," J. Geophys. Res. 102, 26583.

Thrower, J.D., et al., 2010, "Photon- and electron-stimulated desorption from laboratory models of interstellar ice grains," J. Vac. Sci. Technol. A 28, 799.

Thrower, J.D., et al., 2008, "Surface science investigations of photoprocesses in model interstellar ices," J. Vac. Sci. Technol. A 26, 919.

Tiedje, T., K. A. Mitchell, B. Lau, A. Ballestad, and E. Nodwell, 2006, "Radiation transport model for ablation hollows on snowfields," J. Geophys. Res. 111, F02015.

Tielens, A.G.G.M., 2005, The Physics and Chemistry of the Interstellar Medium (Cambridge University Press, Cambridge, England).

Tielens, A. G. G. M., and W. Hagen, 1982, "Model calculations of the molecular composition of interstellar grain mantles,"
Astron. Astrophys. 114, 245 [http://adsabs.harvard.edu/full/ 1982A\&A...114..245T].

Tizek, H., Knözinger, E., and Grothe, H., 2002, “X-ray diffraction studies on nitric acid dihydrate," Phys. Chem. Chem. Phys. 4, 5128.

Tizek, H., H. Grothe, and E. Knözinger, 2004, "Gas-phase deposition of acetonitrile: an attempt to understand Ostwald's step rule on a molecular basis," Chem. Phys. Lett. 383, 129.

Tolbert, M. A., and O. B. Toon, 2001, "Atmospheric science. solving the PSC mystery," Science 292, 61.

Toon, O.B., and M.A. Tolbert, 1995, "Spectroscopic evidence against nitric acid trihydrate in polar stratospheric clouds," Nature (London) 375, 218.

Toubin, C., S. Picaud, P. N. M. Hoang, C. Girardet, B. Demirdjian, D. Ferry, and J. Suzanne, 2001, "Dynamics of ice layers deposited on $\mathrm{MgO}(001)$ : Quasielastic neutron scattering experiments and molecular dynamics simulations," J. Chem. Phys. 114, 6371.

Trainer, M. G., M. A. Tolbert, C. P. McKay, and O. B. Toon, 2010, "Enhanced $\mathrm{CO}_{2}$ trapping in water ice via atmospheric deposition with relevance to Mars," Icarus 206, 707.

Tribello, G., B. Slater, and C. G. Salzmann, 2006, "A blind structure prediction of ice XIV," J. Am. Chem. Soc. 128, 12594.

Trinks, H., W. Schröder, and C. K. Bierbricher, 2005, "Sea ice as a promoter of the emergence of first life," Origins of Life and Evolution of Biospheres 35, 429.

Trujillo, C. A., M.E. Brown, K. M. Barkume, E. L. Schaller, and D. L. Rabinowitz, 2007, "The surface of $2003 \mathrm{EL}_{61}$ in the nearinfrared," Astrophys. J. 655, 1172.

Tuhkuri, J., and M. Lensu, 2002, "Laboratory tests on ridging and rafting of ice sheets," J. Geophys. Res. 107, 3125.

Tulk, C. A., C. J. Benmore, J. Urquidi, D. D. Klug, J. Neuefeind, B. Tomberli, and P. A. Egelstaff, 2002, "Structural studies of several distinct metastable forms of amorphous ice," Science 297, 1320.

Ullerstam, M., T. Thornberry, and J. Abbatt, 2005, "Uptake of gasphase nitric acid to ice at low partial pressures: evidence for unsaturated surface coverage," Faraday Discuss. 130, 211.

Uras-Aytemiz, N., J. Sadlej, J. P. Devlin, and V. Buch, 2006, “HCl solvation at the surface and within methanol clusters/nanoparticles II: Evidence for molecular wires," J. Phys. Chem. B 110, 21751. VandeVondele, J., M. Krack, F. Mohamed, M. Parrinello, T. Chassaing, and J. Hutter, 2005, "Quickstep: Fast and accurate density functional calculations using a mixed Gaussian and plane waves approach," Comput. Phys. Commun. 167, 103.

van Dishoeck, E. F., 2004, "Iso spectroscopy of gas and dust: From molecular clouds to protoplanetary disks," Annu. Rev. Astron. Astrophys. 42, 119.

Vaughan, D. G., 2007, “The Antarctic ice sheet," in Glacier Science and Environmental Change, edited by P.G. Knight (Blackwell Publishing, Malden, MA), 1st ed., p. 209.

Vega, C., J. L. F. Abascal, M. M. Conde, and J. L. Aragones, 2009, "What ice can teach us about water interactions: a critical comparison of the performance of different water models," Farad. Disc. Soc. Chem. 141, 251.

Vehkamäki, H., 2006, Classical Nucleation Theory in Multicomponent Systems (Springer, Berlin).

Vella, D., and J.S. Wettlaufer, 2007, "Finger rafting: a generic instability of floating elastic sheets," Phys. Rev. Lett. 98, 088303.

Vella, D., and J.S. Wettlaufer, 2008, "Explaining the patterns formed by ice floe interaction," J. Geophys. Res. 113, C11011.

Venkatesh, C. G., S. A. Rice, and A. H. Narten, 1974, "Amorphous solid water: An X-ray diffraction study,” Science 186, 927. 
Vidali, G., J.E. Roser, G. Manicò, and V. Pirronello, 2004, "Experimental study of the formation of molecular hydrogen and carbon dioxide on dust grain analogues, Space Life Sciences: Steps toward Origin(s) of Life," Adv. Space Res. 33, 6.

Viti, S., M. P. Collings, J. W. Dever, M. R. S. McCoustra, and D. A. Williams, 2004, "Evaporation of ices near massive stars: models based on laboratory temperature programmed desorption data," Mon. Not. R. Astron. Soc. 354, 1141.

Vlassov, A. V., B. H. Johnston, L. F. Landweber, and S. A. Kazakov, 2004, "Ligation activity of fragmented ribozymes in frozen solution: implications for the RNA world," Nucleic Acids Res. 32, 2966

Voigt, C., et al., 2003, "In situ mountain-wave polar stratospheric cloud measurements: Implications for nitric acid trihydrate formation," J. Geophys. Res. 108, 8331.

Voigt, C., et al., 2005, "Nitric acid trihydrate (NAT) formation at low NAT supersaturation in polar stratospheric clouds (PSCs)," Atmos. Chem. Phys. 5, 1371.

Voigt, C., et al., 2000, "Nitric Acid Trihydrate (NAT) in Polar Stratospheric Clouds," Science 290, 1756.

von Blohn, N., S. K. Mitra, K. Diehl, and S. Borrmann, 2005, “The ice nucleating ability of pollen: Part iii: New laboratory studies in immersion and contact freezing modes including more pollen types," Atmos. Res. 78, 182.

von Hessberg, P., N. Pouvesle, A. K. Winkler, G. Schuster, and J. N. Crowley, 2008, "Interaction of formic and acetic acid with ice surfaces between 187 and $227 \mathrm{~K}$. Investigation of single speciesand competitive adsorption," Phys. Chem. Chem. Phys. 10, 2345.

Vonnegut, B., 1994, "The atmospheric electricity paradigm," Bull. Am. Meteorol. Soc. 75, 53.

von Zahn, U., 2003, “Are noctilucent clouds truly a "miner's canary" for global change?," Eos Trans. AGU 84, 261.

Wadhams, P., 1992, "Sea ice thickness distribution in the Greenland Sea and Eurasian Basin, May 1987," J. Geophys. Res. 97, 5331.

Wadhams, P., 2000, Ice in The Ocean (CRC Press, Boca Raton, FL).

Wadhams, P., and M. Doble, 2008, "Digital terrain mapping of the underside of sea ice from a small AUV," Geophys. Res. Lett. 35, L01501.

Walkington, I., M. A. Morales Maqueda, and A. J. Willmott, 2007, "A robust and computationally efficient model of a twodimensional coastal polynya," Ocean Modelling 17, 140.

Wang, J., J. Zhang, E. Watanabe, M. Ikeda, K. Mizobata, J. Walsh, X. Bai, and B. Wu, 2009, "Is the dipole anomaly a major driver to record lows in Arctic summer sea ice extent?," Geophys. Res. Lett. 36, L05706.

Wang, K., and C. Wang, 2009, "Modeling linear kinematic features in pack ice," J. Geophys. Res. 114, C12011.

Wang, M., and J.E. Overland, 2009, "A sea ice free summer Arctic within 30 years?," Geophys. Res. Lett. 36, L07502.

Wang, P.K., 2002, "Ice microdynamics," in Advances in Geophysics, edited by R. Dmowska and B. Saltzman (Academic Press, New York), Vol. 45.

Watanabe, M., and S. Arai, 1995, "Applications of bacterial ice nucleation activity in food processing," in Biological Ice Nucleation and Its Applications, edited by R. E. Lee, Jr., G. J. Warren, and L. V. Gusta (APS Press, St. Paul, MN), p. 299.

Watanabe, N., Y. Kimura, A. Kouchi, T. Chigai, T. Hama, and V. Pirronello, 2010, "Direct measurements of hydrogen atom diffusion and the spin temperature of nascent $\mathrm{H}_{2}$ molecule on amorphous solid water," Astrophys. J. Lett. 714, L233.
Watanabe, N., A. Nagaoka, H. Hidaka, T. Shiraki, T. Chigai, and A. Kouchi, 2006, "Dependence of the effective rate constants for the hydrogenation of $\mathrm{CO}$ on the temperature and composition of the surface," Planet Space Sci. 54, 1107.

Weeks, W.F., 2010, On Sea Ice (University of Alaska Press, Fairbanks).

Wei, X., P. B. Miranda, and Y.R. Shen, 2001, "Surface vibrational spectroscopic study of surface melting of ice," Phys. Rev. Lett. 86, 1554.

Weinheimer, A.J., and C.A. Knight, 1987, "Scheiner's halo: Cubic ice or polycrystalline hexagonal ice?," J. Atmos. Sci. 44, 3304.

Weiss, J., 2008, "Intermittency of principal stress directions within Arctic sea ice," Phys. Rev. E 77, 056106.

Wettlaufer, J.S., 1994, "Introduction to crystallization phenomena in natural and artificial sea ice," in Physics of Ice-Covered Seas, Lecture Notes from Savonlinna Summer School (University of Helsinki, Finland), Vol. 1, p. 105.

Whalley, E., 1983, "Cubic ice in nature," J. Phys. Chem. 87, 4174.

Wilchinsky, A. V., and D L. Feltham, 2006, "Anisotropic model for granular sea ice dynamics,” J. Mech. Phys. Solids 54, 1147.

Wilen, L. A., and J. G. Dash, 1995, "Giant facets at grain boundary grooves," Science 270, 1184.

Williams, D. A., W. A. Brown, S. D. Price, J. M. C. Rawlings, and S. Viti, 2007, "Molecules, ices and astronomy," Astron. Geophys. 48, 25.

Williams, D. A., and T. W. Hartquist, 1999, “The chemistry of starforming regions," Acc. Chem. Res. 32, 334.

Williams, D. A., and E. Herbst, 2002, “It's a dusty universe: surface science in space," Surf. Sci. 500, 823.

Winkel, K., D. T. Bowron, T. Loerting, E. Mayer, and J. L. Finney, 2009, "Relaxation effects in low density amorphous ice: Two distinct structural states observed by neutron diffraction," J. Chem. Phys. 130, 204502.

Winkel, K., M.S. Elsaesser, E. Mayer, and T. Loerting, 2008, "Water polyamorphism: Reversibility and (dis)continuity," J. Chem. Phys. 128, 044510.

Winkler, A. K., N.S. Holmes, and J.N. Crowley, 2002, "Interaction of methanol, acetone and formaldehyde with ice surfaces between 198 and 223 K," Phys. Chem. Chem. Phys. 4, 5270.

Wong, M. H., G. L. Bjoraker, M. D. Smith, F. M. Flasar, and C. A. Nixon, 2004, "Identification of the $10-\mu \mathrm{m}$ ammonia ice feature on Jupiter," Planet. Space Sci. 52, 385.

Zahnle, K. L., and J.C.G. Walker, 1982, "The evolution of solar ultraviolet luminosity," Rev. Geophys. 20, 280.

Zasowski, G., F. Kemper, D. M. Watson, E. Furlan, C. J. Bohac, C. Hull, and J.D. Green, 2009, "Spitzer infrared spectrograph observations of class I/II objects in Taurus: Composition and thermal history of the circumstellar ices," Astrophys. J. 694, 459.

Zhou, X.L., H. Beine, R. Honrath, J.D. Fuentes, W. Simpson, P. B. Shepson, and J.W. Bottenheim, 2001, "Snowpack photochemical production of HONO: a major source of $\mathrm{OH}$ in the Arctic boundary layer in springtime," Geophys. Res. Lett. 28, 4087.

Zobrist, B., C. Marcolli, D. A. Pedernera, and T. Koop, 2008, "Do atmospheric aerosols form glasses?," Atmos. Chem. Phys. 8, 5221.

Zondlo, M. A., P. K. Hudson, A. J. Prenni, and M. A. Tolbert, 2000, "Chemistry and microphysics of polar stratospheric clouds and cirrus clouds," Annu. Rev. Phys. Chem. 51, 473. 